\title{
Feature Extraction for the Differentiation of Dry and Wet Cough Sounds
}

by

Hanieh Chatrzarrin, B.Eng.

A thesis submitted to the Faculty of Graduate and Postdoctoral Affairs

in partial fulfillment of the requirements for the degree of

Master of Applied Science in Biomedical Engineering

(Ottawa -Carleton Institute for Biomedical Engineering (OCIBME))

Department of Systems and Computer Engineering

Carleton University

Ottawa, Ontario, Canada, K1S 5B6

September 2011

(C) Copyright 2011, Hanieh Chatrzarrin 
Library and Archives

Canada

Published Heritage

Branch

395 Wellington Street

Ottawa ON K1A ON4

Canada
Bibliothèque et

Archives Canada

Direction du

Patrimoine de l'édition

395 , rue Wellington

Ottawa ON K1A 0N4

Canada
Your file Votre référence
ISBN: $978-0-494-83057-4$
Our file Notre référence
ISBN: $978-0-494-83057-4$

\section{NOTICE:}

The author has granted a nonexclusive license allowing Library and Archives Canada to reproduce, publish, archive, preserve, conserve, communicate to the public by telecommunication or on the Internet, loan, distribute and sell theses worldwide, for commercial or noncommercial purposes, in microform, paper, electronic and/or any other formats.

The author retains copyright ownership and moral rights in this thesis. Neither the thesis nor substantial extracts from it may be printed or otherwise reproduced without the author's permission.
AVIS:

L'auteur a accordé une licence non exclusive permettant à la Bibliothèque et Archives Canada de reproduire, publier, archiver, sauvegarder, conserver, transmettre au public par télécommunication ou par l'Internet, prêter, distribuer et vendre des thèses partout dans le monde, à des fins commerciales ou autres, sur support microforme, papier, électronique et/ou autres formats.

L'auteur conserve la propriété du droit d'auteur et des droits moraux qui protège cette thèse. $\mathrm{Ni}$ la thèse ni des extraits substantiels de celle-ci ne doivent être imprimés ou autrement reproduits sans son autorisation.
In compliance with the Canadian Privacy Act some supporting forms may have been removed from this thesis.

While these forms may be included in the document page count, their removal does not represent any loss of content from the thesis.
Conformément à la loi canadienne sur la protection de la vie privée, quelques formulaires secondaires ont èté enlevés de cette thèse.

Bien que ces formulaires aient inclus dans la pagination, il n'y aura aucun contenu manquant.

\section{Canadä}


The undersigned recommend to

the Faculty of Graduate and Postdoctoral Affairs

acceptance of the thesis

Feature Extraction for the Differentiation of Dry and Wet Cough Sounds

\author{
submitted by \\ Hanieh Chatrzarrin, B.Eng. \\ in partial fulfillment of the requirements for \\ the degree of Master of Applied Science in Biomedical Engineering
}

Chair, Dr. Howard M. Schwartz, Department of Systems and Computer Engineering

Thesis Supervisor, Dr. Rafik Goubran

Carleton University

September 2011 


\section{Abstract}

Cough is one of the most common symptoms in all respiratory diseases. It is important to provide the healthcare professionals with useful clinical information such as frequency, severity and nature of cough to have a better diagnosis and hence better treatment. The main objective of this thesis is to analyze cough sounds and extract features that can differentiate dry and wet cough sounds. This thesis proposes two features to achieve this goal. The first feature is the number of peaks of the energy envelope of the cough signal. The second feature is the power ratio of two frequency bands of the second phase of the cough signal. A set of nine highly dry and eight highly wet cough recordings were used in this thesis. Using these two features, a clear separation was observed among the dry and wet cough recordings. Furthermore, a Graphical User Interface (GUI) was designed in this thesis as a tool to analyze the cough signals in both time and frequency domain. 


\section{Acknowledgments}

I would like to extend my sincere thanks to my supervisor Dr. Rafik Goubran for his constant guidance, encouragement, and mentorship throughout my thesis. I would also like to acknowledge the financial support from Natural Sciences and Engineering Research Council (NSERC), Healthcare Support through Information Technology Enhancements (hSITE) and Carleton University.

I would like to thank Dr. Vilas Joshi for his continuous support, guidance, sharing his knowledge and friendship throughout my journey at Carleton University. Also, I would like to thank Dr. Amaya Arcelus for her technical guidance and support.

I would like to thank my brother Alireza Chatrzarrin and also Peyman Moradshahi for their love and support.

Special thanks to my wonderful and loving parents, Ahmad Chatrzarrin and Farzaneh Rashidi, for their endless love and support throughout my life.

In the end, I would like to thank my husband, classmate, colleague and best friend Payam Moradshahi for his love, patience, constant encouragement and always being there for me and believing in me. 


\section{Table of Contents}

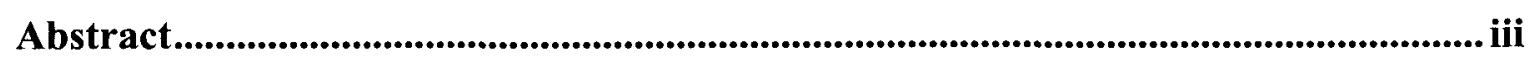

Acknowledgments ......................................................................................................................... iv

Table of Contents ................................................................................................................................. v

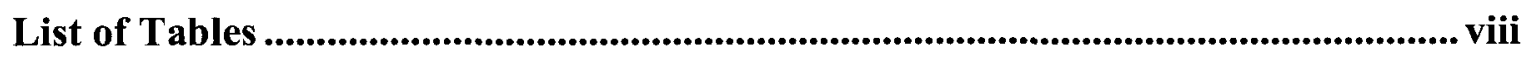

List of Figures....................................................................................................................... $\mathrm{x}$

List of Acronyms .................................................................................................. xvi

List of Symbols ............................................................................................................................... xviii

Chapter 1: Introduction ................................................................................................................ 1

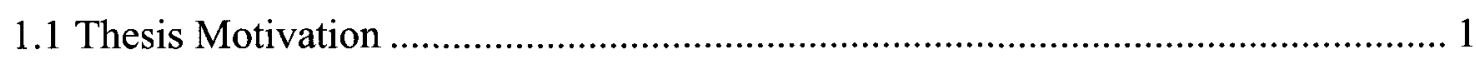

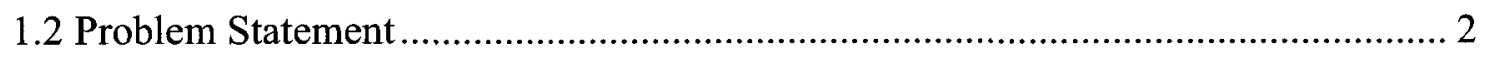

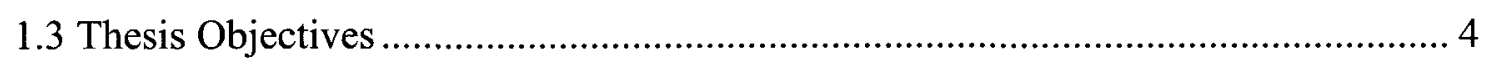

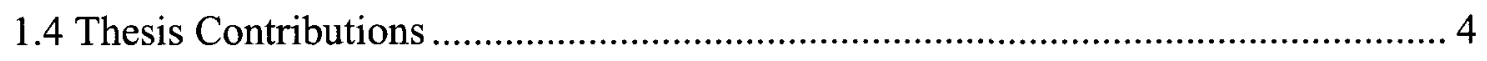

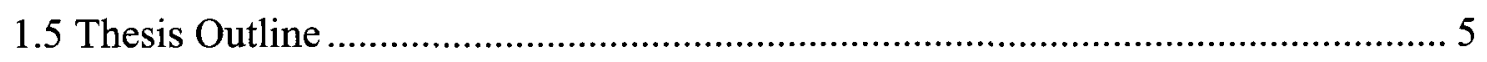

Chapter 2: Background Review ............................................................................................................ 7

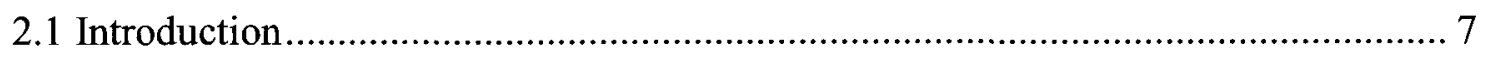

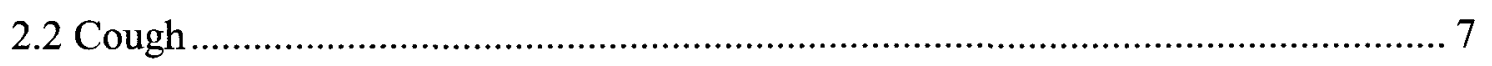

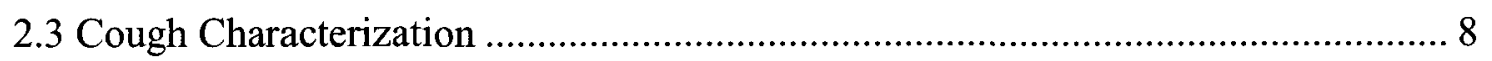




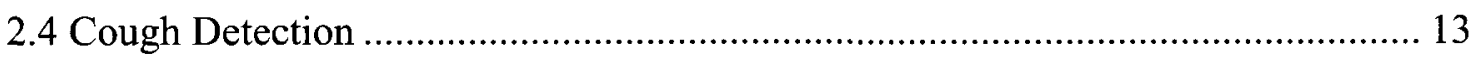

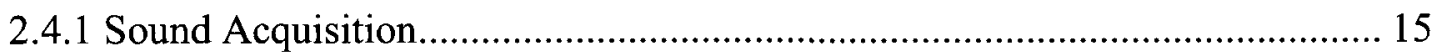

2.4.2 Feature Extraction Algorithm and Classification Techniques ......................... 17

2.5 Smart Home Technology .................................................................................... 20

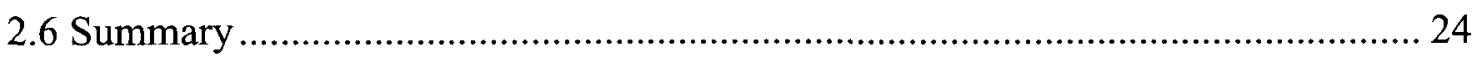

Chapter 3: Cough Sound Characteristics.......................................................................... 26

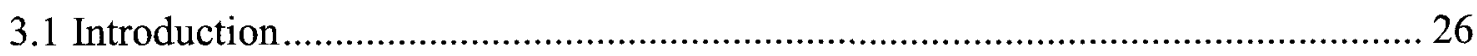

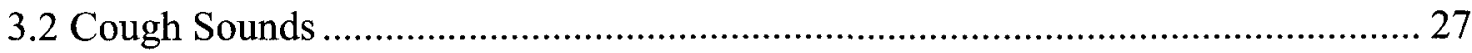

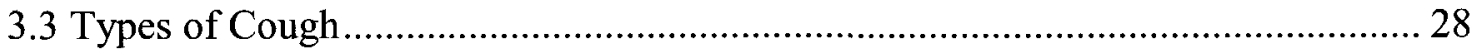

3.4 Common Characteristics of Cough Signal........................................................ 29

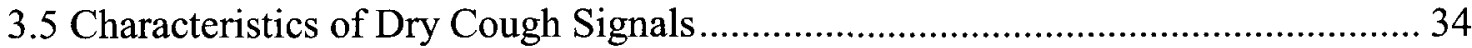

3.6 Characteristics of Wet Cough Signals .............................................................. 35

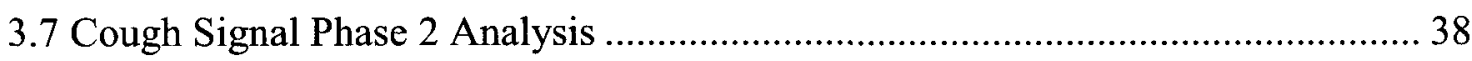

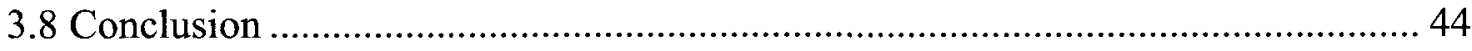

Chapter 4: Data Acquisition and Pre-Processing Algorithm ............................................ 45

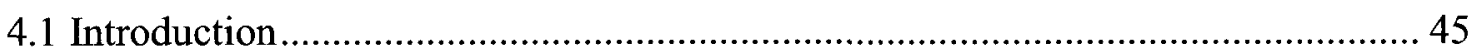

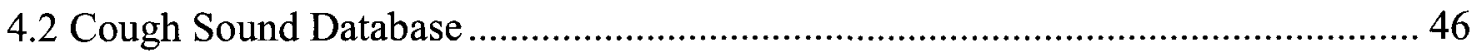

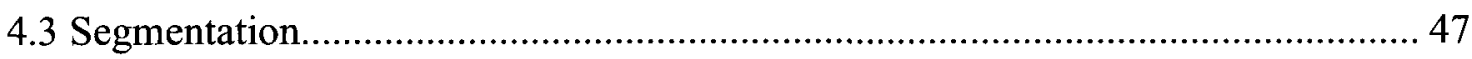

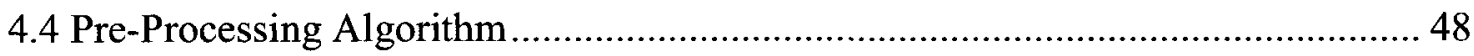

Chapter 5: Feature Extraction Algorithm...............................................................5 50

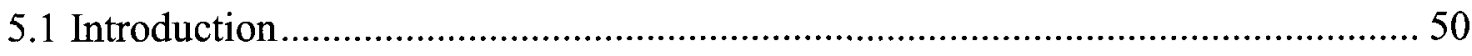

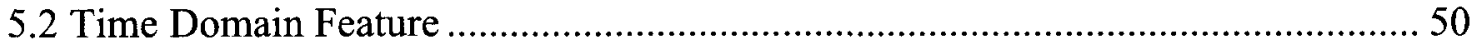

5.3 Energy Envelope Variation Detection .......................................................... 51

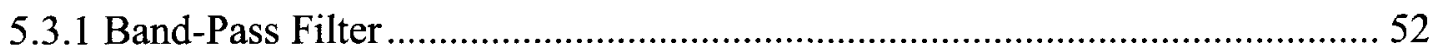

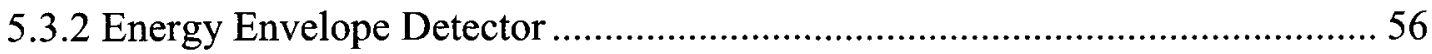




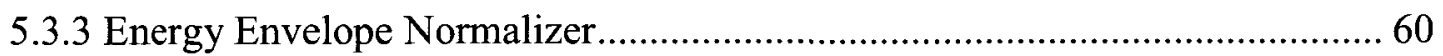

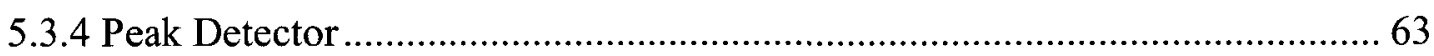

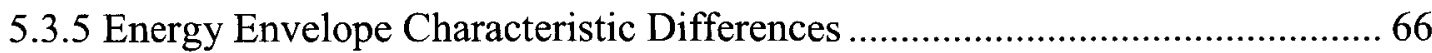

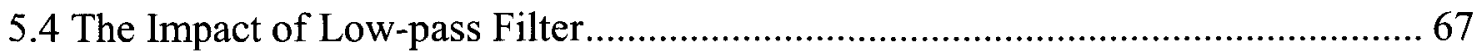

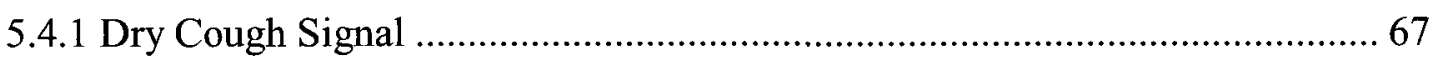

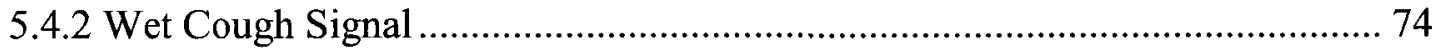

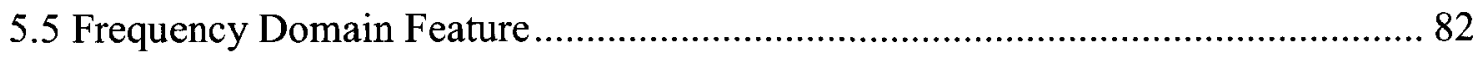

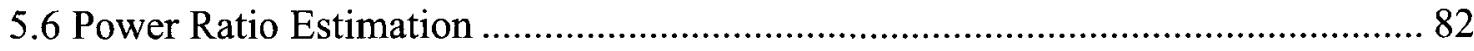

5.6.1 Cough Phase Detector .................................................................................. 83

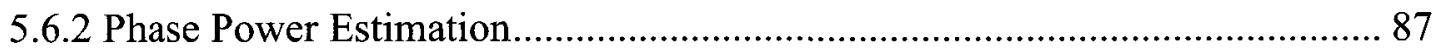

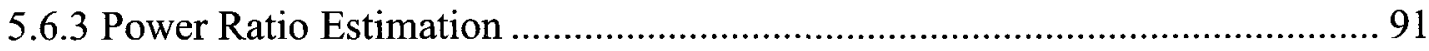

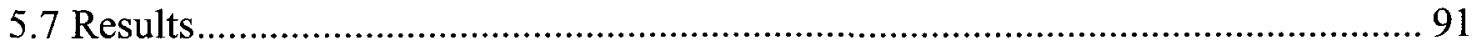

5.7.1 Results of Feature 1 Extraction Algorithm using Band-Pass Filter (BPF) ...... 91

5.7.2 Results of Feature 1 Extraction Algorithm using Low-Pass Filter (LPF) ....... 98

5.7.3 Results of Feature 2 Extraction Algorithm ................................................... 105

5.7.4 Results of Feature 1 vs. Feature 2 …………............................................ 106

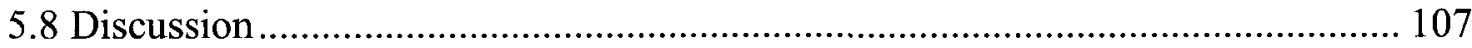

Chapter 6: Graphical User Interface ...................................................................................... 110

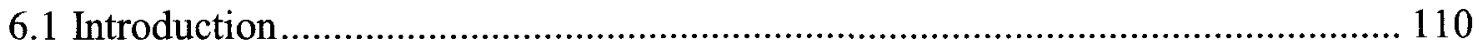

6.2 Graphical User Interface Design.................................................................... 110

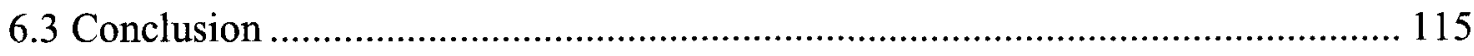

Chapter 7: Conclusion ......................................................................................................... 116

7.1 Thesis Conclusions and Contributions................................................................. 116

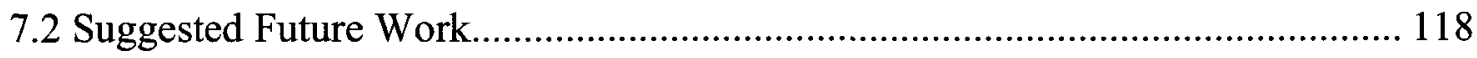

References ................................................................................................................................. 119 


\section{List of Tables}

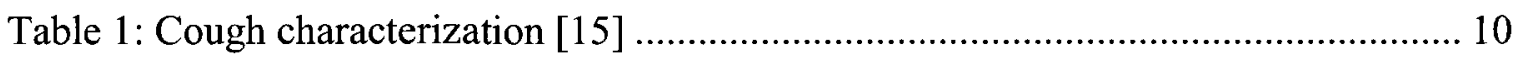

Table 2: Cough monitoring sensors [25] ……………......................................... 14

Table 3: Perquisite requirements of health monitoring at home [41] .............................. 23

Table 4: Acoustic properties of cough in healthy females and males [68] ...................... 32

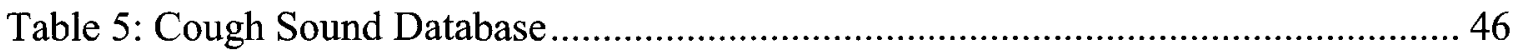

Table 6: Number of peaks of dry cough samples for frequency bands between $0-500 \mathrm{~Hz}$

92

Table 7: Number of peaks of dry cough samples for frequency bands between 550-1000

$\mathrm{Hz}$

Table 8: Number of peaks of wet cough samples for frequency bands between $0-500 \mathrm{~Hz}$ 94

Table 9: Number of peaks of wet cough samples for frequency bands between 550-1000

$\mathrm{Hz}$

Table 10: Number of peaks of dry cough samples using LPF with cut-off frequency

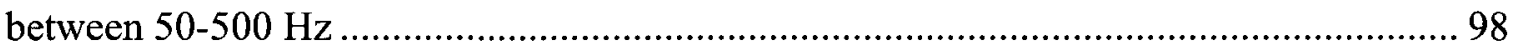


Table 11: Number of peaks of dry cough samples using LPF with cut-off frequency between $550-1000 \mathrm{~Hz}$ 99

Table 12: Number of peaks of wet cough samples using LPF with cut-off frequency between $50-500 \mathrm{~Hz}$ 100

Table 13: Number of peaks of wet cough samples using LPF with cut-off frequency between $550-1000 \mathrm{~Hz}$ 101

Table 14: $R p$ for both dry and wet cough samples. 105 


\section{List of Figures}

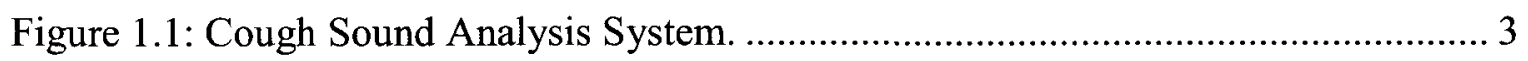

Figure 2.1: Sound Detection Algorithm................................................................... 14

Figure 2.2: Sound acquisition device installed on a) a portable device, b,c) bed headboards, d) underneath the bed, e) embedded in bed mat, and f) the ceiling............. 16

Figure 3.1: Throat anatomy including vocal cord and epiglottis [62] ............................ 28

Figure 3.2: Three-phase cough (first pattern) (Taken with permission from [25]). ......... 29

Figure 3.3: Two-phase cough (second pattern) Taken with permission from [25])........ 30

Figure 3.4: Peal cough (third pattern) (Taken with permission from [25])..................... 31

Figure 3.5: a) Dry cough signal with 3 phases: Phase 1, Phase 2, and Phase 3, b)

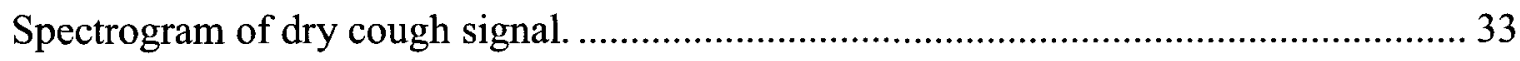

Figure 3.6: Dry cough signal along with its sound spectrogram a) Sample 1, b) Sample 2,

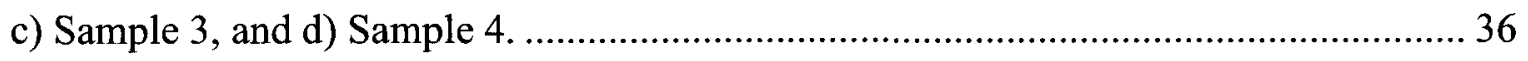

Figure 3.7: a) Wet cough signal with 3 phases: Phase 1, Phase 2, and Phase 3, b)

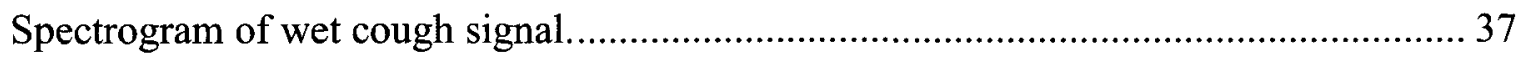


Figure 3.8: Wet cough signal along with its sound spectrogram a) Sample 1, b) Sample 2,

c) Sample 3, and d) Sample 4 . 39

Figure 3.9: Magnitude response of a low-pass filter with cut-off frequency $500 \mathrm{~Hz} . \ldots . . .40$

Figure 3.10: a) Dry cough sample 1, b) Low-pass filtered signal of dry cough sample 1, c) Dry cough sample 2, and d) Low-pass filtered signal of dry cough sample 2.

Figure 3.11: a) Wet cough sample 1, b) Low-pass filtered signal of wet cough sample 1,

c) Wet cough sample 2, and d) Low-pass filtered signal of wet cough sample 2. 43

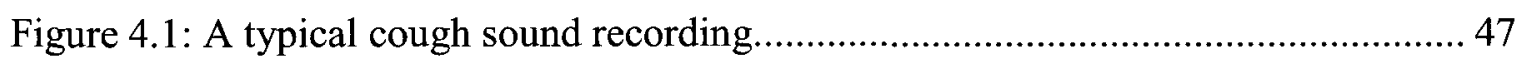

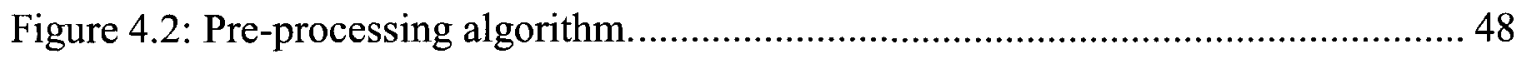

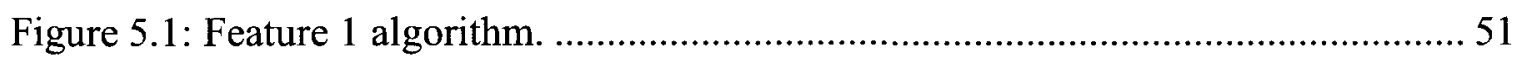

Figure 5.2: Magnitude response of a band-pass filter with cut-off frequencies of a) 200 and $250 \mathrm{~Hz}$. b) 250 and $300 \mathrm{~Hz}$. 52

Figure 5.3: a) Dry cough signal. b) Band-pass filtered dry cough signal at 200-250 Hz. c) Band-pass filtered dry cough signal at 250-300 Hz................................................ 54 Figure 5.4: a) Wet cough signal. b) Band-pass filtered wet cough signal at 200-250 Hz. c) Band-pass filtered wet cough signal at $250-300 \mathrm{~Hz}$. 55

Figure 5.5: Signal square of a band-pass filtered dry cough signal at a) $200-250 \mathrm{~Hz}, \mathrm{~b}$ ) $250-300 \mathrm{~Hz}$ 56

Figure 5.6: Signal square of a band-pass filtered wet cough signal at a) $200-250 \mathrm{~Hz}, \mathrm{~b}$ ) $250-300 \mathrm{~Hz}$ 57 
Figure 5.7: Magnitude response of a low-pass filter with cut-off frequency of $10 \mathrm{~Hz} . . . .58$

Figure 5.8: Energy envelope of dry cough signal at a) $200-250 \mathrm{~Hz}$ b) $250-300 \mathrm{~Hz}$....... 59

Figure 5.9: Energy envelope of wet cough signal at a) $200-250 \mathrm{~Hz}$ b) $250-300 \mathrm{~Hz}$...... 60

Figure 5.10: Normalized energy envelope of a dry cough signal at a) $200-250 \mathrm{~Hz}$, b) 250 $300 \mathrm{~Hz}$ 62

Figure 5.11: Normalized energy envelope of a wet cough signal at a) $200-250 \mathrm{~Hz}$, b) 250 $300 \mathrm{~Hz}$ 63

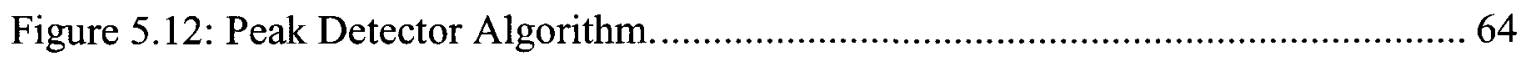

Figure 5.13: Normalized energy envelope and peaks of a) Dry cough sample 1, b) Dry cough sample 2, c) Wet cough sample 1 , and d) Wet cough sample 2. 65

Figure 5.14: a) Energy envelope of a dry cough, and b) Energy envelope of a wet cough. 66

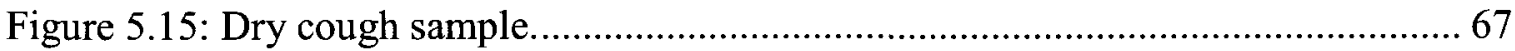

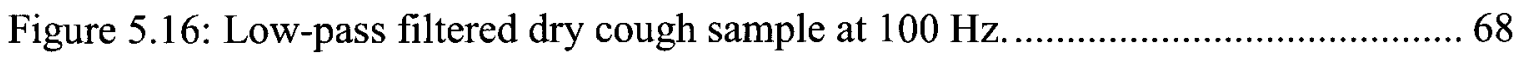

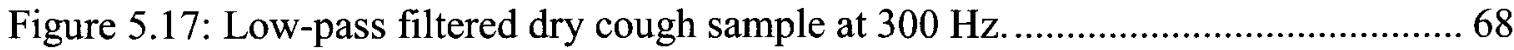

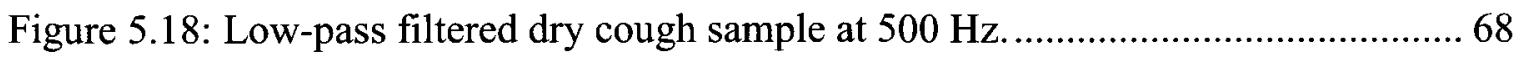

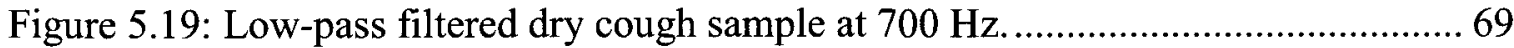

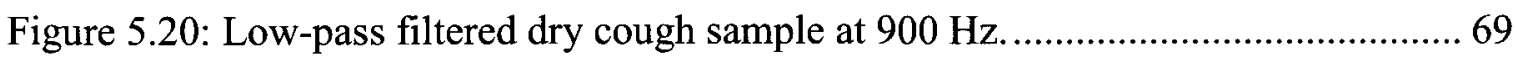

Figure 5.21: Signal squared of a low-pass filtered dry cough sound at $100 \mathrm{~Hz}$........... 70

Figure 5.22: Signal squared of a low-pass filtered dry cough sound at $300 \mathrm{~Hz}$........... 70 
Figure 5.23: Signal squared of a low-pass filtered dry cough sound at $500 \mathrm{~Hz}$. 70

Figure 5.24: Signal squared of a low-pass filtered dry cough sound at $700 \mathrm{~Hz}$. 71

Figure 5.25: Signal squared of a low-pass filtered dry cough sound at $900 \mathrm{~Hz}$ 71

Figure 5.26: Normalized energy envelope and peaks of low-pass filtered dry cough at $100 \mathrm{~Hz}$ 72

Figure 5.27: Normalized energy envelope and peaks of low-pass filtered dry cough at $300 \mathrm{~Hz}$. 72

Figure 5.28: Normalized energy envelope and peaks of low-pass filtered dry cough at $500 \mathrm{~Hz}$ 73

Figure 5.29: Normalized energy envelope and peaks of low-pass filtered dry cough at $700 \mathrm{~Hz}$ 73

Figure 5.30: Normalized energy envelope and peaks of low-pass filtered dry cough at $900 \mathrm{~Hz}$ 74

Figure 5.31: Wet cough sample. 75

Figure 5.32: Low-pass filtered wet cough sample at $100 \mathrm{~Hz}$. 75

Figure 5.33: Low-pass filtered wet cough sample at $300 \mathrm{~Hz}$. 76

Figure 5.34: Low-pass filtered wet cough sample at $500 \mathrm{~Hz}$. 76

Figure 5.35: Low-pass filtered wet cough sample at $700 \mathrm{~Hz}$. 76

Figure 5.36: Low-pass filtered wet cough sample at $900 \mathrm{~Hz}$ 77

Figure 5.37: Signal squared of a low-pass filtered wet cough sound at $100 \mathrm{~Hz}$ 77 
Figure 5.38: Signal squared of a low-pass filtered wet cough sound at $300 \mathrm{~Hz} \ldots \ldots \ldots \ldots \ldots . . .78$

Figure 5.39: Signal squared of a low-pass filtered wet cough sound at $500 \mathrm{~Hz}$.............. 78

Figure 5.40: Signal squared of a low-pass filtered wet cough sound at $700 \mathrm{~Hz} \ldots \ldots \ldots \ldots \ldots . . .78$

Figure 5.41: Signal squared of a low-pass filtered wet cough sound at $900 \mathrm{~Hz} \ldots \ldots \ldots \ldots \ldots . . .79$

Figure 5.42: Normalized energy envelope and peaks of low-pass filtered wet cough at $100 \mathrm{~Hz}$

Figure 5.43: Normalized energy envelope and peaks of low-pass filtered wet cough at $300 \mathrm{~Hz}$ 80

Figure 5.44: Normalized energy envelope and peaks of low-pass filtered wet cough at $500 \mathrm{~Hz}$ 80

Figure 5.45: Normalized energy envelope and peaks of low-pass filtered wet cough at $700 \mathrm{~Hz}$ 81

Figure 5.46: Normalized energy envelope and peaks of low-pass filtered wet cough at $900 \mathrm{~Hz}$ 81

Figure 5.47: Power ratio estimation feature extraction algorithm. 82

Figure 5.48: Cough sound divided into 4 equal parts. 83

Figure 5.49: a) Dry cough signal, b) Phase 1, c) Phase 2, and d) Phase 3...................... 85

Figure 5.50: a) Wet cough signal, b) Phase 1, c) Phase 2, and d) Phase 3..................... 86

Figure 5.51: Power of a dry cough a) Phase 1, b) Phase 2, and c) Phase 3..................... 87

Figure 5.52: Power of a wet cough a) Phase 1, b) Phase 2, and c) Phase 3..................... 88 
Figure 5.53: $P 2_{\text {norm }}(\mathrm{f})$ of a) Dry sample $\left.1, \mathrm{~b}\right)$ Dry sample 2, c) Dry sample 3, d) Wet sample 1, e) Wet sample2, and d) Wet sample 3....................................................... 90

Figure 5.54: Number of peaks at $150-200 \mathrm{~Hz}$ for both dry and wet cough signals......... 96

Figure 5.55: Number of peaks at $200-250 \mathrm{~Hz}$ for both dry and wet cough signals......... 96

Figure 5.56: Number of peaks at $250-300 \mathrm{~Hz}$ for both dry and wet cough signals......... 97

Figure 5.57: Number of peaks at 150-200 Hz for both dry and wet cough signals....... 102

Figure 5.58: Number of peaks at 200-250 Hz for both dry and wet cough signals....... 102

Figure 5.59: Number of peaks at 250-300 Hz for both dry and wet cough signals....... 103

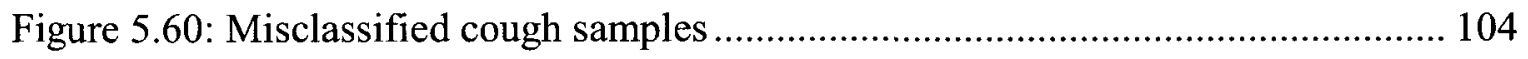

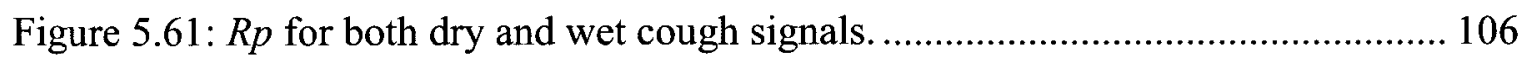

Figure 5.62: Separation of nine highly dry and eight highly wet cough sounds using

Feature 1 and Feature 2

Figure 5.63: Separation of dry and wet cough samples using Feature 1 and Feature 2.. 109

Figure 6.1: Graphical User Interface snapshot. ......................................................... 111

Figure 6.2: Show the STFT of the input signal in a separate figure. ............................. 112

Figure 6.3: Functionality of the Feature 1 panel.......................................................... 113

Figure 6.4: Functionality of the Feature 2 panel......................................................... 114 


\section{List of Acronyms}

$\begin{array}{ll}\text { AI } & \text { Artificial Intelligence } \\ \text { ANN } & \text { Artificial Neural Network } \\ \text { BPF } & \text { Band-Pass Filter } \\ \text { COPD } & \text { Chronic Obstructive Pulmonary Disease } \\ \text { CPFs } & \text { Cough Peak Flows } \\ \text { DSP } & \text { Digital Signal Processing } \\ \text { ECC } & \text { Energy Cepstral Coefficient } \\ \text { FFT } & \text { Fast Fourier Transform } \\ \text { GUI } & \text { Graphical User Interface } \\ \text { HMM } & \text { Hidden Markov Model } \\ \text { LPC } & \text { Linear Predictive Coding }\end{array}$

xvi 


$\begin{array}{ll}\text { LPF } & \text { Low-Pass Filter } \\ \text { MFCC } & \text { Mel-Frequency Cepstral Coefficient } \\ \text { PDA } & \text { Personal Digital Assistance } \\ \text { PSD } & \text { Power Spectral Density } \\ \text { RPM } & \text { Remote Patient Monitoring } \\ \text { STFT } & \text { Short-Time Fourier Transform } \\ \text { SNR } & \text { Signal-to-Noise Ratio } \\ \text { WHO } & \text { World Health Organization }\end{array}$




\section{List of Symbols}

$\begin{array}{ll}\mathrm{dB} & \text { Decibel } \\ \mathrm{E}(\mathrm{t}) & \text { Energy envelope in time domain } \\ \mathrm{Fs} & \text { Sampling frequency } \\ \mathrm{i} & \text { Power of Phase } 1 \text { of cough signal } \\ \mathrm{P} 1 & \text { Power of Phase } 2 \text { of cough signal } \\ \mathrm{P} 2 & \text { Normalized power of Phase } 2 \text { of cough signal } \\ \mathrm{P} 2 & \text { Power ratio } \\ \mathrm{Rp} & \text { Signal in time domain } \\ \mathrm{S}(\mathrm{t}) & \text { Square of signal } \\ \mathrm{S}^{2}(\mathrm{t}) & \text { Signal at index } \mathrm{i} \\ \mathrm{x}(\mathrm{i}) & \end{array}$

xviii 


\section{Chapter 1:}

\section{Introduction}

\subsection{Thesis Motivation}

Cough is one of the most common symptoms in all respiratory diseases. It is important to provide healthcare professionals with useful clinical information such as frequency, severity and nature of cough to have a better diagnosis and hence better treatment [1][2]. Describing the frequency, severity and nature of the cough is difficult for patients or their caregivers and in some cases impossible due to factors such as age, illness and lack of medical knowledge. For instance, patients who suffer from a cognitive impairment such as Alzheimer's disease may have difficulty remembering and/or describing their cough to their doctors. In addition, remembering the severity, frequency or nature of cough during sleep is challenging for patients of all ages.

Due to the challenges mentioned above, an automated cough monitoring system capable of determining the frequency, severity and nature of cough is highly desirable. This automated system could be integrated in smart homes or embedded in smart phones such as a blackberry device. The primary idea of smart homes is to monitor a patient's 
various health conditions remotely and continuously [3][4]. This includes monitoring biological sounds via microphones, monitoring the patient's condition at rest using pressure sensor mats, monitoring the force applied over time using smart grab bars, and much more [5][6]. Integrating cough information such as frequency, severity and nature along with other information collected by various smart home sensors could significantly increase the quality of information provided to healthcare professionals. This could improve the accuracy of diagnosis and treatment for patients [7][8].

\subsection{Problem Statement}

Characterizing coughs is an important factor in the diagnosis of respiratory diseases. Having information such as frequency, severity and nature of coughs can improve the treatment for respiratory diseases [9]. Cough could be classified as dry, wet, whooping and much more. Therefore, cough sound analysis has gained a lot of attention in the research community and in health care organizations.

Cough sound analysis consists of two main parts: cough sound detection and cough sound classification, as depicted in Figure 1.1. The cough sound detection portion involves collecting various sound types using microphones and extracting unique features specific to each type of sound collected (such as speech, laughter, snore, cough) and performing cough detection based on the extracted features. 
The cough sound classification portion, on the other hand, involves extracting unique features specific to each type of cough and classifying them into different types, such as dry or wet.

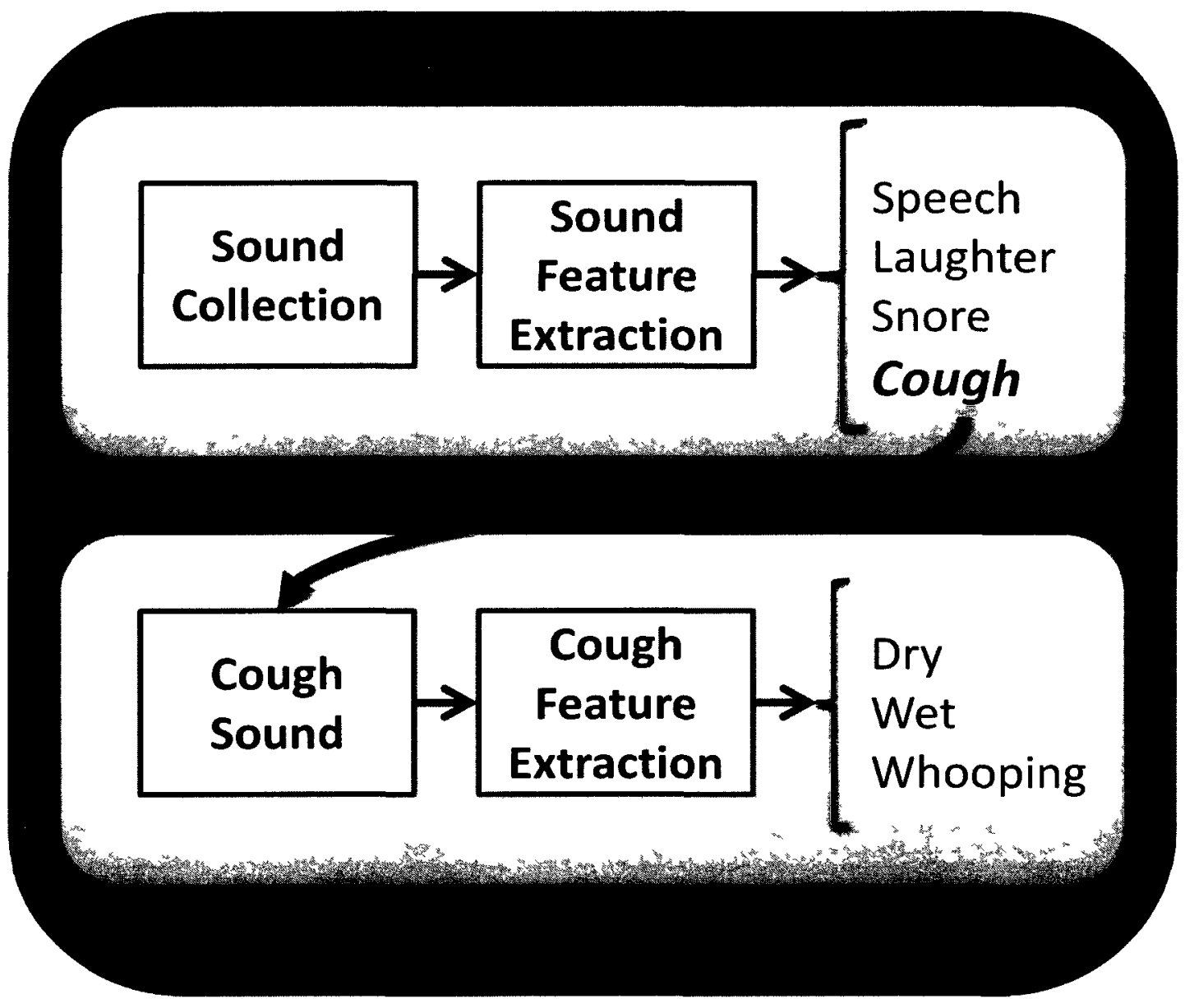

Figure 1.1: Cough Sound Analysis System.

The main focus of this thesis is to identıfy features that can differentiate dry and wet coughs. Based on these unique extracted features, the cough sounds could be classified into two types of cough, dry or wet. 


\subsection{Thesis Objectives}

The main objective of this thesis is to differentiate between dry and wet cough. A novel Digital Signal Processing (DSP) algorithm was designed in order to extract useful clinical information from cough sounds. Two feature extraction algorithms are proposed in order to differentiate cough signals into two classes, dry and wet. Cough signals were analyzed in both time and frequency domain. Features that could be used to discriminate the two types of cough were extracted. The feature extraction algorithms were verified with highly dry and highly wet cough sounds. Furthermore, a Graphical User Interface (GUI) was designed with various functionalities, as a tool to analyze cough sounds.

\subsection{Thesis Contributions}

The contributions that were made during this thesis research are listed below, followed by a detailed explanation of all the contributions in the following chapters of this thesis.

Contribution 1: Collected cough sound data and built a database of dry and wet cough sounds. The nature of the coughs was verified by clinicians.

Contribution 2: Analyzed cough signals and identified unique features associated with dry and wet coughs. 
Contribution 3: Proposed and implemented two feature extraction algorithms capable of differentiating dry and wet coughs. The first feature extraction algorithm is based on the number of peaks of the energy envelope of the cough signal. The second feature algorithm is based on the power ratio of the two frequency bands of the second phase of the cough signal. These features were tested using the collected cough sound database. This contribution was published in [10].

H. Chatrzarrin, A. Arcelus, R. Goubran, and F. Knoefel, "Feature extraction for the differentiation of dry and wet cough sounds," in Proc. IEEE International Symposium on Medical Measurements and Applications, Bari, Italy, May 2011, pp. 162-166.

Contribution 4: Designed a GUI in Matlab in order to analyze cough signals in time and frequency domains. The GUI has various functionalities such as computing the Fast Fourier Transform (FFT), Short-time Fourier Transform (STFT), signal energy and signal length of an input signal, in addition to playing the input signal and extracting the two features.

\subsection{Thesis Outline}

Chapter 2 will provide a detailed discussion and literature review on cough sound discrimination, cough sound detection and feature extraction algorithms. This chapter 
continues to describe the use of such cough analysis systems in smart homes and Remote Patient Monitoring systems (RPM).

Chapter 3 discusses the characteristics of dry and wet cough signals in both time domain and frequency domain. It will point out the similarities and differences between the two types of coughs.

Chapter 4 explains the cough sound database, cough sound acquisition and the preprocessing algorithm performed on the cough samples.

Chapter 5 explains the two feature extraction algorithms in detail. The first feature extraction algorithm computes the number of peaks of the energy envelope of the cough signals and the second feature extraction algorithm extracts the power ratio of the two frequency bands of the second phase of the cough signals. This chapter proceeds to show the result of the feature extraction algorithms and at the end discusses the results of the combination of the two features.

Chapter 6 explains the GUI and its various functionalities, followed by Chapter 7 , which presents the thesis conclusion and suggestions for future work. 


\section{Chapter 2:}

\section{Background Review}

\subsection{Introduction}

This chapter provides detailed discussions and literature review of cough sound analysis, smart home and Remote Patient Monitoring. Section 2.2 gives an introduction on the nature of cough. Section 2.3 describes cough sound discrimination. Cough sound detection, which consists of cough sound acquisition and feature extraction, is discussed in Section 2.4. Section 2.5 introduces smart homes and Remote Patient Monitoring systems. In the end, Section 2.6 gives a summary of this chapter.

\subsection{Cough}

Cough is the most common symptom of respiratory diseases. Fontana describes cough as "a three-phase expulsive motor act characterized by an aspiratory effort (aspiratory phase) followed by a forced expiratory effort initially against a closed glottis (compressive phase), and then by active glottal opening and rapid expiratory flow 
(expulsive phase). "[11]. The three phases mentioned by Fontana are referred to as Phase 1, Phase 2 and Phase 3 throughout this thesis.

Chronic respiratory diseases need constant care and it is required to monitor cough during day and night. The variables that can be measured from cough are respiratory flow and volume, respiratory muscle activity, respiratory pressures and cough sound. From the measured variables, cough frequency, severity and nature can be determined and that information can be provided to healthcare professionals for diagnostic and treatment purposes. Depending on the type of required information, any of the above variables could be measured. A combination of various measured variables could be more useful than individual variables. For instance, measuring only the cough frequency is not sufficient for differentiating between different types of coughs and it will not provide the healthcare professionals with enough information regarding any changes in coughs [11].

Frequently, patients cannot describe their coughs to their healthcare professionals. On the other hand, determining the nature of the cough might not be an obvious task for the healthcare professionals. Therefore, cough sound discrimination is desirable since it could assist healthcare professionals in determining the nature of cough and improve the accuracy of diagnosis and treatment.

\subsection{Cough Characterization}

This section introduces research that has focused on cough analysis and discrimination between different types of coughs. As mentioned previously, it is important to provide 
healthcare professionals with important cough related clinical information such as cough frequency, severity and nature for a better diagnosis and treatment [12][13][14].

There are various ways to discriminate cough based on either physiological mechanisms or pathological causes, as summarized in Table 1 [15]. In this thesis, the pathological discrimination is of interest.

Korpas et al. analyzed cough signal characteristics to extract clinical information such as the structural nature of the tissue during therapy and also the behaviour of the glottis [16]. Providing healthcare professionals with this information could have significant value in prognosis.

Another study focused on discriminating between productive (wet) and nonproductive (dry) coughs. Murata et al. compared the sound spectrogram and timeexpanded waveform of the normal cough with both productive and non-productive cough sounds. They found that there were no significant differences in duration of Phase 1 and 3 between the two groups; however, Phase 2 tended to have a longer duration in productive coughs [17]. 
Table 1: Cough characterization [15]

\begin{tabular}{|c|c|c|}
\hline Classificteaton & Deginnition: & Trangole \\
\hline Belhavioural & $\begin{array}{l}\text { Depends on the manner or } \\
\text { situation in which cough occurs }\end{array}$ & $\begin{array}{l}\text { Throat clearing, caused by } \\
\text { dusts and irritations; Voluntary } \\
\text { cough, mainly at night when } \\
\text { lying down }\end{array}$ \\
\hline Pattorology & $\begin{array}{l}\text { Evaluation of cough for the } \\
\text { purpose of medical diagnosis }\end{array}$ & Dry, wet, whooping, hacking, \\
\hline IDoration & $\begin{array}{l}\text { Evaluation of cough based on } \\
\text { its overall length }\end{array}$ & Acute, subacute, chronic \\
\hline Ieffect & $\begin{array}{l}\text { Evaluation of cough based on } \\
\text { its effect on an individual's } \\
\text { health condition }\end{array}$ & $\begin{array}{l}\text { Pain/distress, urinary } \\
\text { incontinence, rib fracture, } \\
\text { sleeplessness }\end{array}$ \\
\hline Gpade & $\begin{array}{l}\text { Evaluation of cough based on } \\
\text { its sensitivity }\end{array}$ & $\begin{array}{l}\text { Normal (Eutussia), sensitized } \\
\text { (Hypertussia), desensitized } \\
\text { (Hypotussia), pathological } \\
\text { (Dystussia), absent (Atussia) }\end{array}$ \\
\hline
\end{tabular}

Piirila et al. investigated the similarities of various types of pulmonary diseases in acoustics and dynamic features of cough. They collected cough samples from 31 patients with different types of respiratory diseases, such as asthma, acute and chronic bronchitis, 
tracheobronchial collapse and pulmonary fibrosis. They compared the flow dynamic and sound spectra of each group and found significant differences between the different groups. For instance, they discovered that the cough produced by asthma patients had a lower peak expiratory flow, longer duration of the first cough sound and lower frequency components as compared to the other types of coughs [18].

Another study investigated the mucus influence on cough sound generation. Hashimoto et al. analyzed the differences between dry and wet cough signals by computing the duration and root mean square of each phase of cough signal and also measuring rheological properties of the airway mucus. They found that Phase 2 had a longer duration in wet cough sounds. This finding shows that patients prolong Phase 2 of a cough for an effective expectoration of airway mucus [19].

Smith et al. examined how healthcare professionals describe coughs and whether they are able to diagnose a pulmonary disease condition. They randomly selected nine recorded coughs from patients with various respiratory diseases, such as asthma, idiopathic pulmonary fibrosis, laryngitis, and bronchiectasis. They categorised the coughs into 4 groups: (1) cough alone, (2) cough with mucus, (3) cough with wheeze, and (4) cough with wheeze and mucus. They performed an acoustic analysis of cough signals and identified that there were three distinct phases in a cough. Additionally, they discovered that cough with mucus had a significantly longer second phase and longer cough length than the other groups. Furthermore, they asked the healthcare professionals to indicate the 
patient gender, the most appropriate cough descriptor and a diagnosis by listening to the coughs. The healthcare professionals were able to discriminate between coughs with and without mucus; however they were not able to distinguish the coughs with wheeze. They concluded that healthcare professionals can recognize some of the qualities of cough sounds but they are not very successful in making a diagnosis [20].

Hirtum et al. examined the fundamental frequency of healthy and unhealthy coughs from human and pigs. They found that the fundamental frequency of the cough of healthy groups was higher than infected groups [21]. Ferrari et al. also found similar results to Hirtum. They compared the cough of healthy pigs with pigs with infected lungs. Results showed that healthy pigs have a significantly higher peak frequency and shorter duration of cough compared to the infected pigs [22].

Bianchi et al. studied the distribution of cough peak flows (CPFs) in healthy children between 4-18 years old. They found that CPF values are higher in older children than younger ones. The purpose of this study was to provide the health care society the CPF level for paediatric populations to prevent the risk of acute respiratory diseases for younger children [23].

Another study investigated the effects of paralyzed vocal fold on the intensity of coughing. Murty et al. compared the airflow of voluntary cough in patients with vocal fold palsy to a healthy group. They concluded that there was no significant difference between the two groups in peak airflow. The only difference was the time it took each 
group to reach the peak airflow. According to their findings, it took patients with vocal fold palsy longer to reach the peak airflow than the healthy group. These findings might be useful in detecting the presence of vocal fold palsy by testing the airflow in voluntary cough; however, it cannot identify the position of the lesion [24].

As mentioned in Chapter 1, cough sound discrimination is part of the second step involved in a typical cough sound analysis system (Figure 1.1). The first step of the cough sound analysis system is cough sound detection. Cough sound detection involves detecting cough from other environmental and biological sounds and will be discussed in the following section of this chapter.

\subsection{Cough Detection}

Cough is the result of rapid changes in airflow generated by the contractions of different muscles, such as the chest wall, abdomen, diaphragm, and larynx. Hence, there are two types of signal that can be monitored: sound and movement. The list of different sensors for each signal is presented in Table 2 [25]. In this section, cough sound monitoring and detection will be investigated in greater detail. 
Table 2: Cough monitoring sensors [25]

\begin{tabular}{|l|l|}
\hline Sound & Free-field microphone \\
& Air-coupled microphone \\
& Contact microphone \\
\hline Movement & Electromyography \\
& Accelerometer \\
& Induction plethysmography \\
\hline
\end{tabular}

Cough sound detection involves collecting various sound types using microphones and extracting unique features specific to each type of sound collected (such as speech, laughter, snore, cough) and performing cough detection based on the extracted features $[26][27]$. There are three main steps involved in a typical sound detection system as illustrated in Figure 2.1. These three steps, which are sound acquisition, feature extraction algorithm and classification, will be discussed in the following two sections of this chapter.

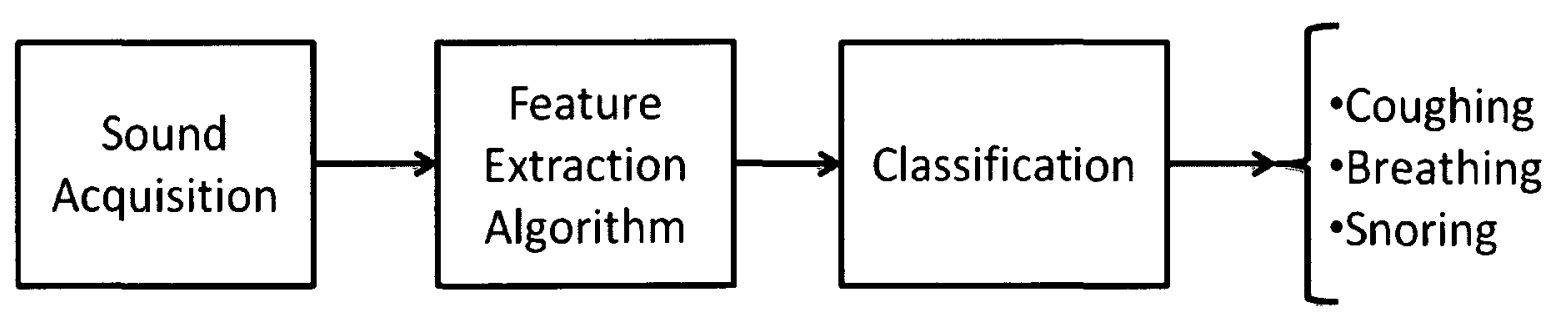

Figure 2.1: Sound Detection Algorithm 


\subsubsection{Sound Acquisition}

Sound acquisition is the first step in a typical sound detection system. It involves acquiring patient sounds using a sound acquisition device such as a microphone. The sound acquisition device can either be installed on a stationary platform or on a mobile device. Figure 2.2 shows different methods of sound acquisition from a patient. The sound acquisition algorithm could be different for indoor monitoring and outdoor monitoring; however, the concept remains the same.

For the stationary platforms, the sound acquisition device can be attached to the ceiling or wall of a patient's home, installed on bed headboards, or embedded in a bed mat. For mobile devices, the sound acquisition device can be embedded in portable devices or smart phones, such as a Blackberry ${ }^{\circledR}$ device. These portable devices could then be carried by the patients in the house and outdoors.

Cough monitoring and cough detection have been a topic of study since the 1960s [1]. Woolf and Rosenberg proposed a method of cough assessment under clinical conditions by a tape recorder system [28]. They proposed a technique to assess cough suppressants by counting continuously recorded cough sounds. 


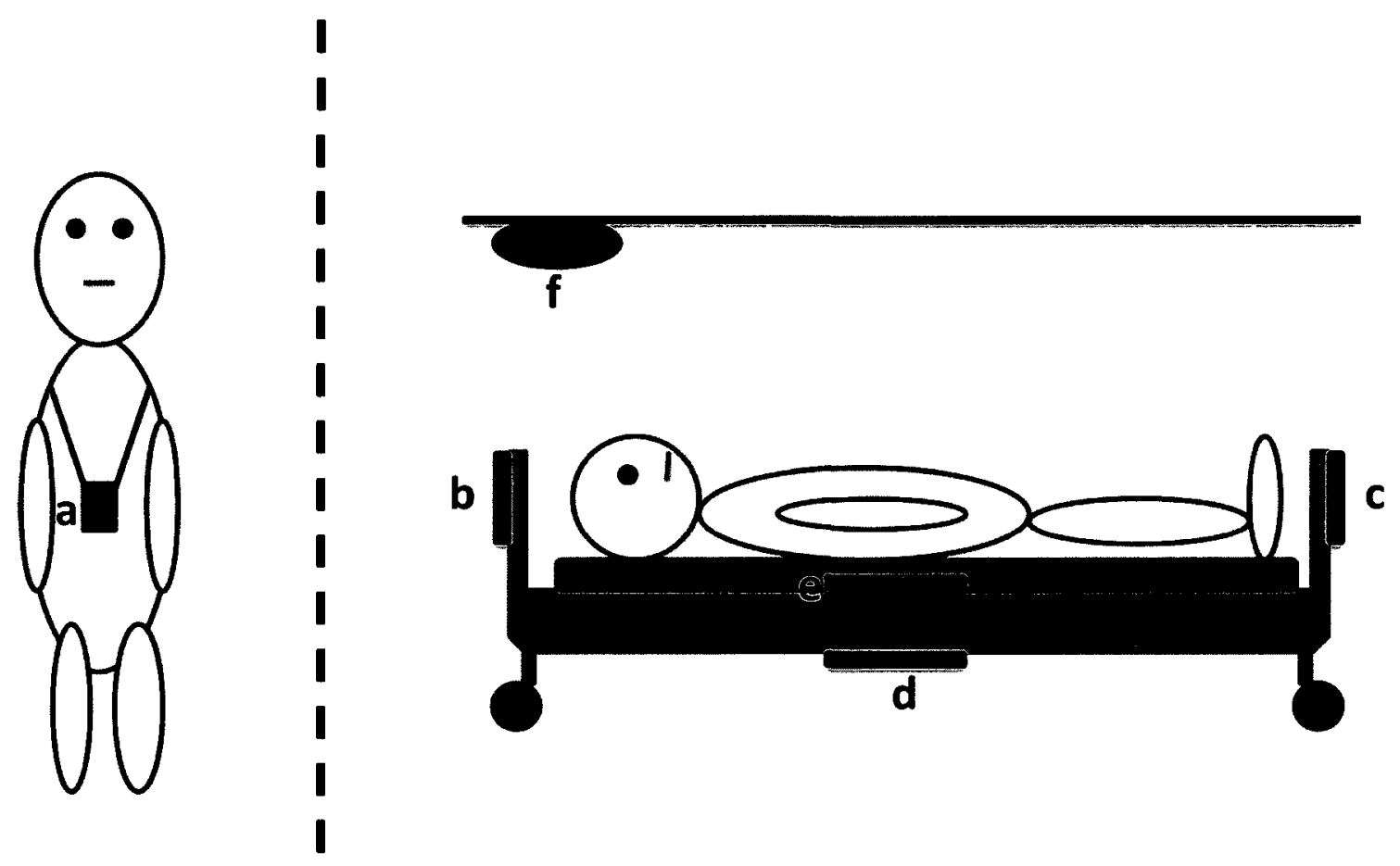

Figure 2.2: Sound acquisition device installed on a) a portable device, b,c) bed headboards, d) underneath the bed, e) embedded in bed mat, and f) the ceiling.

Ser et al. used microphone arrays to design a wearable health monitoring system to detect wheezy sounds. They used two kinds of microphones: two regular air-conductive microphones and one bone conductive microphone. They captured the breathing sounds by air-conductive microphones and lung sounds by bone conductive microphone. Their system showed good responses in different Signal-to-Noise Ratio (SNR) conditions. Their system was implemented on a Personal Digital Assistance (PDA) device and was tested by real data [29]. 
Matos et al. used a portable sound acquisition device to monitor cough sounds over 24 hours [1]. They proposed a novel system to monitor cough frequency by acquiring the cough signal via a miniature microphone placed around the patient's neck. Their system had a user-friendly graphical user interface that allowed its use by healthcare professionals in clinical practice. They created a cough database from 10 patients with different respiratory diseases to train their system. It was stated that by using more data to train the system and taking the common characteristics of the cough into account, they can model a more precise system for a specific type of cough [1].

Hata et al. proposed a healthcare monitoring system that consists of three systems: (1) heart rate detection, (2) respiration detection, and (3) cough with phlegm to suction detection. They used three non-contacted sensors for each system. For the heart rate detection, they used an ultrasonic oscillosensor attached to the bed. They used air pressure with an air tube in the bed mat to detect respiration. For collecting cough sounds, they used a PCM recorder with microphone. They used Power Spectral Density (PSD) ratios of different frequency bands to detect cough sounds from other types of sounds. [30][31].

\subsubsection{Feature Extraction Algorithm and Classification Techniques}

The next step in the sound detection system as depicted in Figure 2.1, involves extracting the unique features of specific sounds and classifying them into different types of sounds such as speech, cough, breathing, snoring and much more. 
One of the most important types of parametric representation used in speech recognition is Mel Frequency Cepstral Coefficient (MFCC). MFCC describes the characteristics of the audio data frame in the cepstral domain. It shows the short-term power spectrum of a sound. Matos et al. proposed an algorithm to detect cough sounds from non-cough events by extracting MFCC as a feature [32]. They used a Hidden Markov Model (HMM) as their classifier. Their automated cough detection was able to detect a high percentage $(82 \%)$ of cough events. However, the detection rate was dependent on the energy of the signal. The detection rate decreased for lower energy signals [32].

In another study, Shin et al. used features such as Energy Cepstral Coefficients (ECC) and MFCC to develop an automated system that can monitor and detect cough sounds [33]. They compared cough sounds to other types of sounds such as Japanese vowels, tambourine sound, thunder and dog barking. It was found that cough sound had a higher energy level at frequency ranges between $400 \mathrm{~Hz}$ and $1.6 \mathrm{kHz}$ compared to other types of sounds. By using a band-pass filter with cut-off frequencies at $400 \mathrm{~Hz}$ and $1.6 \mathrm{kHz}$, they were able to discriminate between some of the sounds such as the tambourine sound. In addition, their hybrid model, which consisted of an artificial neural network (ANN) and an HMM mode, was able to distinguish cough sounds from other environmental sounds. Their model had higher performance at Signal-to-Noise Ratio (SNR) levels below $5 \mathrm{~dB}$ as compared to a conventional HMM which uses only MFCC as a feature [33]. 
Abaza et al. used various features in both time and frequency domain to classify cough sounds recorded from both male and female participants with normal and abnormal lung function. Some of the time domain features were peak cough flow, average cough flow, and total cough volume and some of the frequency domain features were total energy, dominant frequency, and peak power [34].

In another study, Hirtum and Berckmans classified spontaneous coughs versus voluntary coughs. They created a cough sound database from three unhealthy and nine healthy subjects between 20 and 30 years old. They looked at the PSD of each cough sound and used different transformations of the cough PSD-vector for the classification [35].

Martinek et al. proposed an ambulatory cough monitoring system to detect cough sounds from non-cough sounds. They performed both spectral and non-linear analysis (i.e. sample entropy) on both types of sounds where sample entropy measures the unpredictability and irregularity of signals. From their analysis, they discovered that cough showed more irregularity when compared to speech [36].

The sound detection system such as the cough sound analysis system depicted in Figure 1.1 could be integrated as part of a smart home or a Remote Patient Monitoring system (RPM) in order to assist healthcare professionals and improve the quality of the healthcare system as a whole. The following section describes smart home technology and its significance in the healthcare society. 


\subsection{Smart Home Technology}

Smart home technologies provide healthcare professionals with technologies that can assist in the care of the elderly. Monitoring cough could be part of a smart home or could be embedded in smart phones. In the smart home, cough monitoring can be done through the use of microphone arrays. In smart phones, algorithms can be embedded into the phone software to collect ambient sound and analyze cough sounds. This section reviews various sensor technologies that are used in smart homes to monitor their occupants.

Arcelus et al. described a prototype home equipped with a variety of sensor technologies such as: magnetic switches, thermistors, accelerometers, microphone arrays, and pressure sensitive mats [5]. Sensors were used to either monitor patient behaviour or activity, or used to monitor specific health signals from the patient and hence, aid patients to live independently. For instance, microphone arrays were used in smart homes in order to detect and monitor different sounds such as coughing, snoring, and breathing and transmit abnormal sounds to a monitoring station. An artificial intelligence (AI) system was used in order to make decisions based on all the information gathered from all of the sensors throughout the smart home [5].

There is also a lot of research performed on locating the resident of the smart home or smart office. In [37], Lu et al. established ambient intelligent applications in a smart home. They presented a location-recognition algorithm using a Bayesian-Network-based fusion engine. Their design was based on various wireless sensor technologies in order to 
collect informative features. The features were ranked based on their usefulness in estimating activities of interest. They included the context-awareness into the activity recognition using location information. The goal of this work was to utilize the contextaware attentive services to fulfill real human needs.

In [38], Kim et al. presented a location-recognition algorithm using infrared sensors for indoor location awareness system. The sensors were installed on the ceiling in order to determine the location of the resident. The location accuracy of the system was dependent on the number and location of the sensors. The lower accuracy of the system was outweighed by the fact that there was no need for the residents to carry any devices with them, which made the location-recognition system very useful and practical.

A lot of studies have been working towards improving the sensor technologies used in Remote Patient Monitoring systems (RPM) and smart homes, such as using microphone arrays to monitor and detect sounds.

In [39], Vacher et al. proposed a sound recognition system in smart homes. They used 8 microphones to detect, analyze and classify different sounds and speech utterances such as normal and distress French sentences. They performed two experiments. The first experiment was to detect the distress situation. They played a scenario using 10 speakers and calculated the global error rate to be $15.6 \%$. This result was expected to improve by taking into account the sounds generated in speech. The second experiment focused on 
noise cancellation. They performed noise cancellation techniques during the detection of distress sentences uttered by four speakers. The missed alarm rate was $27 \%$.

Zhang et al. presented a new approach to measure the heart rate using sound signals. They used an air conductive microphone to collect sound signals to estimate heart beat parameters. They collected data from certain activities, such as sitting, jumping, reading, and coughing. The participants were the researchers and students working in the laboratory. The results showed that this new method could be an alternative method to measuring the heart beat [40].

Andoh et al. monitored the sleep stages of their participants using heartbeats and their body motion using pneumatic microphone sensors named air cushion sensor. According to Andoh et al., a health monitoring system needs to fulfill the requirements outlined in Table 3 and they proposed a system that can meet those requirements [41].

Some of the information extracted from the mentioned sensors in smart homes or smart devices could in the future help with sound analysis. For example, cough information coupled with information from the pressure-sensitive mats could indicate whether the patient was in bed and whether they were lying or sitting when the cough event occurred. Also, cough information combined with body temperature sensor data can be helpful in the clinical diagnosis. 
Table 3: Perquisite requirements of health monitoring at home [41]

\begin{tabular}{|l|l|}
\hline Condifion & The normal operation of the device is done \\
\hline Ease of operation & by pushing three different buttons \\
\hline Non-invasive & $\begin{array}{l}\text { Sensing air cushion is located under the } \\
\text { bed cushion }\end{array}$ \\
\hline Stress free, both physically and mentally & Existence of the sensor is not noticeable, \\
& since it is located under the bed cushion \\
\hline Reasonable price & The device is reasonably priced \\
\hline
\end{tabular}

Smart home technologies and remote patient monitoring have gained a lot of attention recently since they help improve the healthcare system and also reduce the overall cost for both patients and healthcare facilities [42]. With all the new technologies involved in smart home patient monitoring, the quality of life of the elderly will improve and the cost of healthcare services will reduce in the long run. Furthermore, it will help the elderly people to live and stay independent. Elderly patients can decide where and how they want to live and at the same time take advantage of high quality healthcare services.

In Canada and all other countries, the aging population is growing as compared to other age groups [6][43]. This trend would make it challenging for hospitals and healthcare facilities to provide various medical services to the aging population [44][45]. Another factor that makes this issue more challenging is patients who suffer from chronic 
diseases, such as heart disease, stroke, cancer, chronic respiratory diseases and diabetes. Chronic diseases are long lasting and slow progressing diseases which need constant care and supervision [46][47][48]. Asthma and chronic obstructive pulmonary disease (COPD) are two common types of chronic respiratory diseases [49][50]. According to the World Health Organization (WHO) statistics in 2008, 300 million people suffer from asthma and COPD. The cost of constant care and hospitalization is very high for chronic diseases [51][52]. It is estimated that the cost of most common chronic diseases is more than $\$ 1$ trillion annually in America and it is predicted that the cost will increase to $\$ 6$ trillion by the middle of the century [53]. Due to the costly treatments, the rate of death related to chronic diseases is very high [54] in low and middle income countries [55][56].

As a consequence of the increase in the aging population, the need for smart homes and remote patient monitoring systems has increased to overcome the challenges associated with the aging population [57].

\subsection{Summary}

Coughing is one of the most common symptoms in most of the respiratory diseases. Therefore, cough monitoring, detection and discrimination have gained a lot of attention among researchers. Some of the cough related clinical information that can be provided to healthcare professionals are the number of cough sounds in one recording, cough frequency, and cough severity. The mentioned information, however, does not reveal the nature of the cough. As a consequence, the need for new techniques to discriminate 
cough sounds into different types (dry, wet, whooping, etc) has received significant attention recently. Providing the nature of cough to healthcare professionals could help them better prescribe a treatment process and drug selection for the patients.

Cough monitoring and detection are performed using various sensors installed on stationary and portable devices. These sensors are capable of recording ambient sounds and classifying the recorded sounds into different types, such as speech, cough and much more. These devices could be integrated into smart homes and Remote Patient Monitoring systems (RPM) which are equipped with other types of sensors such as magnetic switches, thermistors, accelerometers, microphone arrays, and pressure sensitive mats. Providing cough related information along with other information collected from the mentioned sensors could help improve the quality of diagnosis and treatment. For instance, providing cough related information along with the body temperature of the patient could be more valuable than providing only cough information. 


\section{Chapter 3:}

\section{Cough Sound Characteristics}

\subsection{Introduction}

This chapter describes the characteristics of cough sounds. The first part of this chapter summarizes the state of the art literature in the area. The second part of this chapter presents the findings of experiments that were conducted within the scope of the thesis. Chapter 3 begins with Section 3.2, which provides an introduction to the nature of cough, followed by an explanation for different types of cough in Section 3.3. Common characteristics of cough sounds will be explained in Section 3.4. Section 3.5 and Section 3.6 present the dry and wet cough signals characteristics respectively. The second phase of cough signal will be studied in more detail in Section 3.7. Finally Section 3.8 compares the characteristics of both dry and wet cough signals and highlights the differences between the two types of cough signal. 


\subsection{Cough Sounds}

Cough is one of the most common symptoms among all respiratory diseases. The main purpose of cough is to clear the breathing airways of foreign objects, secretions and mucus [58][59]. Depending on how often it occurs and its severity, cough may persist and become chronic in nature [60].

Coughing starts with the stimulation of cough receptors in the central airways by mechanical or chemical stimuli followed by the wide opening of the vocal cords to help pass more air into the lungs. At this point, the epiglottis closes off the windpipe and the abdominal and rib muscles contract simultaneously to increase the pressure behind the epiglottis. Subsequently, the air is forced to expel, which in turn creates a rushing sound as it quickly passes the vocal cords. The cough reflex happens until the foreign object is dislodged and comfortable breathing is once again possible [61]. The epiglottis, vocal cord and windpipe are depicted in Figure 3.1.

Cough characteristics and its acoustic features depend on the velocity of airflow, dimensions of the vocal tract and airways, and location of sound generated [61]. 


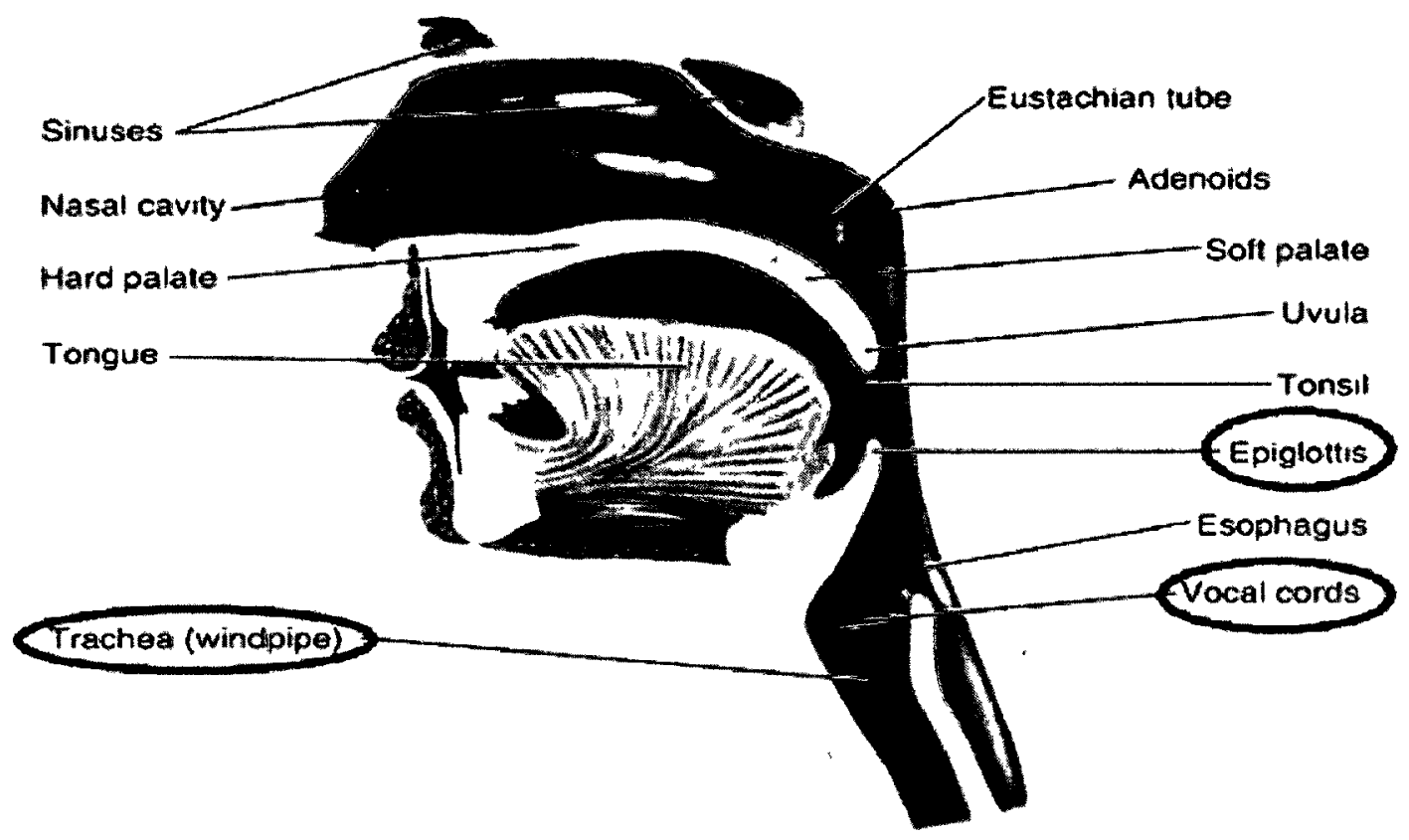

2007 RelayHealth andiof its affilates. All rights reserved.

Figure 3.1: Throat anatomy including vocal cord and epiglottis [62].

\subsection{Types of Cough}

Cough sounds can be classified by nature into two types: dry (non-productive) and wet (productive) [17][63]. Dry coughs, as the name indicates, are dry and therefore, might feel ticklish or cause irritation. On the other hand, wet coughs are wet and produce mucus that may be thick and have white or yellow color.

The nature of cough is important in pathological studies and for diagnostic purposes. Differentiation of dry and wet cough sounds however, is very subjective. Sometimes, it is difficult for patients to describe their cough sounds to healthcare professionals and this 
could make the diagnosis of disease and optimal prescription more difficult. As was mentioned in Chapter 2, work on automated cough monitoring systems has therefore increased in recent years especially in the context of smart home monitoring $[32][64][65][66]$.

\subsection{Common Characteristics of Cough Signal}

Morice et al. indicated that there are three main acoustic patterns of cough in the literature. The three patterns are shown in Figure 3.2, Figure 3.3, and Figure 3.4. The first pattern is a cough with three distinct phases, which is the most common pattern of cough.

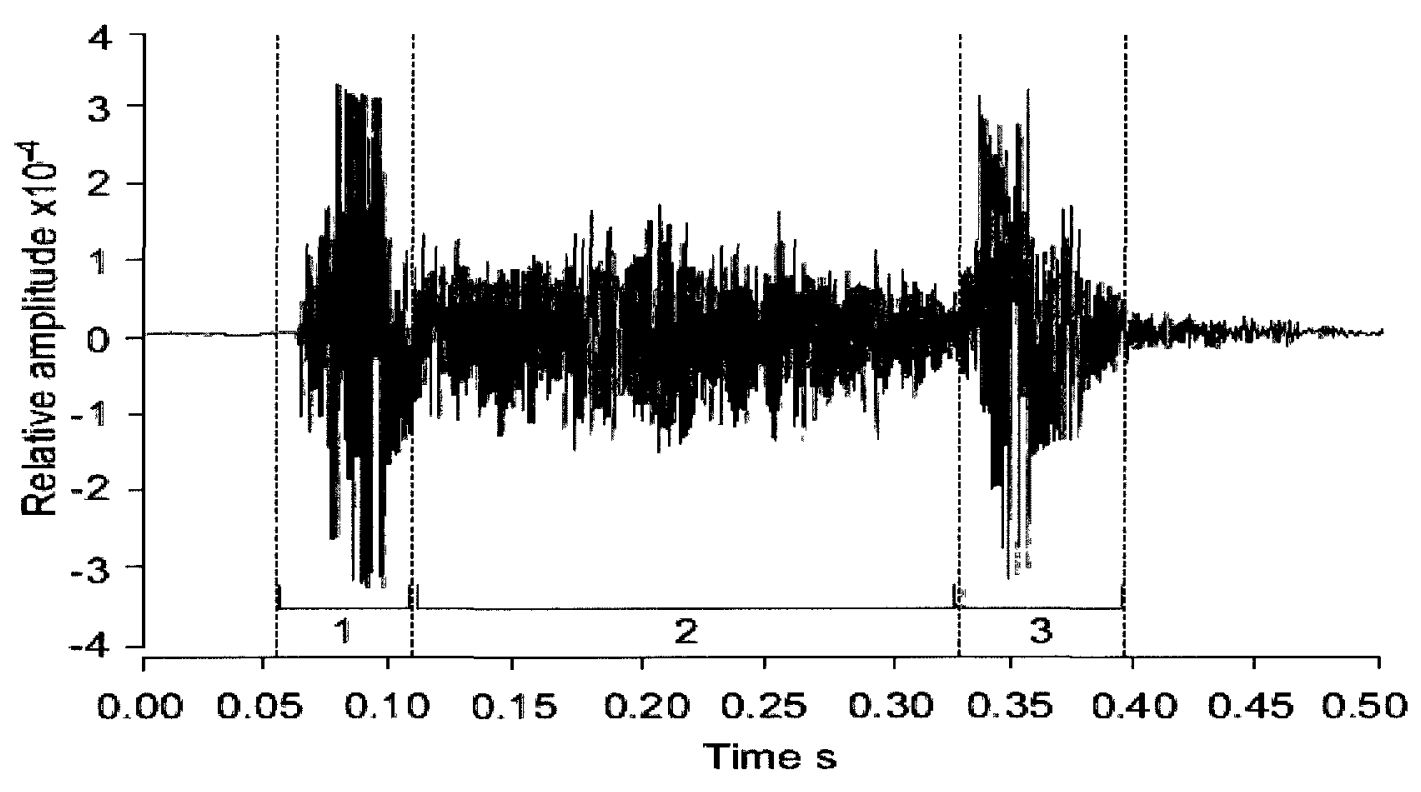

Figure 3.2: Three-phase cough (first pattern) (Taken with permission from [25]). 
The second pattern of cough signal consists of only two phases. Finally, the third type of cough signal is a peal cough [25]. In a peal cough pattern, the amplitude of the successive coughs decreases as lung volume decreases [67].

Korpas et al. explained the origin and process of each phase of cough. "First phase is the result of airway narrowing and bifurcations, which leads to turbulent airflow. Turbulent airflow produces vibrations of the airway and the surrounding lung tissue. In pathological conditions the airway narrowing is multiplied due to the local thickenings of the airway walls (inflammation, oedema, mucus collection), bronchoconstriction, fibrosis, etc."[16]. According to Korpas, the second phase shows the flow of airf in the trachea and collection of mucus in the trachea. The final phase has been described to be the vocal fold adduction at the end of the second phase of cough [16].

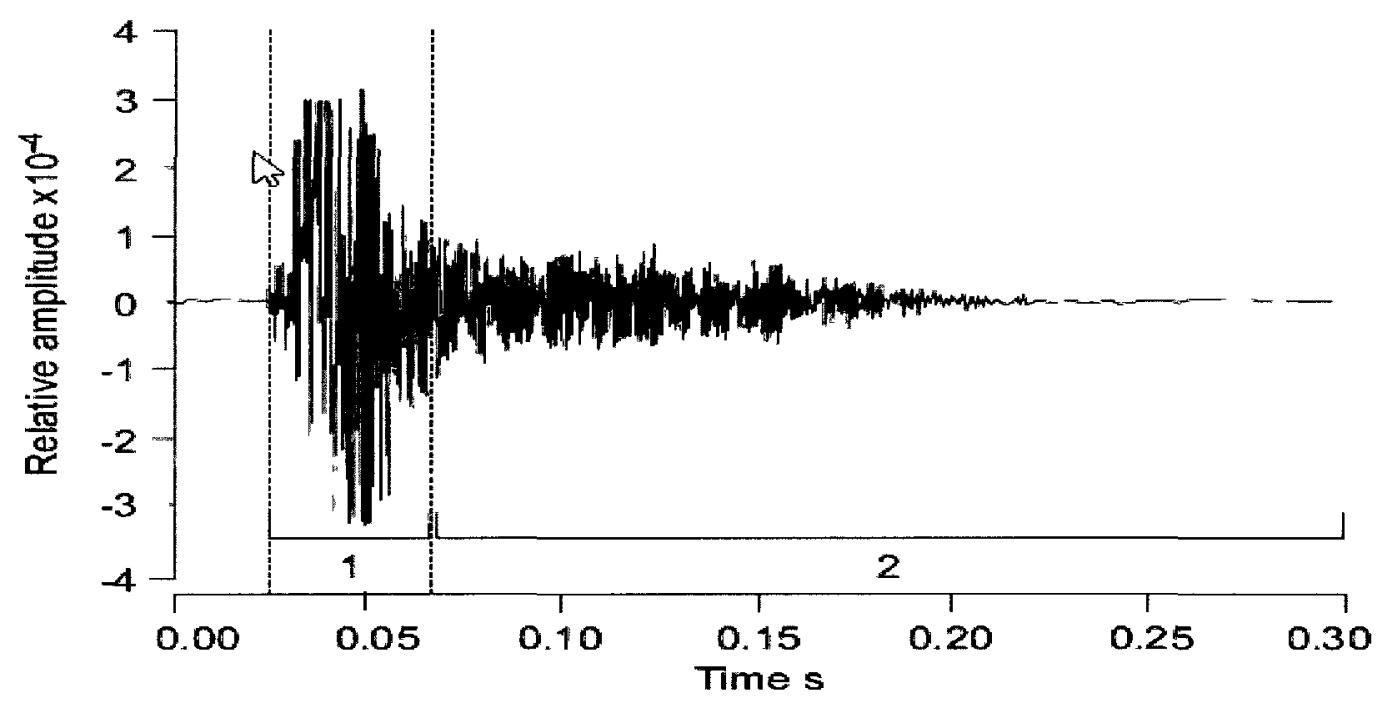

Figure 3.3: Two-phase cough (second pattern) Taken with permission from [25]). 


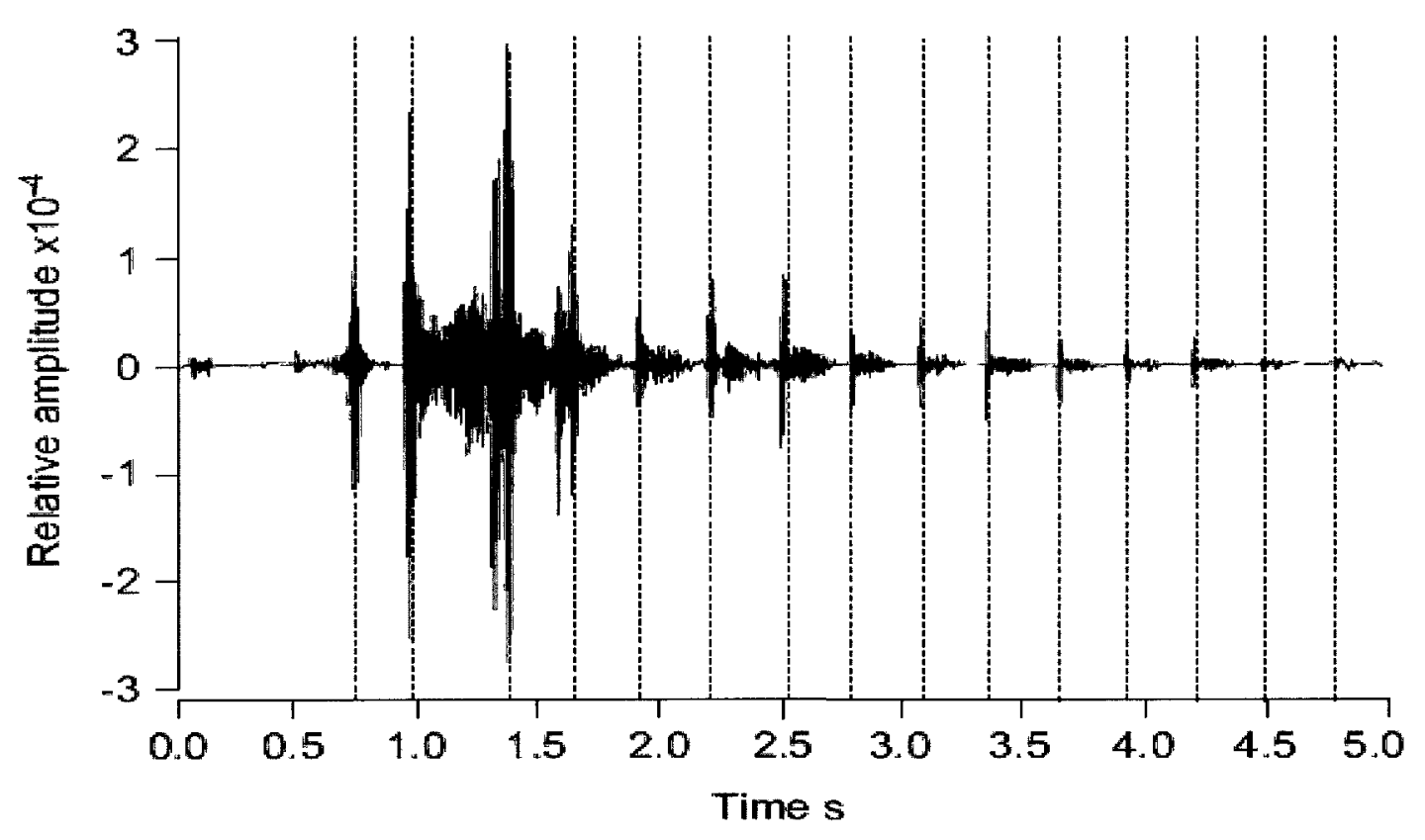

Figure 3.4: Peal cough (third pattern) (Taken with permission from [25]).

Doherty et al. analyzed the acoustic properties of cough in both time and frequency domain using voluntary and spontaneous cough on healthy subjects. They observed that the most common pattern of a cough is the three-phase cough. Eight out of 13 participants produced the three-phase cough, whereas three out of 13 participants produced the two-phase cough and only two subjects produced the peal cough [67].

Olia et al. studied acoustic features of voluntary coughs in healthy males and females. They studied the cough length, duration of each phase and the level of spectral energy in each phase of cough. The results are summarized in Table 4 [68]. 
Table 4: Acoustic properties of cough in healthy females and males [68]

\begin{tabular}{|l|l|l|}
\hline Couglo phase & Length & Spectrall energy \\
\hline Phase 1 & Longer in females than & Loudest energy was lower \\
& males & in males than females \\
\hline Phase 2 & No significant differences & $\begin{array}{l}\text { Energy was lower in males } \\
\text { than females at lower } \\
\text { frequencies }\end{array}$ \\
\hline Phase 3 & No significant differences & No significant differences \\
\hline
\end{tabular}

Figure 3.5a shows a cough signal, which consists of three distinct phases: Phase 1: initial opening burst, Phase 2: noisy airflow, and Phase 3: glottal closure [61][17][69].

The duration of a cough sound typically varies between $0.2-1$ second. The first phase of a cough signal has a higher amplitude and shorter duration than the other two phases. On the other hand, the second phase, Phase 2, tends to have a longer duration and lower amplitude than Phase 1 and Phase 3[70]. In some instances, Phase 3 is not visible in the cough signal and the amplitude of the signal decays slowly as the airflow reduces $[17][71]$. 
a)

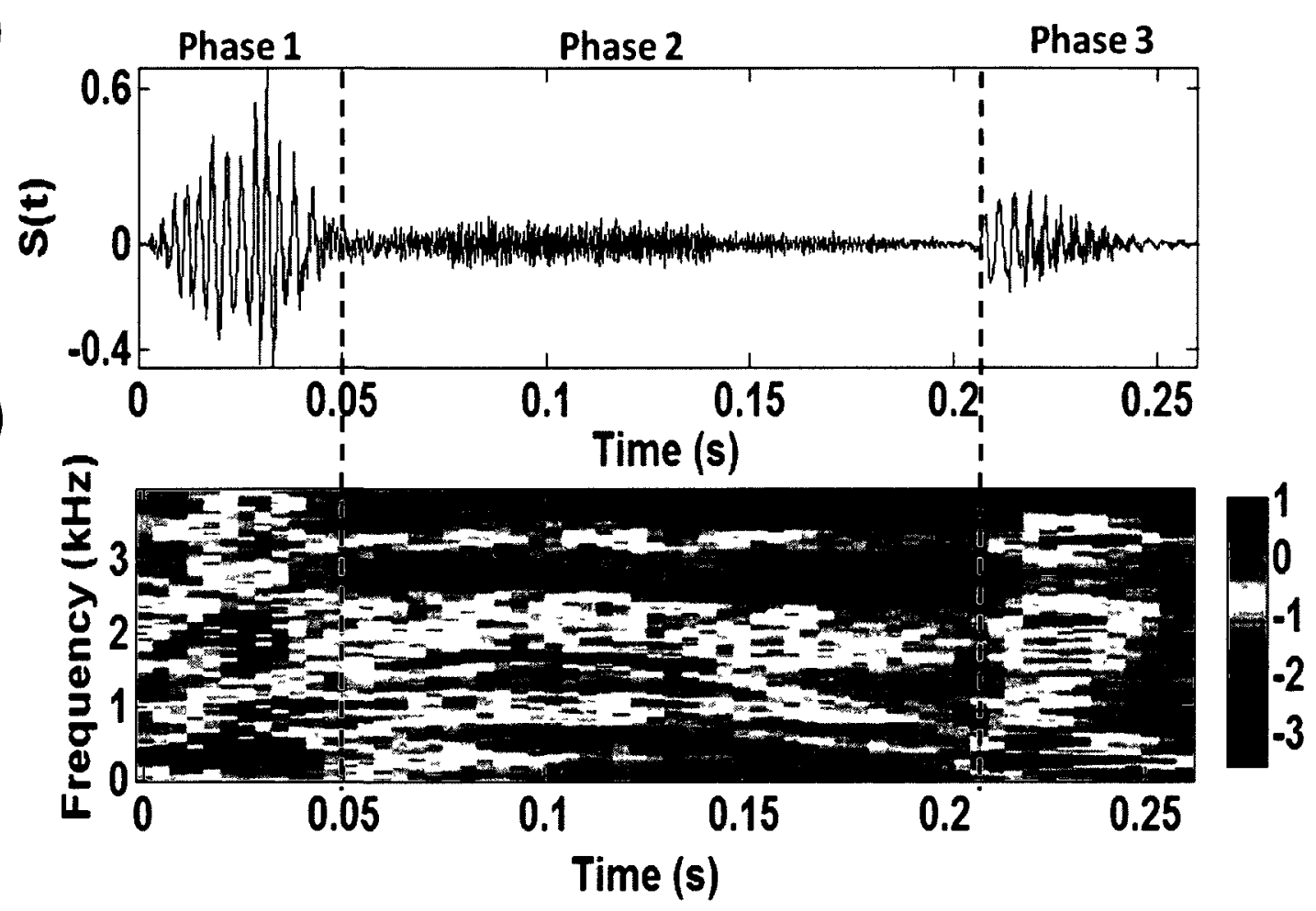

Figure 3.5: a) Dry cough signal with 3 phases: Phase 1, Phase 2, and Phase 3, b)

Spectrogram of dry cough signal.

Figure $3.5 \mathrm{~b}$ shows a sound spectrogram of a cough signal where the three phases of cough signal, Phase 1, Phase 2, and Phase 3, can be observed. The vertical axis shows the frequency distribution and the horizontal axis shows time in seconds. Each color represents the power of the signal at a specific time and frequency, with power decreasing from red to blue.

Phase 1 and Phase 3 tend to have similar characteristics in terms of frequency distribution. The only difference is that more power is observed in Phase 1 than in Phase 
3. On the other hand, power observed in Phase 2, in various frequency bands is less than Phase 1 and Phase 3, especially in lower frequencies.

\subsection{Characteristics of Dry Cough Signals}

A dry cough sound is dry and without any mucus or sputum [17]. Normally all the three phases are visible in a dry cough sound, as depicted in Figure 3.5 and Figure 3.6. As could be seen from Figure 3.5a, Phase 2 starts from the point of significant reduction in amplitude to where the amplitude starts to increase. Phase 1 and Phase 3 are determined to exist on the two ends of Phase 2. The characteristics of dry cough signals could be observed from both the time domain and frequency domain (spectrogram).

The spectrogram of a dry cough is shown in Figure 3.5b. As could be seen from the figure, Phases 1 and 3 of the dry cough sound contain high power at both lower and higher frequencies, whereas less power is observed in Phase 2 and especially at lower frequencies.

Furthermore, in dry sound spectrograms, few horizontal bands are observed in Phase 2. These horizontal lines show wheezing in the cough sounds [20]. Research has shown that identifying wheezes in cough sounds for health care professionals is not easy. However, wheezes can be easily identified in the sound spectrogram [20].

Analyzing dry cough signals in both time and frequency domain reveals many similarities between Phase 1 and Phase 3. They both exist for a short period of time and 
contain a wide range of frequencies. However, the behaviour of Phase 2 is different from those two phases.

Analysing dry cough signals in the time domain reveals important differences between the three phases of dry cough signals. One observation that can be made from Figure 3.6 is the fact that Phase 2 has a longer duration than the other two cough phases and oscillates between somewhat constant values with not much variation in its amplitude. Additionally, Phase 1, and in some cases Phase 3, has higher amplitude than that of Phase 2.

\subsection{Characteristics of Wet Cough Signals}

A wet cough is produced as a result of foreign bodies such as bacteria and viruses entering the body. Unlike dry cough sounds, wet cough sounds produce mucus and sputum [17].

A wet cough signal is shown in Figure 3.7a. The three phases are marked in the figure as Phase 1, Phase 2 and Phase 3. Phase 1 has higher amplitude than Phase 2; however, the amplitude difference between Phase 1 and Phase 2 is not very significant. Furthermore, one of the common characteristics of Phase 2 is its longer duration as compared to the other two phases (Phase 1 and Phase 3). 
a)

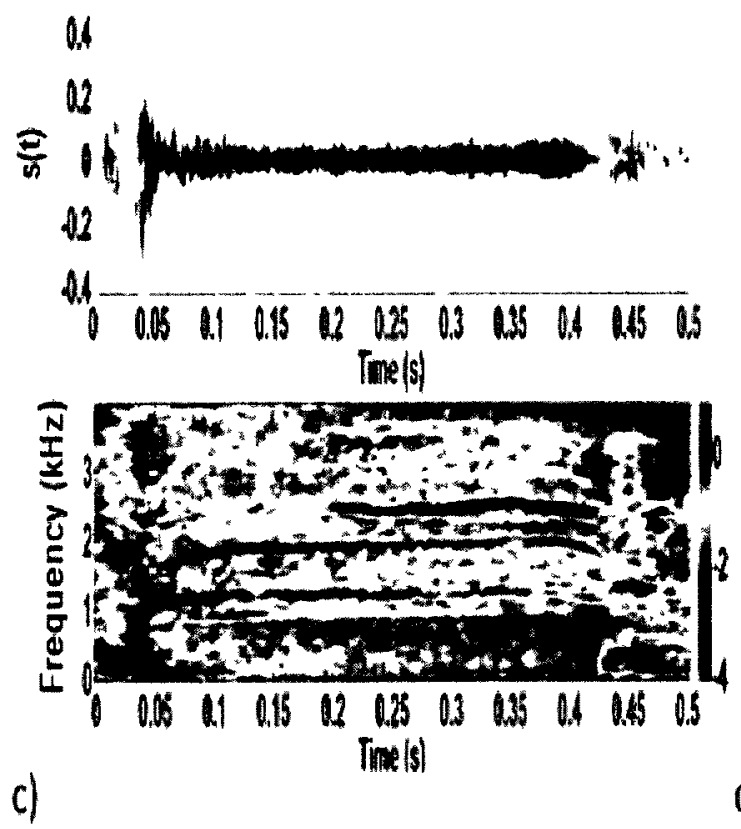

b)

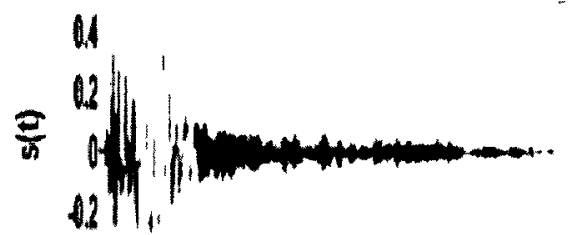

4.

1 ats 011 lif is

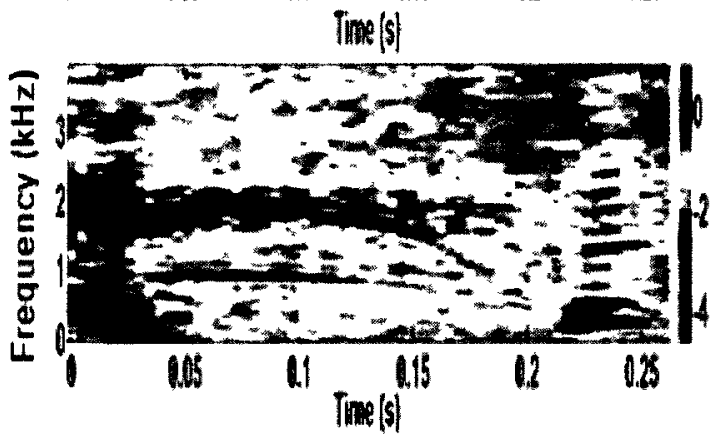

d)

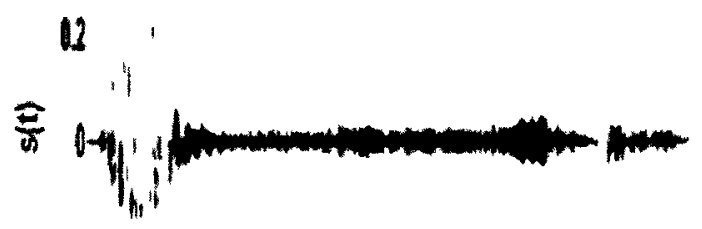

02

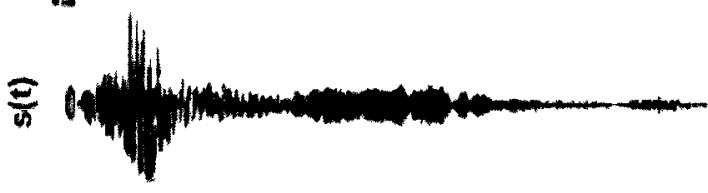

11

4

$\begin{array}{llllllll}1 & 0.6 & 0.1 & 1.15 & 0.2 & 0.5 & 13 & 0.35\end{array}$

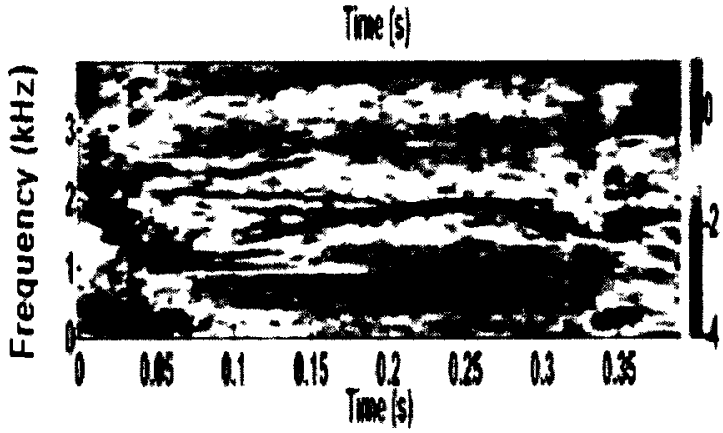

$\begin{array}{llllllll}1 & 0.6 & 0.1 & 0.15 & 02 & 0.6 & 0.3 & 0.5\end{array}$ Ineps]

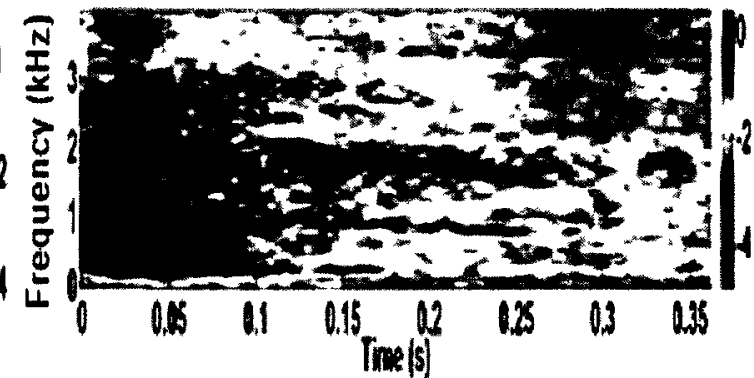

Figure 3.6: Dry cough signal along with its sound spectrogram a) Sample 1, b) Sample 2, c)

Sample 3, and d) Sample 4. 


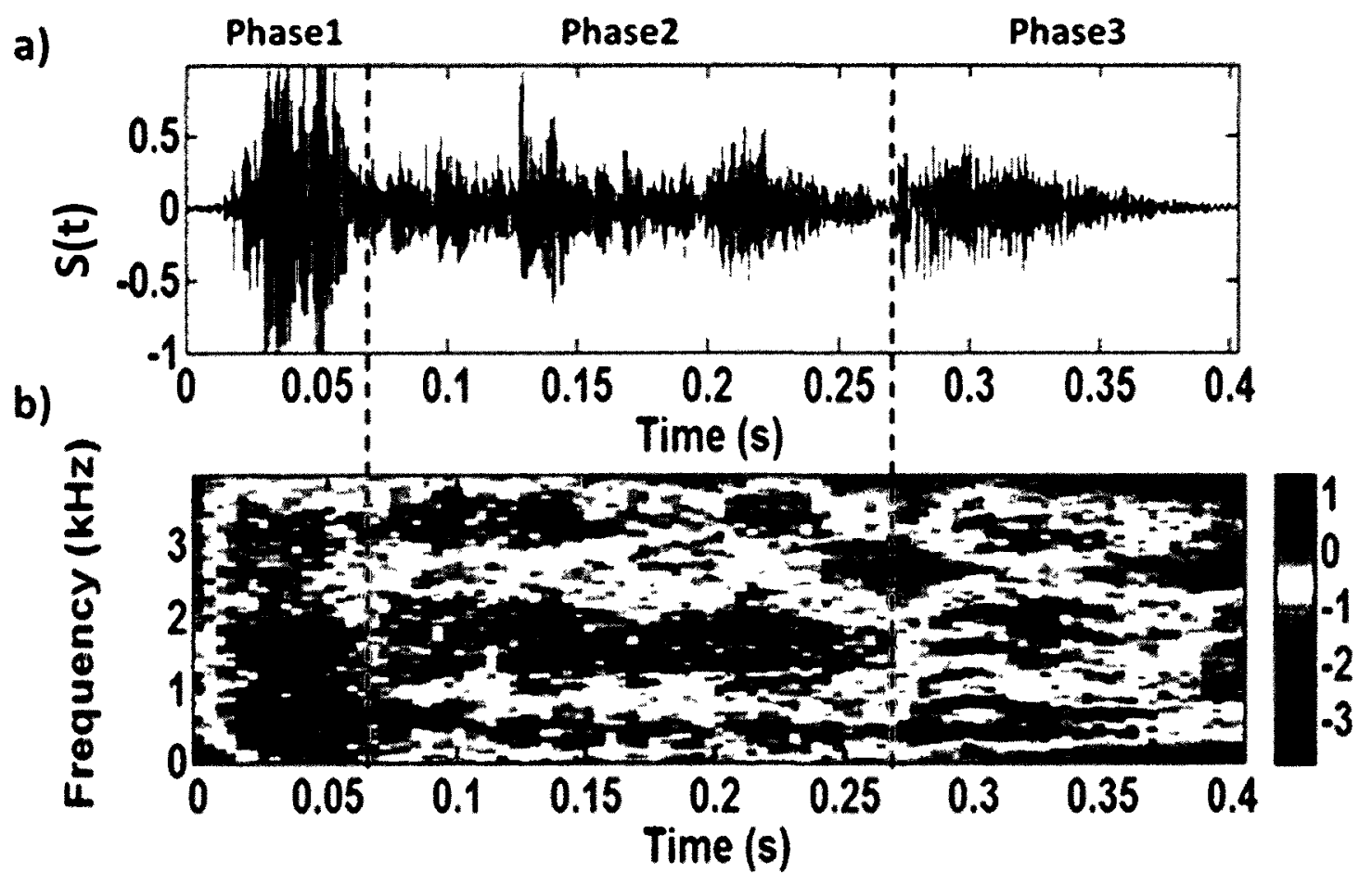

Figure 3.7: a) Wet cough signal with 3 phases: Phase 1, Phase 2, and Phase 3, b)

Spectrogram of wet cough signal.

Figure 3.8 shows four wet cough sounds along with their sound spectrograms. As can been seen from Figure 3.8, the three phases are not that visible in wet cough signals as compared to dry cough signals. Furthermore, in the sound spectrogram, detecting Phase 1, Phase 2, and Phase 3 is also not that clear. In addition, there is not much amplitude difference between the three phases. From the observations made above, it is clear that phase detection for wet coughs is more challenging than dry coughs. 
During Phase 2 of a wet cough, the signal oscillates randomly between different extremes and a large amount of amplitude variation can be observed. This is also reflected in its sound spectrograms.

One last observation that could be made from the Phase 2 spectrogram is the existence of a few vertical lines. Those vertical lines indicate interruptions in sound, caused by the mucus in wet cough sounds causing its spectrograms to be discontinuous $[17][19][20]$.

\subsection{Cough Signal Phase 2 Analysis}

From Section 3.5 and 3.6 observations, it is obvious that most of the differences between dry and wet cough sounds are in their Phase 2. Therefore, in this section, Phase 2 of both dry and wet cough signals will be investigated in more details.

In Section 3.5, it was mentioned that there is not much amplitude variation in Phase 2 of dry cough signals. In Section 3.6, however, it was observed that a significant amount of amplitude variation exists in Phase 2 of wet cough signals. Comparing the sound spectrogram of both dry and wet cough sounds revealed that Phase 2 of wet cough sounds has more power at lower frequencies than Phase 2 of dry cough sounds. Based on these observations, it was decided to explore the Phase 2 characteristics at lower frequencies in more detail. 
a)

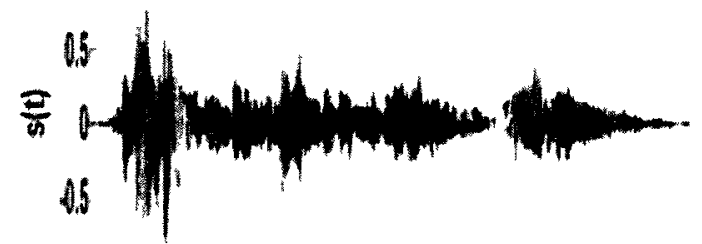

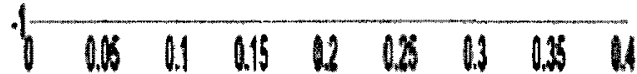
$\operatorname{Tin}(\mathrm{s})$

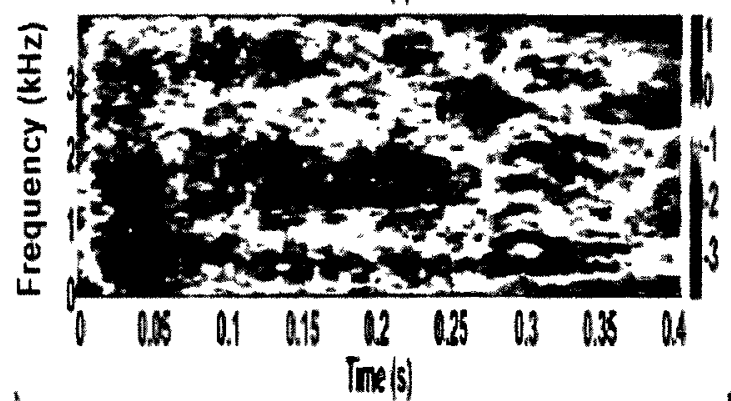

c)

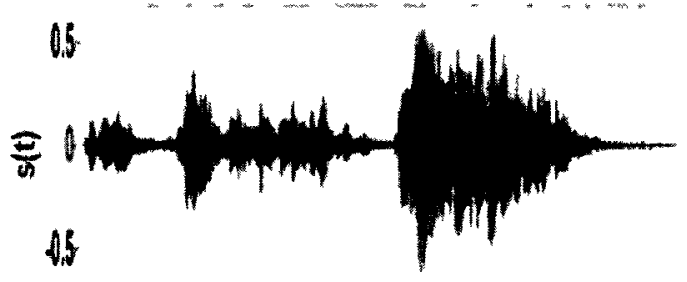

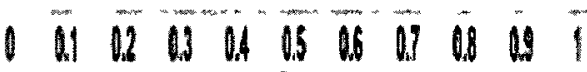
Trimels

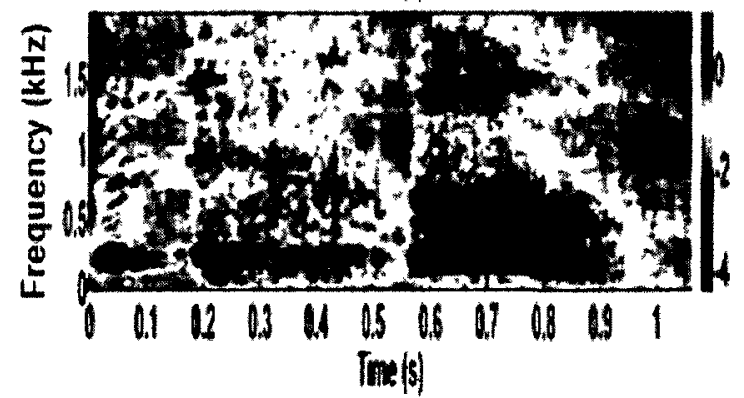

b)

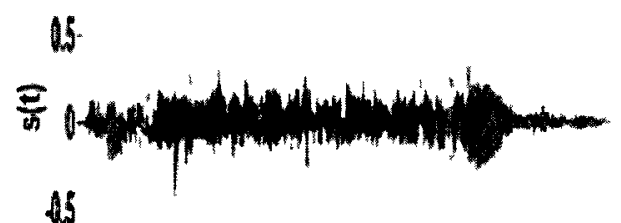

As

1011 is a is ime(s)

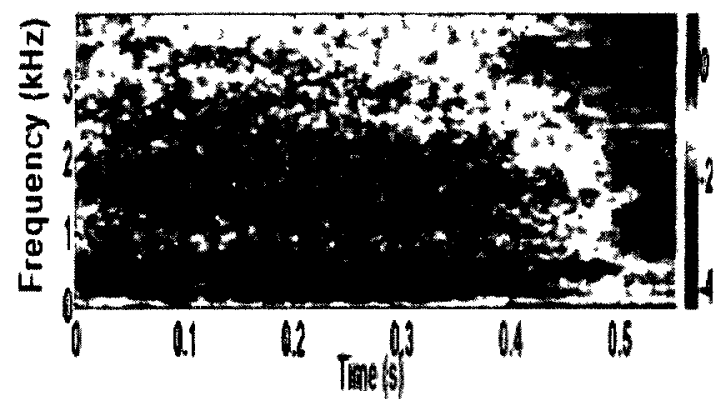

d)

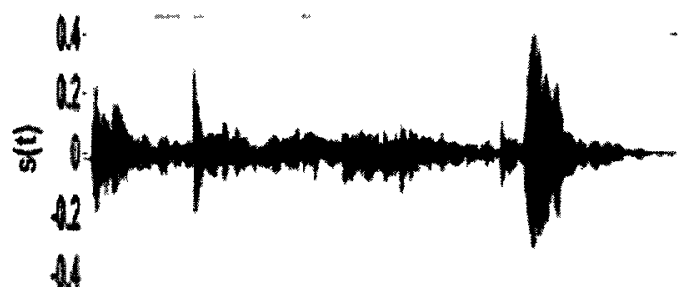

$\begin{array}{lllllllll}1 & 01 & 0.2 & 0.3 & 41 & 0.5 & 0.6 & 0.7 & 18\end{array}$ Tnels

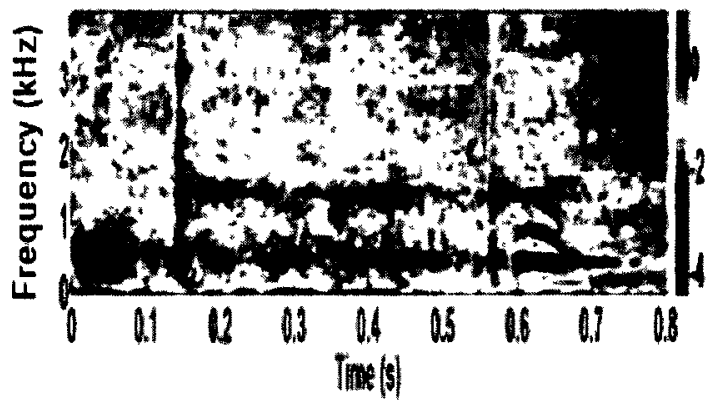

Figure 3.8: Wet cough signal along with its sound spectrogram a) Sample 1, b) Sample 2, c) Sample 3, and d) Sample 4. 
A low-pass filter with a cut-off frequency of $500 \mathrm{~Hz}$ was used to filter out all the frequency components above $500 \mathrm{~Hz}$ as depicted in Figure 3.9.

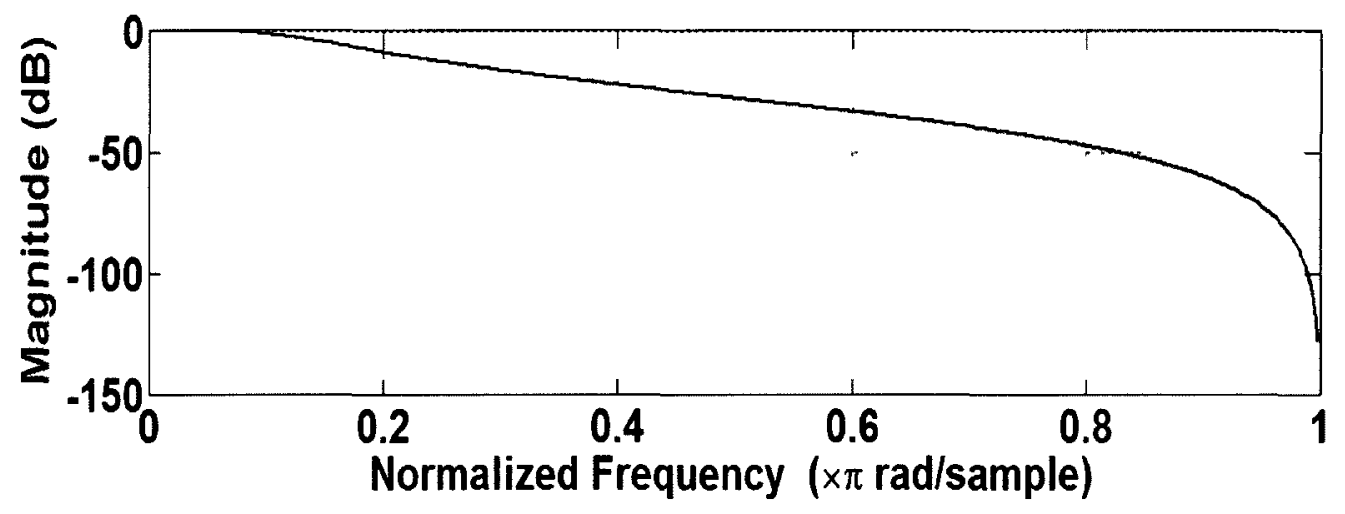

Figure 3.9: Magnitude response of a low-pass filter with cut-off frequency $500 \mathrm{~Hz}$.

Both dry and wet cough signals were passed through the low-pass filter, in order to study their behaviour at lower frequencies. Figure 3.10 shows two examples of dry cough signals (Figure 3.10a and Figure 3.10c) along with their low-pass filtered signals (Figure $3.10 \mathrm{~b}$ and Figure 3.10d). 
a)

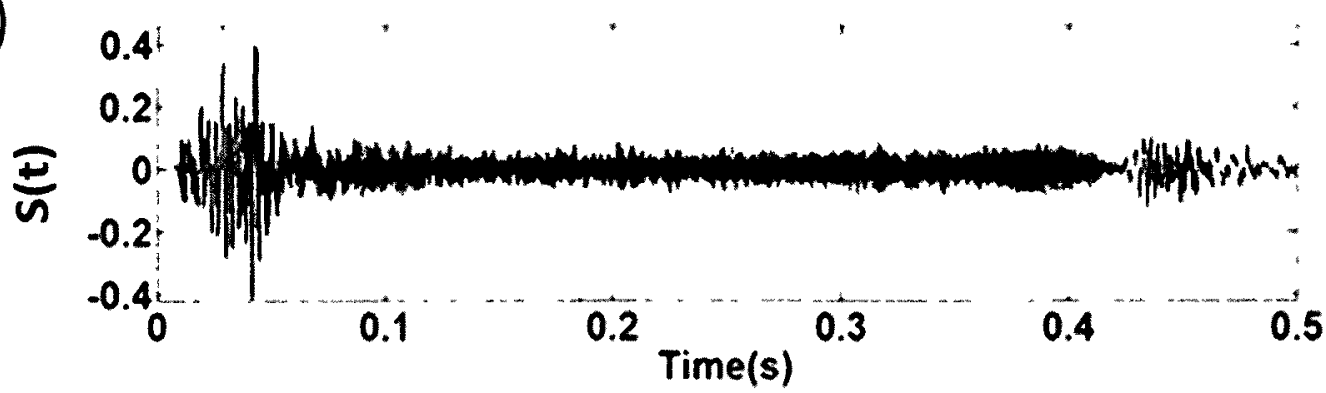

b)

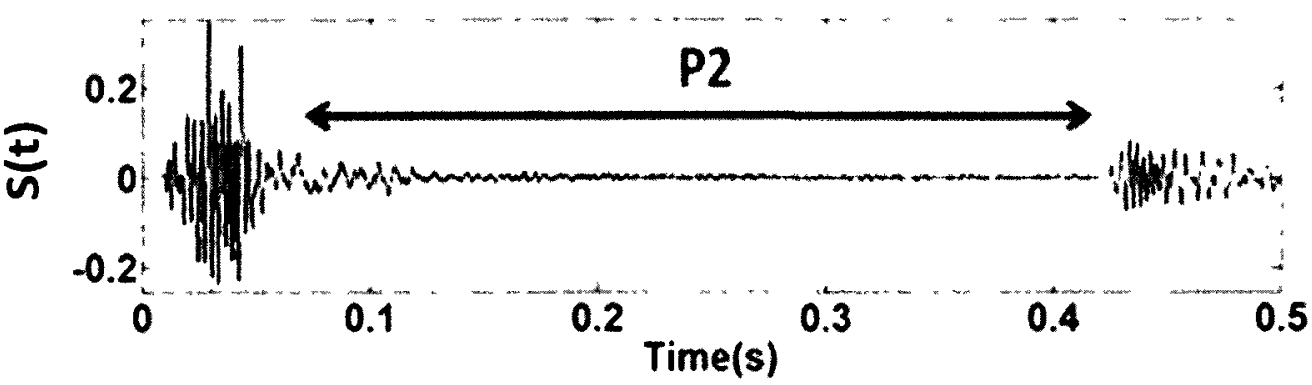

c)

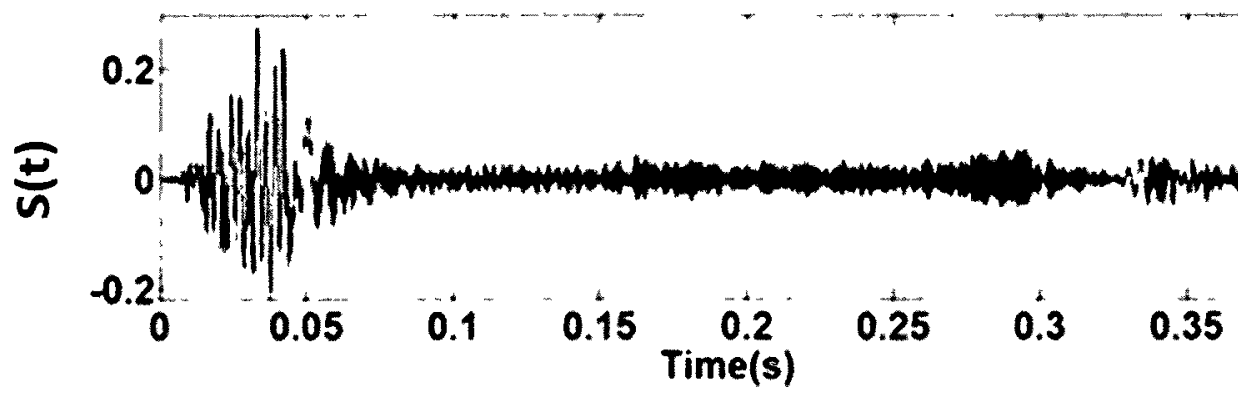

d)

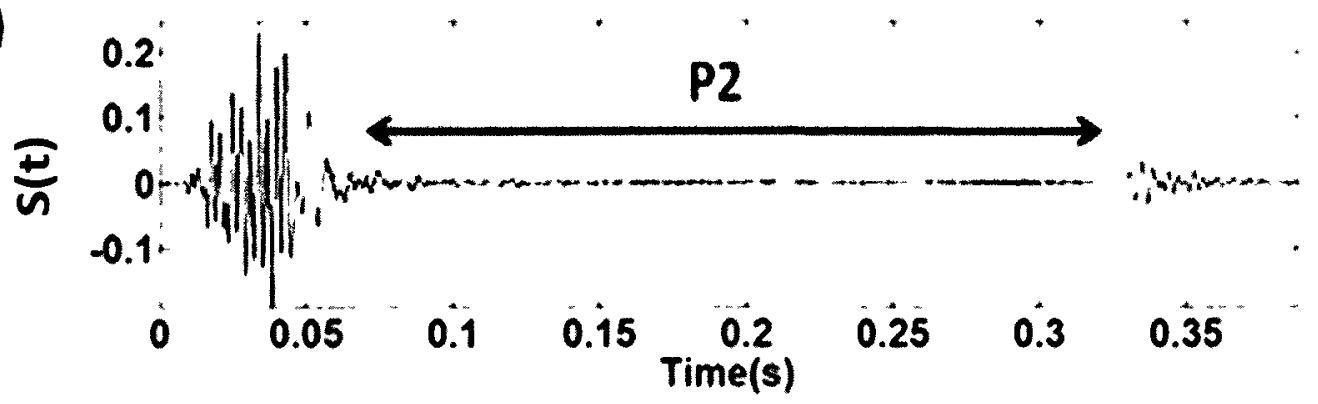

Figure 3.10: a) Dry cough sample 1, b) Low-pass filtered signal of dry cough sample 1, c) Dry cough sample 2, and d) Low-pass filtered signal of dry cough sample 2. 
Similar to Figure 3.10, Figure 3.11 shows two examples of wet cough signal (Figure 3.11a and Figure 3.11c) along with their low-pass filtered signals (Figure $3.11 \mathrm{~b}$ and Figure 3.11d).

As it was predicted, for dry cough sounds, most of the signal in Phase 2 was attenuated after passing through the low-pass filter. However Phase 1 and Phase 3 were still visible after passing through the low-pass filter.

On the other hand, unlike dry cough sounds, Phase 2 of wet cough sounds is visible, even though they were passed through a low-pass filter. Also the amplitude variation in Phase 2 can still be observed for wet cough signals. 
a)

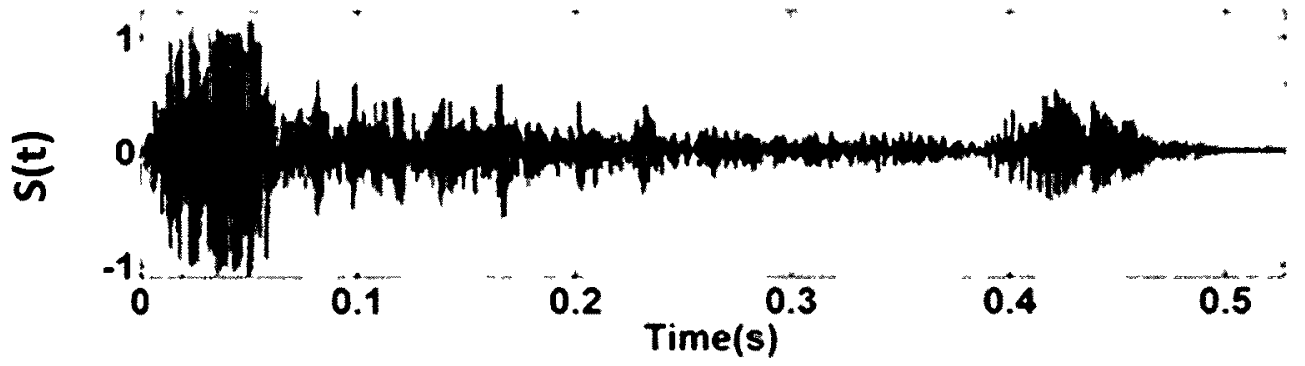

b)

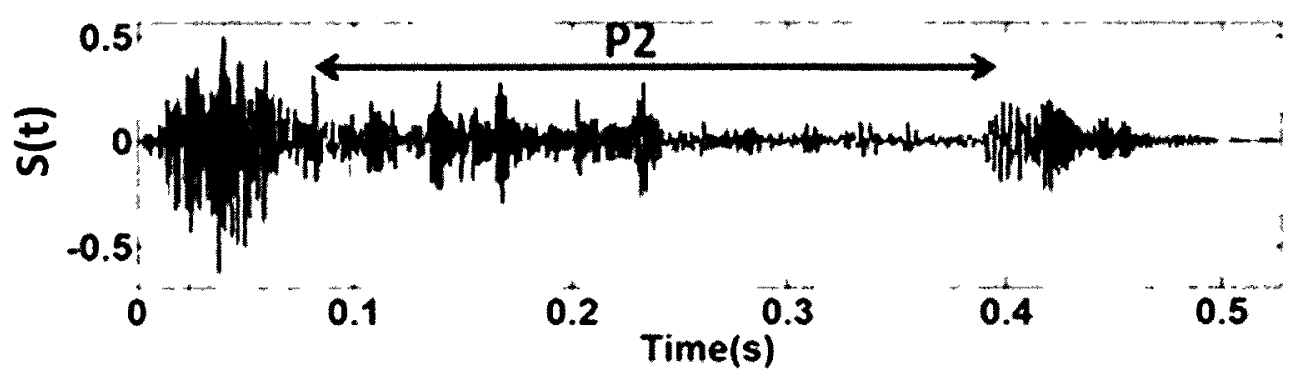

c)

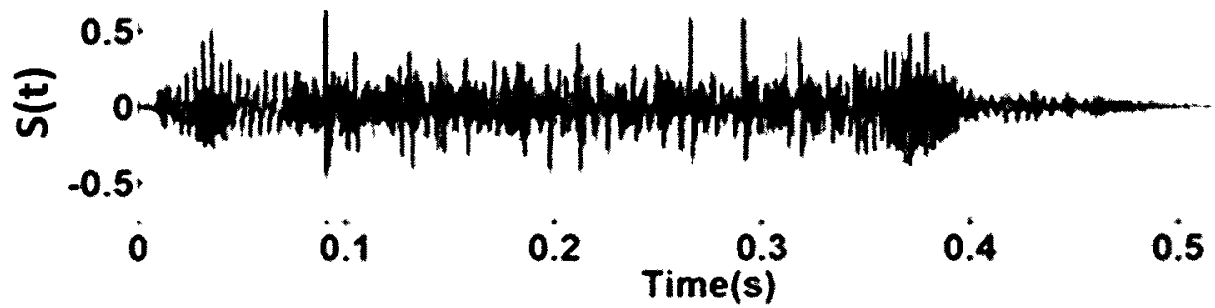

d)

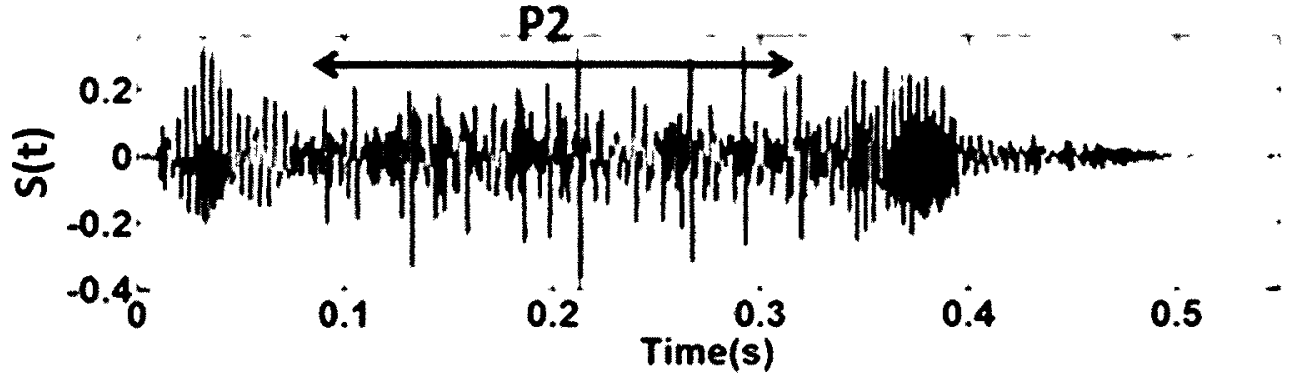

Figure 3.11: a) Wet cough sample 1, b) Low-pass filtered signal of wet cough sample 1, c) Wet cough sample 2, and d) Low-pass filtered signal of wet cough sample 2. 


\subsection{Conclusion}

In this chapter common characteristics of both dry and wet cough signals were explained. In Sections 3.5 and 3.6, dry and wet cough signals were analyzed in both time and frequency domains.

One of the observations was that the discrimination of phases for wet cough signals was more challenging than dry cough signals. The amplitude differences between Phase 1, Phase 2 and Phase 3 were not significant in most of the cases and thus, determining the beginning of each phase was more difficult in wet cough signals.

The amplitude variations between the three phases for wet cough signals were less than that of dry cough signals; nevertheless, the amplitude variations in Phase 2 of wet cough signal were greater than Phase 2 of dry cough signal.

The next observation was that more power was observed at lower frequencies of Phase 2 of wet cough signals than that of dry cough signals. This observation was explored in greater detail in Section 3.7 where both dry and wet cough signals were passed through a low-pass filter and the resulting Phase 2 of both types of cough signals were studied. For wet cough signals, a visible Phase 2 was observed, whereas this phase had vanished for dry cough signals. 


\section{Chapter 4:}

\section{Data Acquisition and Pre-Processing}

\section{Algorithm}

\subsection{Introduction}

In this chapter, data acquisition and the pre-processing algorithm used in this research are explained. Section 4.2 explains how the cough sound database used in this research was gathered and verified by medical professionals. Section 4.3 describes the segmentation process and how each cough recording was divided into cough episodes. Finally, section 4.4 explains the pre-processing algorithm that was applied on each sample of the cough sound database. 


\subsection{Cough Sound Database}

A cough sound database was created from cough samples obtained from many sources such as: Sound-Effect [72], Freesound project website [73], online samples provided by [20]. Also included are some cough samples that were recorded in the DSP lab at Carleton University from the author and other researchers from the team. The details of the database are listed in Table 5. There were 47 cough sounds, which included 21 female and 26 male cough sounds.

Table 5: Cough Sound Database

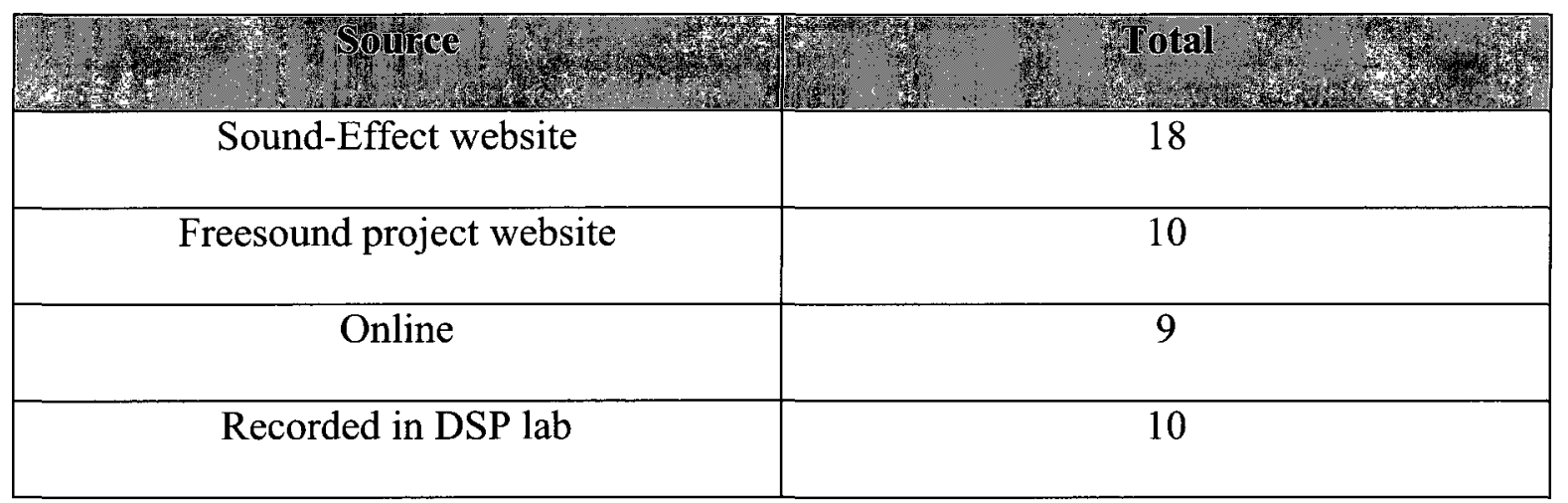

After listening to the cough sounds and using the information provided by the sources, the cough sounds were divided into two groups: dry and wet coughs. The type of each cough sound was further verified by medical professionals as well.

The scope of this research was to analyze the two types of cough sounds and discover the differences between the two. Therefore, nine highly dry and eight highly wet cough sounds were selected among all the cough sounds verified by medical professionals. 


\subsection{Segmentation}

Each cough recording consisted of multiple cough episodes [74]. Figure 4.1 shows a sample recording of a cough sound. As it can be seen from the figure, each cough recording might include 3 or more consecutive cough episodes.

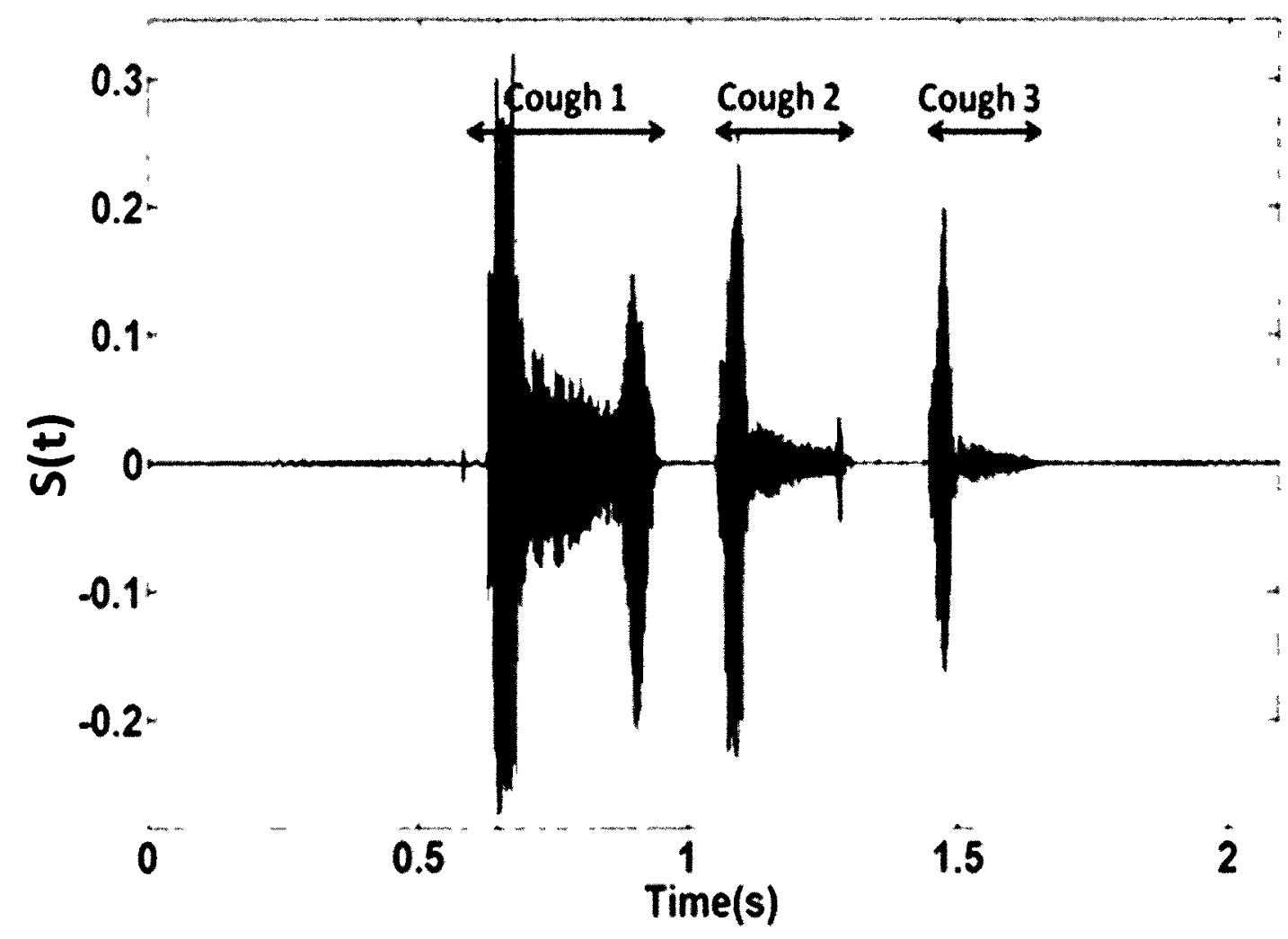

Figure 4.1: A typical cough sound recording. 
The first cough episode has a longer duration and more power than the subsequent cough episodes. Usually there is a gap between each cough episode and therefore it would be easy to distinguish each cough episode.

Since an automatic cough recording segmentation algorithm was out of the scope of this thesis, cough recording segmentation was performed manually. Each cough recording was divided into 3 or more individual cough signals. The final dataset contained nine highly dry and eight highly wet coughs and was used in this research.

\subsection{Pre-Processing Algorithm}

This section explains the pre-processing algorithm that was applied on each cough sound in the database. As mentioned in Section 4.1, the cough sound database was gathered from different sources. Hence, the recording methods and equipment were not identical among the cough samples. In order to ensure consistency, a pre-processing algorithm was applied to all the cough sounds as depicted in Figure 4.2.

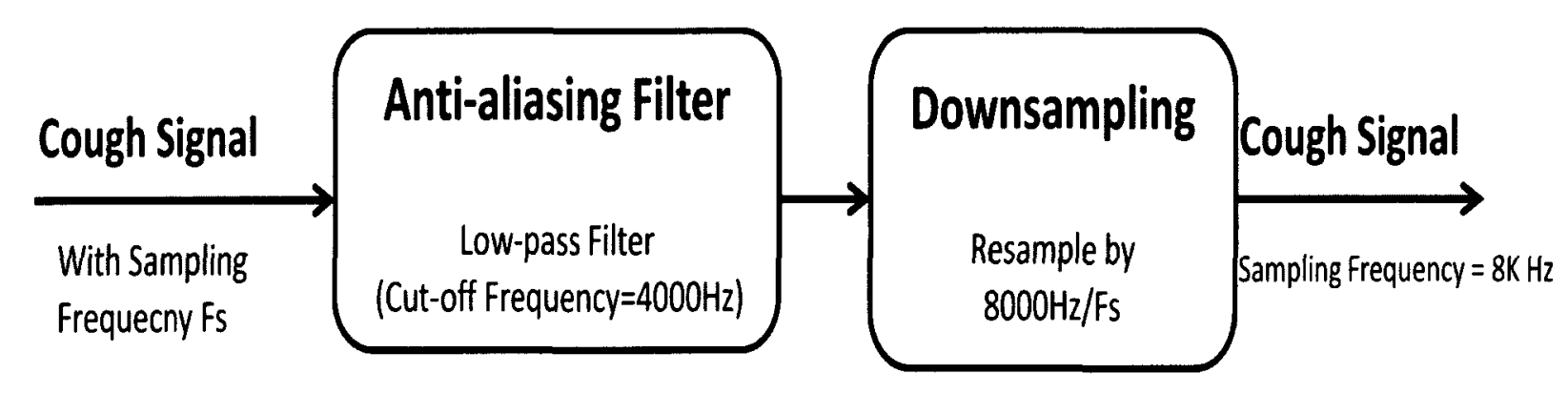

Figure 4.2: Pre-processing algorithm. 
The sampling frequency of each cough recording with the database was also different. In order to ensure a consistent sampling frequency, all the cough signals were resampled to $8 \mathrm{kHz}$. The resampling process was performed by passing the cough signal through an anti-aliasing filter with a cut-off frequency of $4 \mathrm{kHz}$ and resampling the cough signal to 8 $\mathrm{kHz}$ afterwards. It was observed that most of the frequency information of a cough sound was between $350 \mathrm{~Hz}$ and $4 \mathrm{kHz}$ [33]; therefore, by resampling the cough signals, important information was not being filtered out. 


\section{Chapter 5:}

\section{Feature Extraction Algorithm}

\subsection{Introduction}

This chapter provides two feature extraction algorithms in order to differentiate the dry and wet cough sounds. It begins by introducing a time domain feature in Section 5.2 and a frequency domain feature in Section 5.5. In each section the feature extraction algorithm is explained in details. Results obtained by using each feature are reported in Section 5.7 and are discussed in 5.8 .

\subsection{Time Domain Feature}

In Chapter 4, dry and wet cough sounds were analyzed in both time and frequency domains. Their characteristics in time and frequency domains were compared and their similarities and differences were explored. 
It was observed that dry cough signals have higher frequency components than wet cough signals. Wet cough signals tend to have more energy at lower frequencies than dry cough signals. To confirm this observation, both dry and wet cough signals were passed through a low-pass filter to filter out the higher frequency components. It was observed that wet cough signals contained higher energy and more amplitude variation after passing through the low-pass filter. Based on this observation, a band-pass filter was chosen to investigate more the characteristics of both types of cough at different frequency bands.

\subsection{Energy Envelope Variation Detection}

In this section, the algorithm for extracting a time domain feature, which is the variation of the energy envelope of the cough signal, will be explained and referred to as Feature 1. In order to extract Feature 1, the algorithm depicted in Figure 5.1 was used and is explained in the following sections in detail.

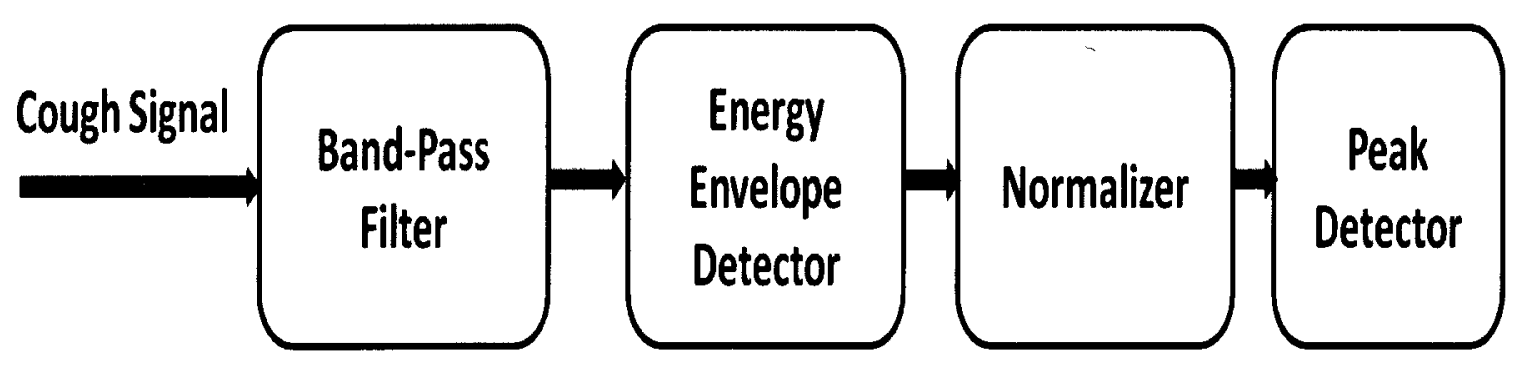

Figure 5.1: Feature 1 algorithm. 


\subsubsection{Band-Pass Filter}

The first step of extracting Feature 1 was to pass the cough signals through a band-pass filter. The band-pass filter was used in order to consider focused frequency bands and filter out the rest of the frequencies. A series of band-pass filters with frequency bands of $50 \mathrm{~Hz}$ between $0-1000 \mathrm{~Hz}$ were used (i.e., $0-50 \mathrm{~Hz}, 50-100 \mathrm{~Hz}, 100-150 \mathrm{~Hz}, \ldots$ 950-1000 $\mathrm{Hz}$ ). Magnitude response of two band-pass filters with cut-off frequencies of $200,250 \mathrm{~Hz}$ and $250,300 \mathrm{~Hz}$ are depicted in Figure 5.2.

a)

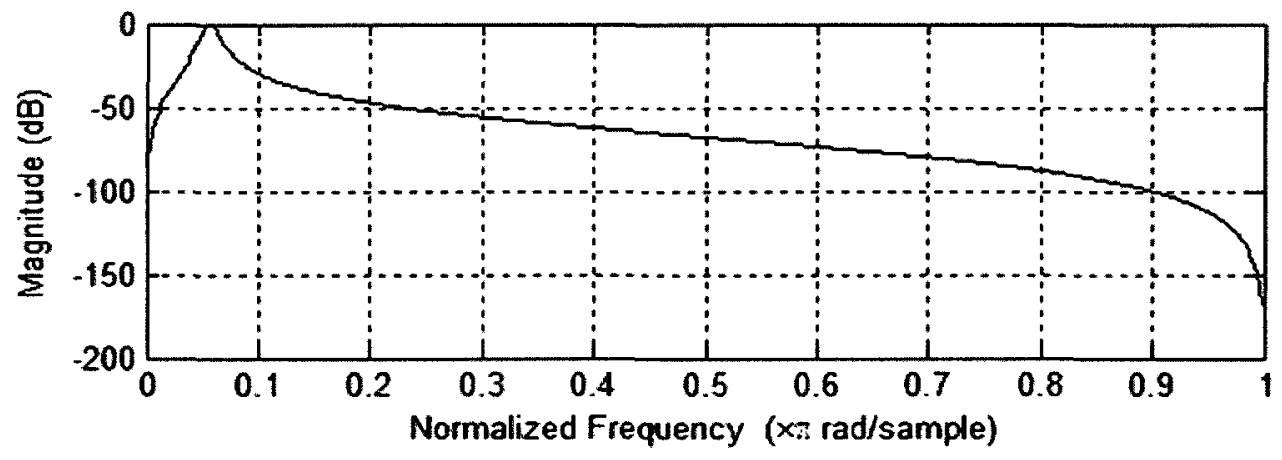

b)

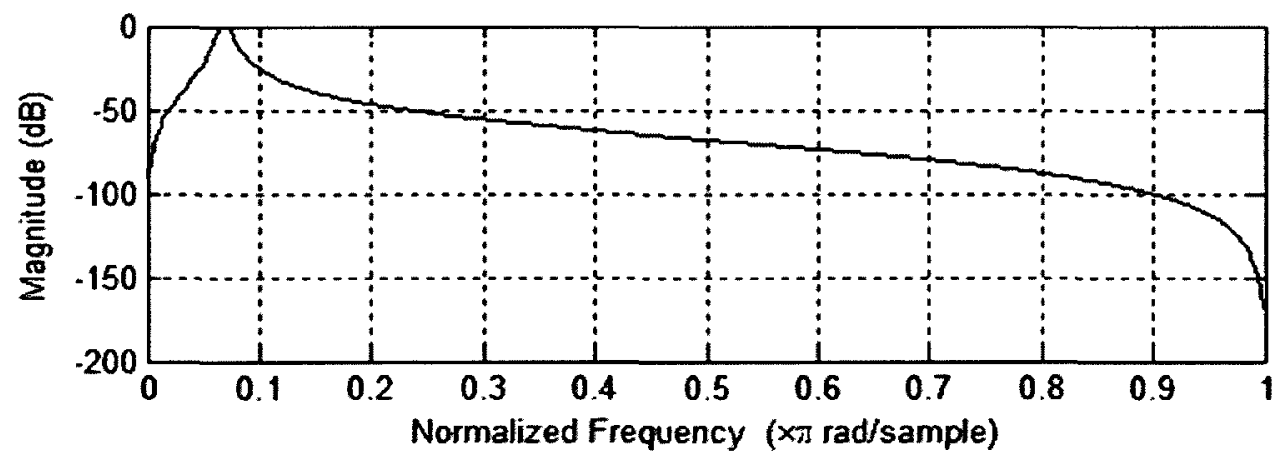

Figure 5.2: Magnitude response of a band-pass filter with cut-off frequencies of a) 200 and $250 \mathrm{~Hz}$. b) 250 and $300 \mathrm{~Hz}$. 
Figure 5.3 and Figure 5.4 show a dry cough and a wet cough signal along with their band-pass filtered signals at $200-250 \mathrm{~Hz}$ and $250-300 \mathrm{~Hz}$. Figure $5.3 \mathrm{~b}$ and Figure $5.3 \mathrm{c}$ show the band-pass filtered signal of the dry cough signal of Figure $5.3 \mathrm{a}$ at $200-250 \mathrm{~Hz}$ and 250-300 $\mathrm{Hz}$ respectively. As can been seen from the two band-pass filtered signals, the second phase of the dry cough signal does not contain much energy in these two frequency bands. This characteristic of the second phase is also true for other frequency bands, as was mentioned in the previous chapter. 
a)

b)

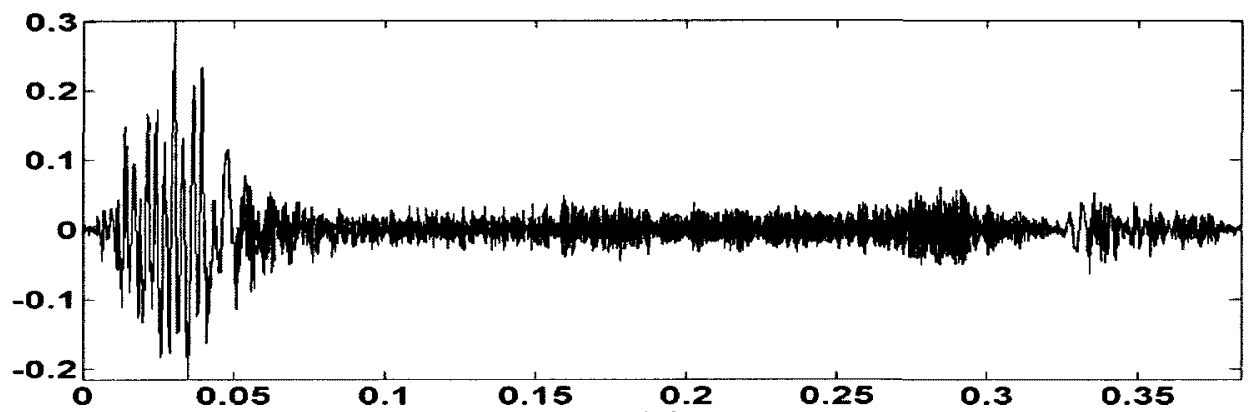

Time(s)

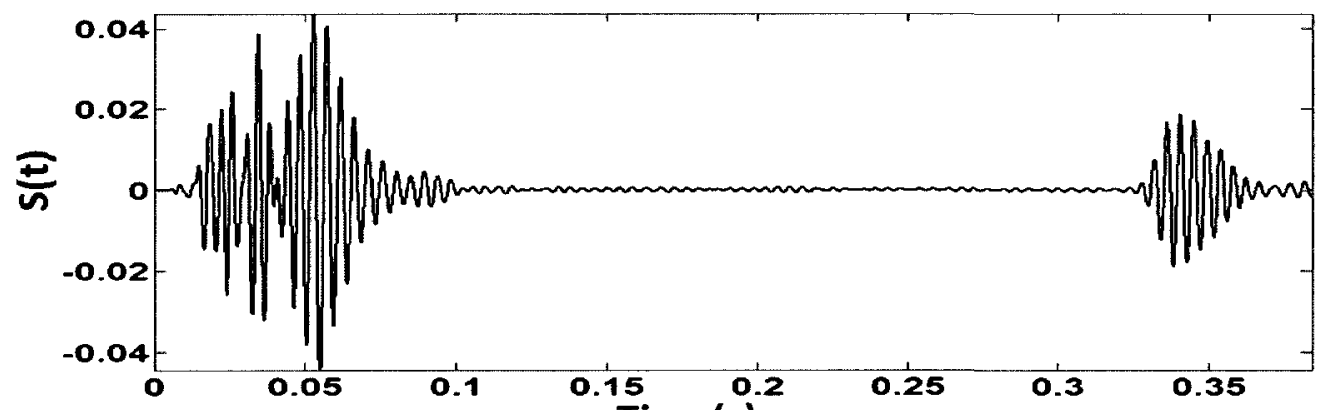

c)

Time(s)

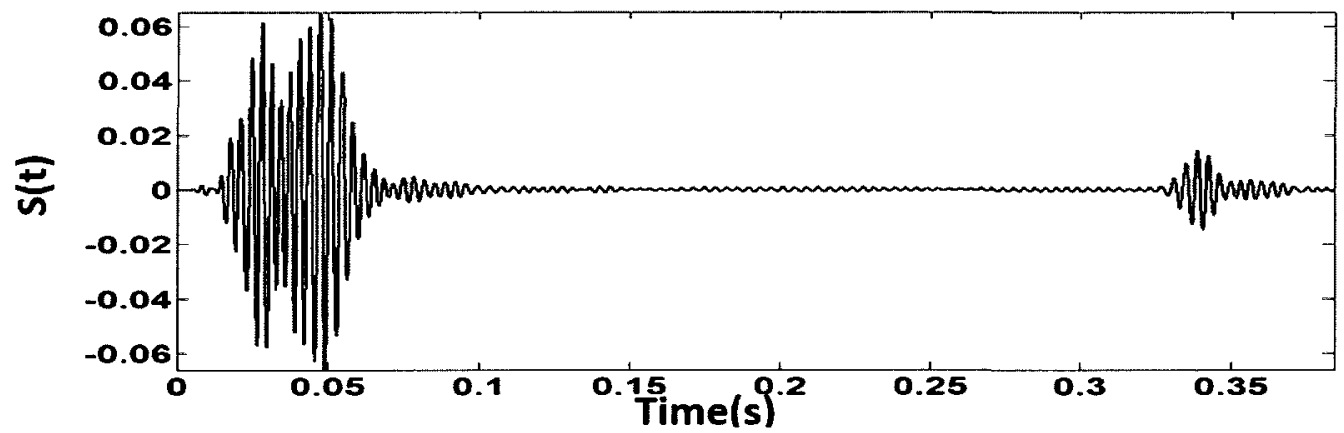

Figure 5.3: a) Dry cough signal. b) Band-pass filtered dry cough signal at 200-250 Hz. c)

Band-pass filtered dry cough signal at $250-300 \mathrm{~Hz}$.

On the other hand, after passing the wet cough signal through these two band-pass filters $(200-250 \mathrm{~Hz}$ and $250-300 \mathrm{~Hz})$, there is still a lot of the signal left at phase 2, as 
depicted in Figure 5.4b and Figure 5.4c. This observation is also true for other frequency bands.

a)

b)
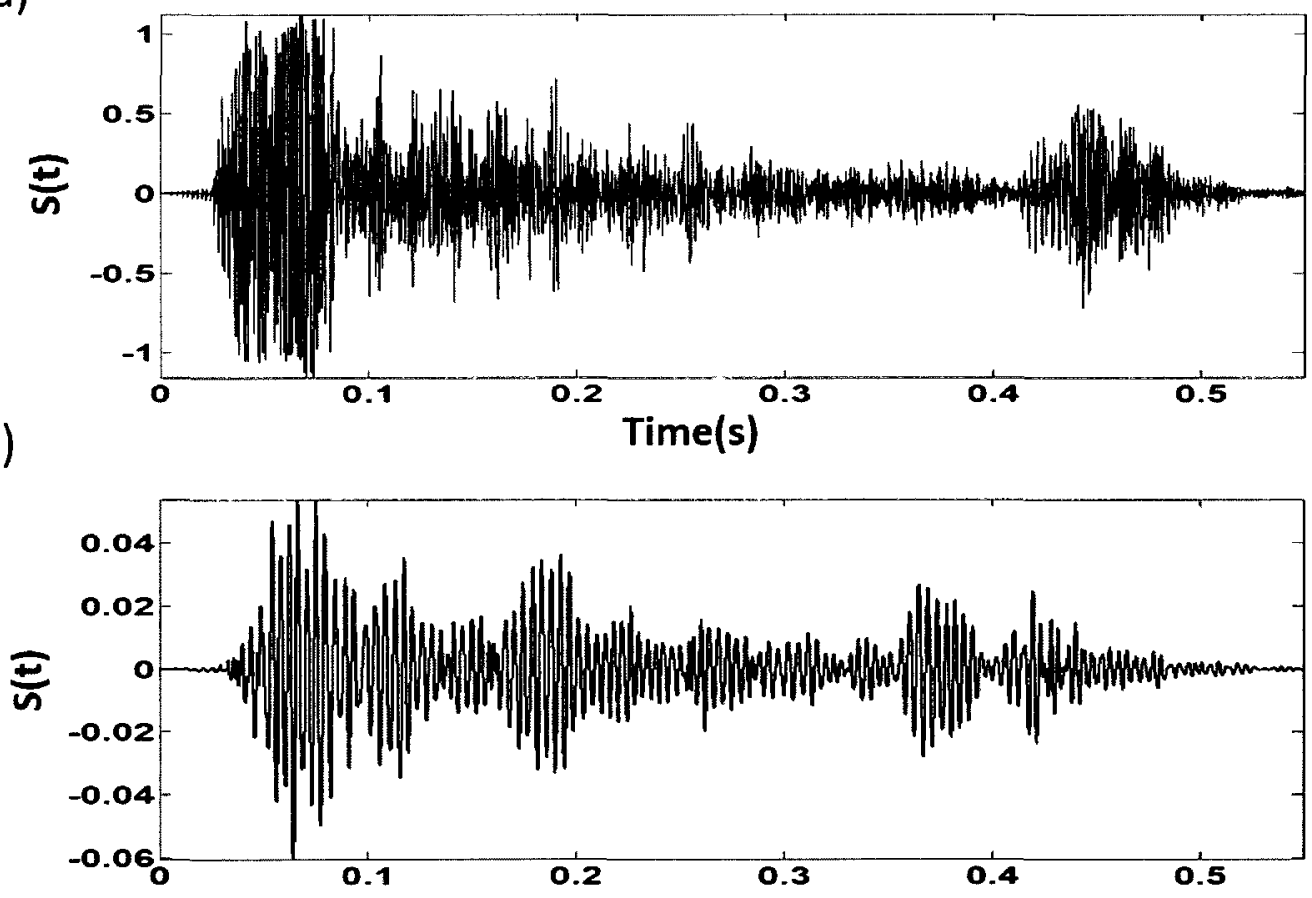

c)

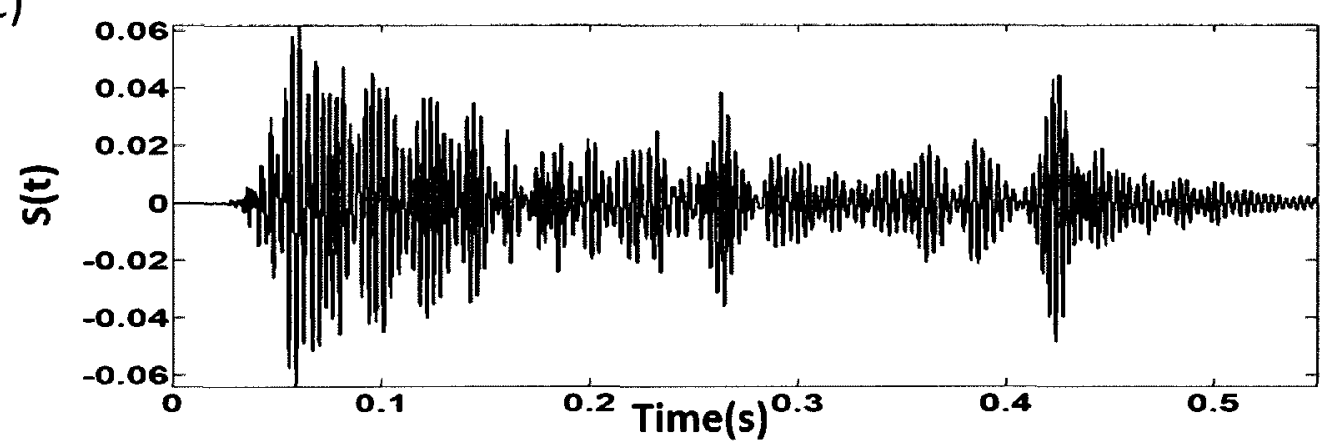

Figure 5.4: a) Wet cough signal. b) Band-pass filtered wet cough signal at 200-250 Hz. c) Band-pass filtered wet cough signal at $250-300 \mathrm{~Hz}$. 


\subsubsection{Energy Envelope Detector}

The energy envelope is determined by squaring the input signal $S(t)$ to obtain $S^{2}(t)$. Figure 5.5a and Figure 5.5b show the $\mathrm{S}^{2}(\mathrm{t})$ of the band-pass filtered dry cough signal from Figure 5.3b and Figure 5.3c.
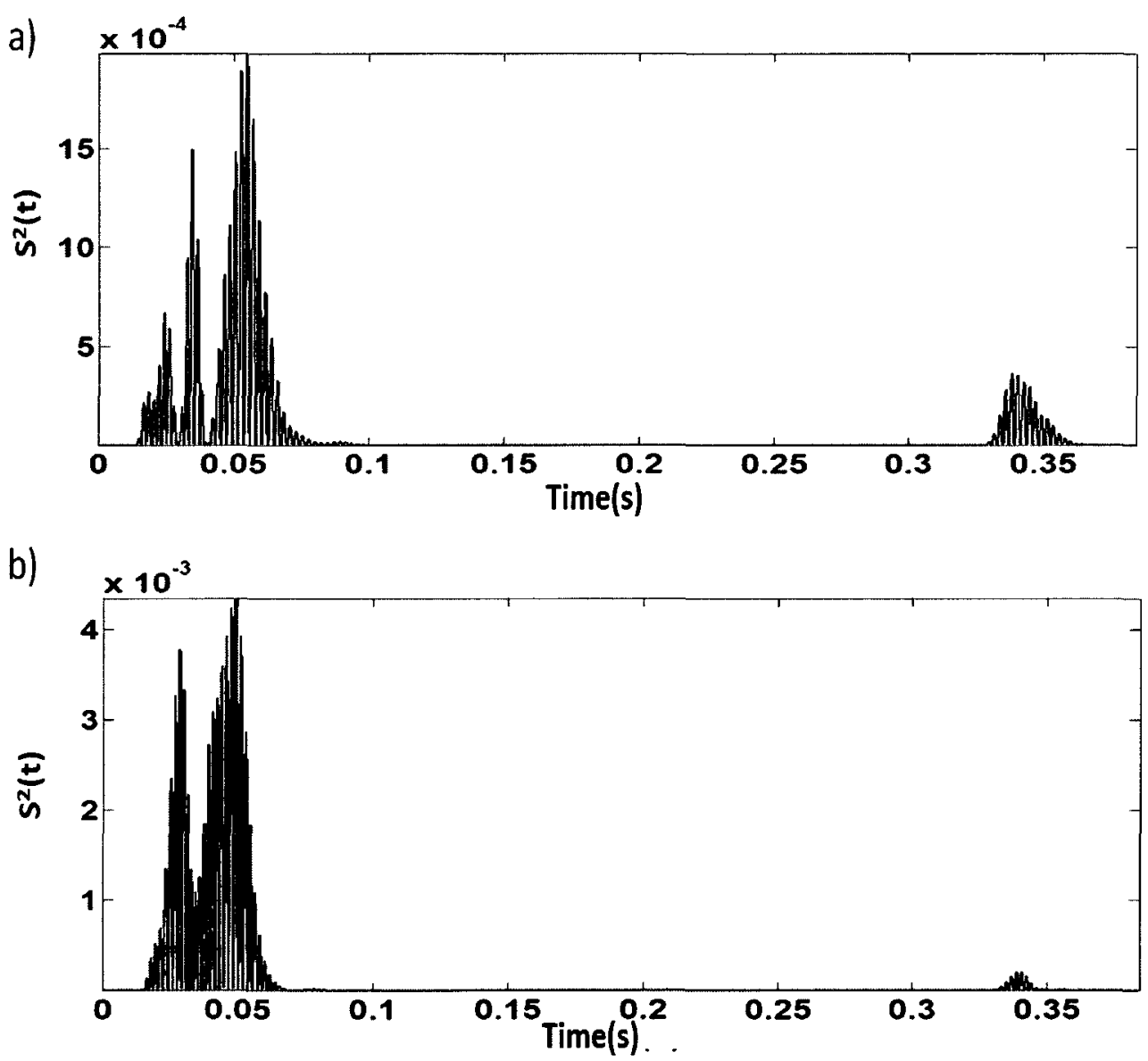

Figure 5.5: Signal square of a band-pass filtered dry cough signal at a) $200-250 \mathrm{~Hz}$, b) 250 $300 \mathrm{~Hz}$. 
Similarly, the $\mathrm{S}^{2}(\mathrm{t})$ of the two band-pass filtered wet cough signals (Figure $5.4 \mathrm{~b}$ and Figure 5.4c) are shown in Figure 5.6a and Figure 5.6b.
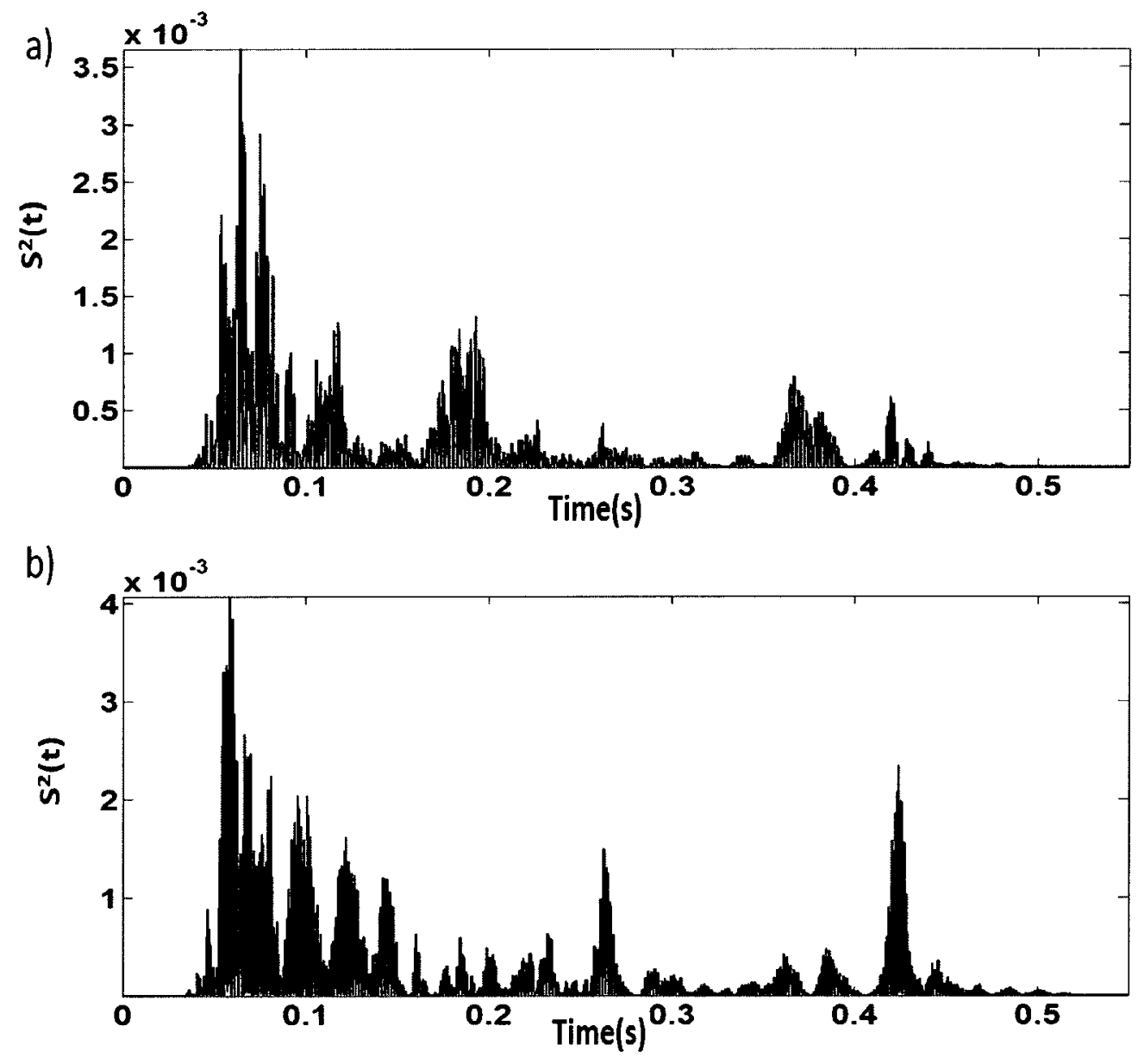

Figure 5.6: Signal square of a band-pass filtered wet cough signal at a) $200-250 \mathrm{~Hz}$, b) 250 $300 \mathrm{~Hz}$. 
After computing the square of the input signal, $S^{2}(t)$ was passed through a second order Butterworth low-pass filter with a cut-off frequency of $10 \mathrm{~Hz}$ (depicted in Figure 5.7) to determine its energy envelope $E(n)$. The cut-off frequency of the low-pass filter was chosen experimentally.

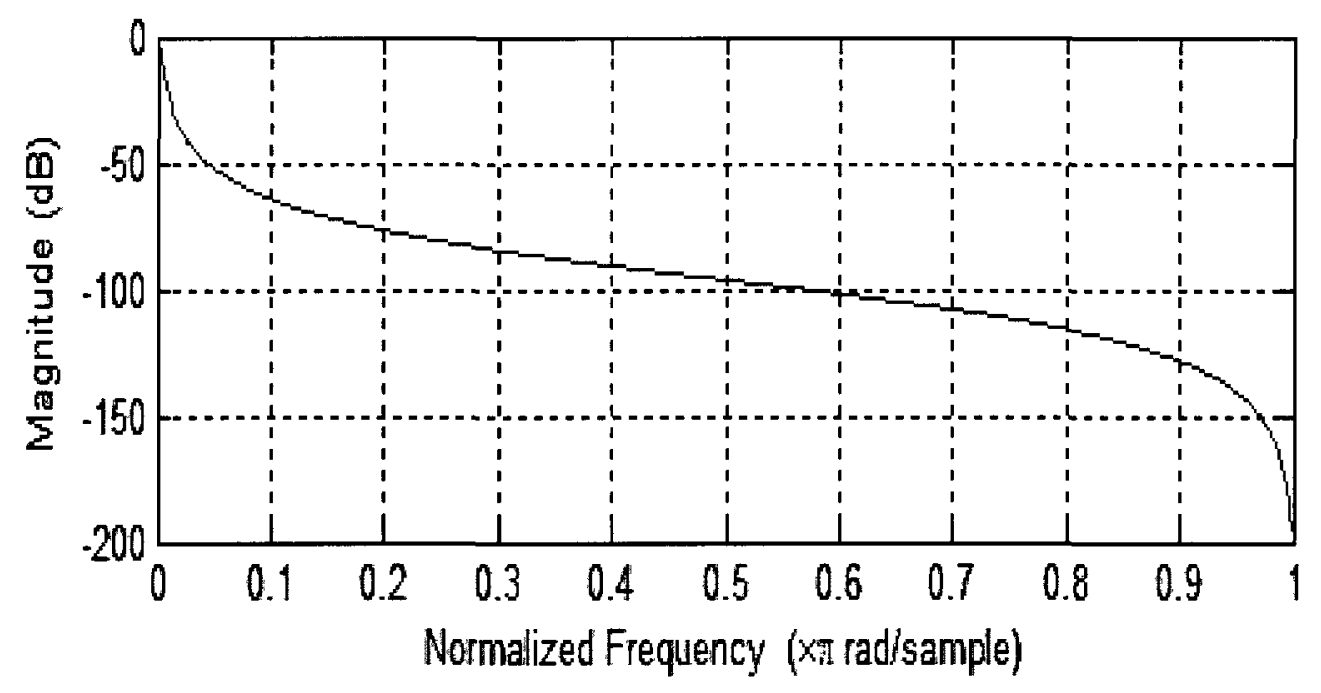

Figure 5.7: Magnitude response of a low-pass filter with cut-off frequency of $10 \mathrm{~Hz}$.

The energy envelope of both dry and wet cough signals for two frequency bands 200$250 \mathrm{~Hz}$ and 250 and $300 \mathrm{~Hz}$ are shown in Figure 5.8 and Figure 5.9 respectively. 

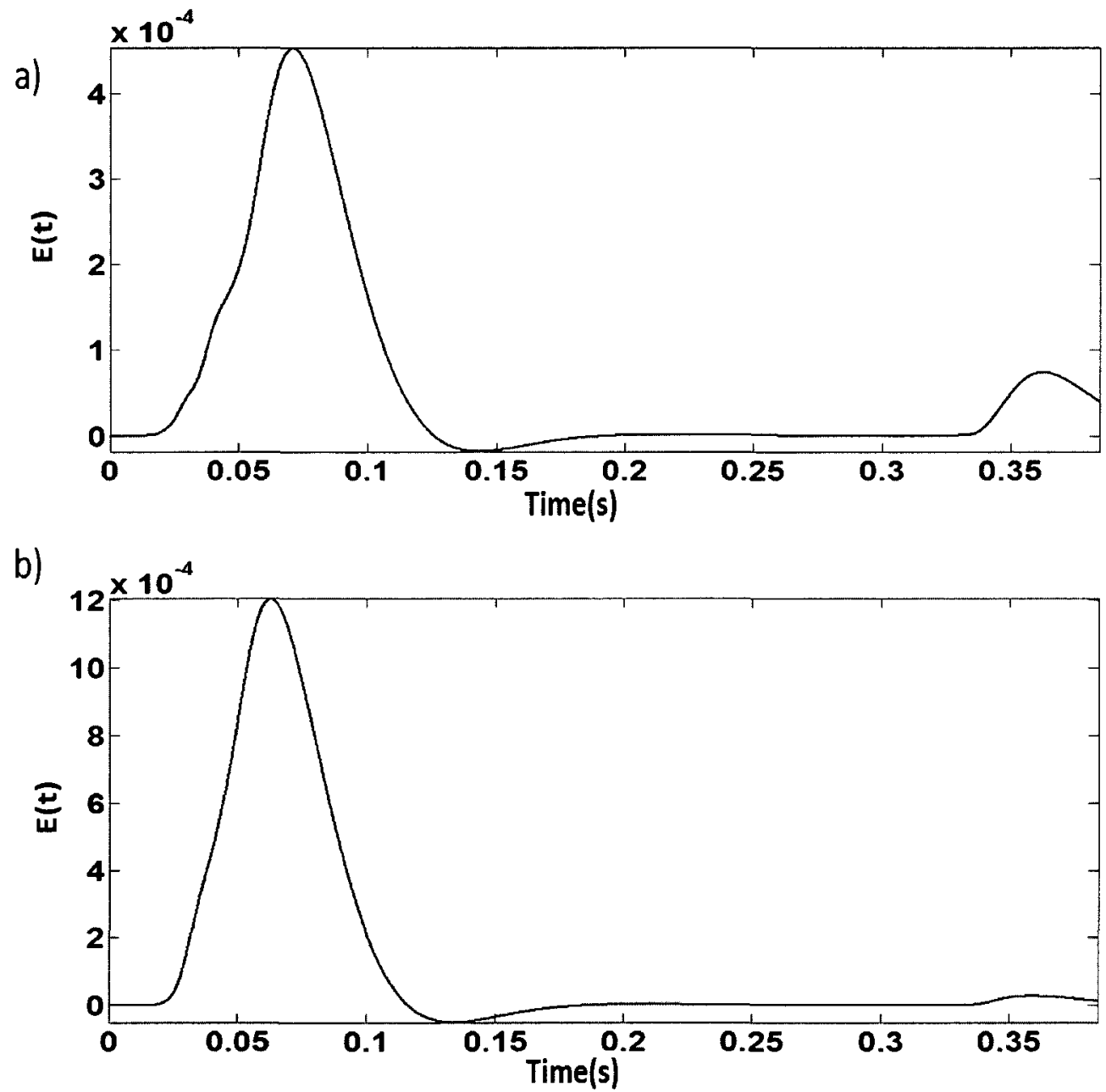

Figure 5.8: Energy envelope of dry cough signal at a) $200-250 \mathrm{~Hz}$ b) $250-300 \mathrm{~Hz}$. 
a)

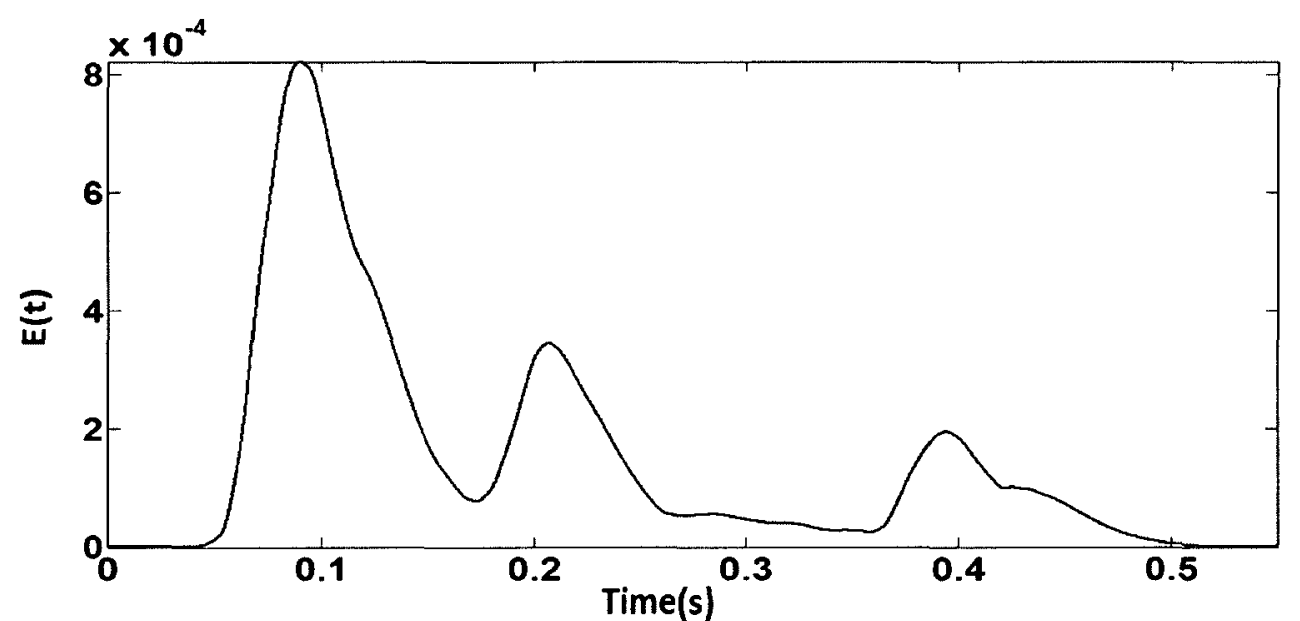

b)

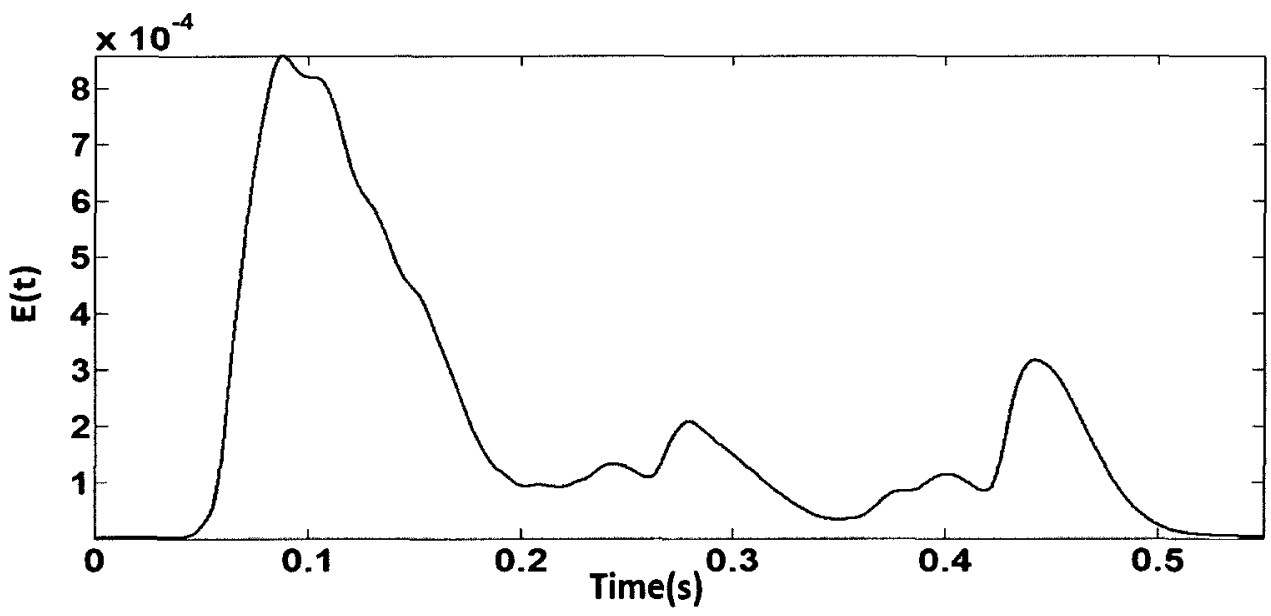

Figure 5.9: Energy envelope of wet cough signal at a) $200-250 \mathrm{~Hz}$ b) $250-300 \mathrm{~Hz}$.

\subsubsection{Energy Envelope Normalizer}

The next step was to normalize the energy envelope of each cough signal between 0 and 1. The reason for normalization was to compensate for the recording amplitude differences between the cough recordings. 
Normalized energy envelope of a dry and a wet cough signal at $200-250 \mathrm{~Hz}$ and 250 $300 \mathrm{~Hz}$ are depicted in Figure 5.10 and Figure 5.11 respectively. Comparing the energy envelope of dry and wet cough signals shows that there is more variation in shape for wet cough signals in Phase 2 than there is for dry cough signals. There are some similarities, however, between dry and wet cough signal energy envelope E(n). E(n) of the dry cough signals tend to be flat in Phase 2. Energy envelope of the wet cough signals however, contradicts this observation. The E(n) of wet cough signals has more variation, especially in Phase 2. 

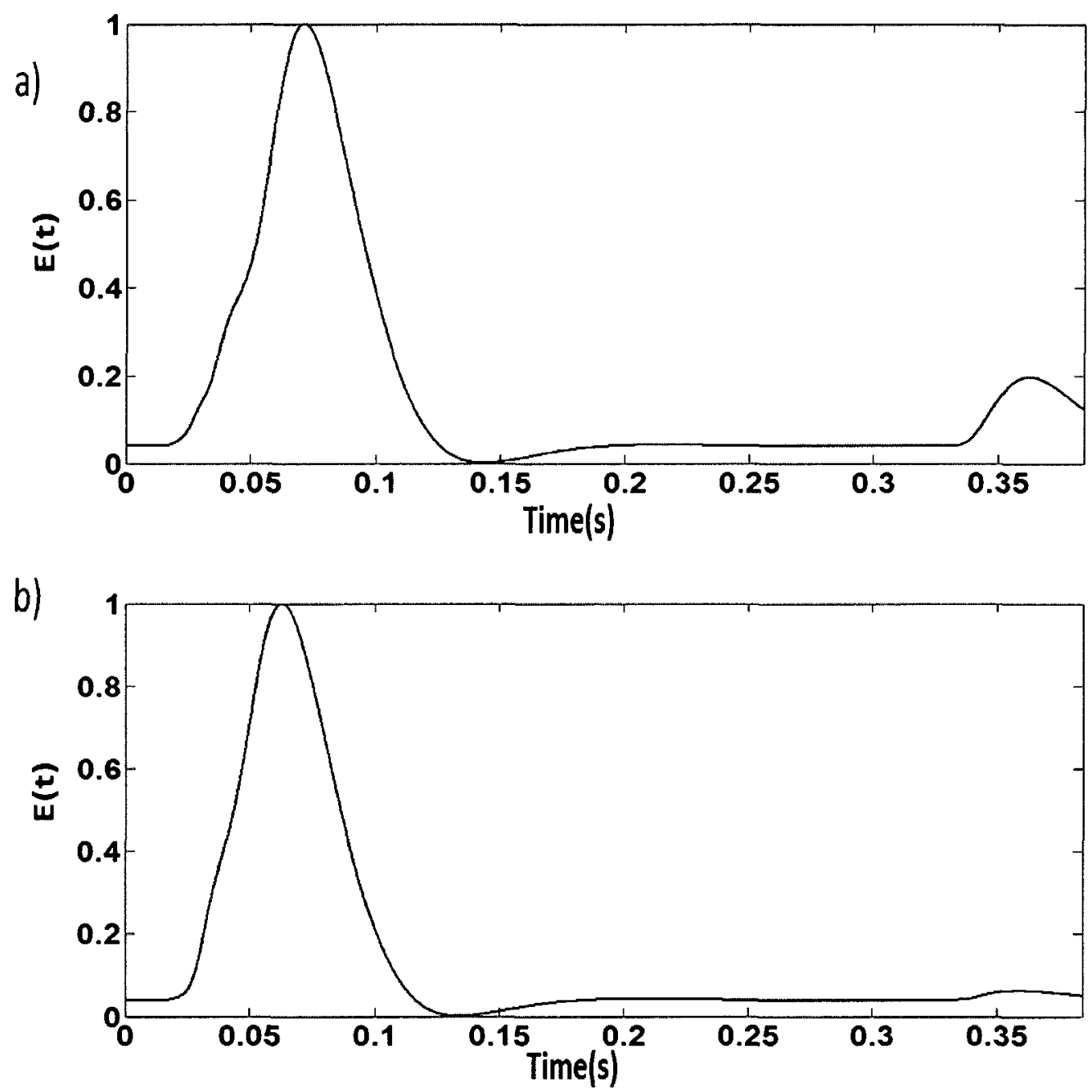

Figure 5.10: Normalized energy envelope of a dry cough signal at a) 200-250 Hz, b) 250-300 Hz. 
a)

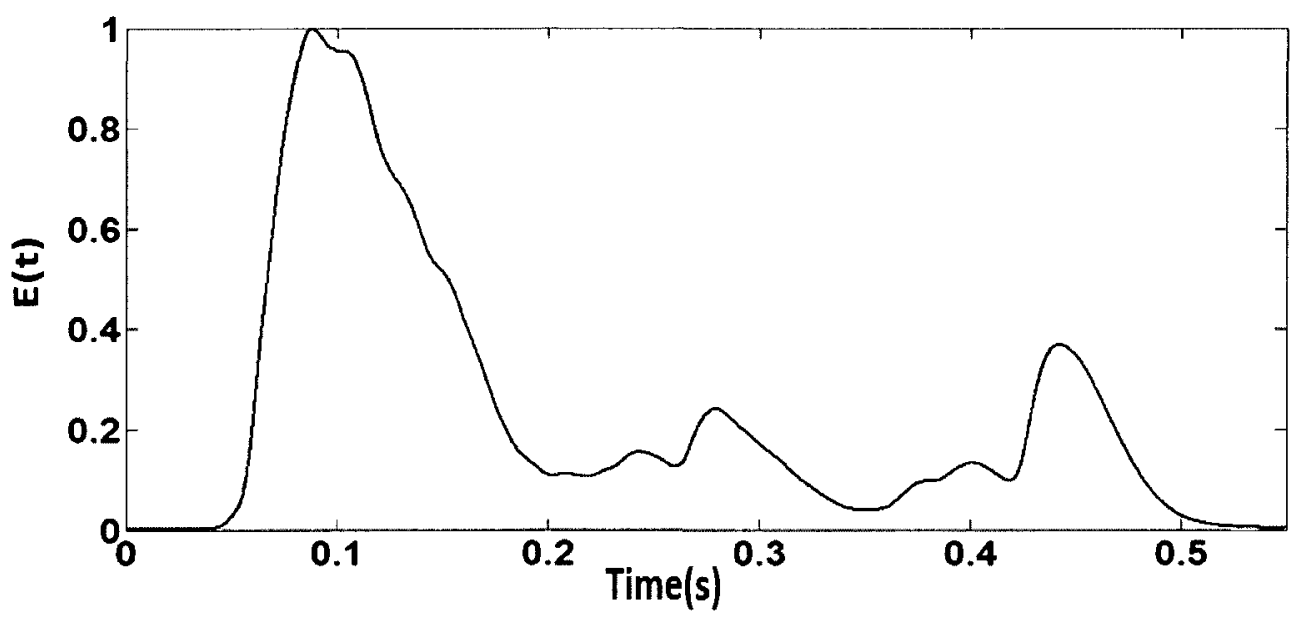

b)

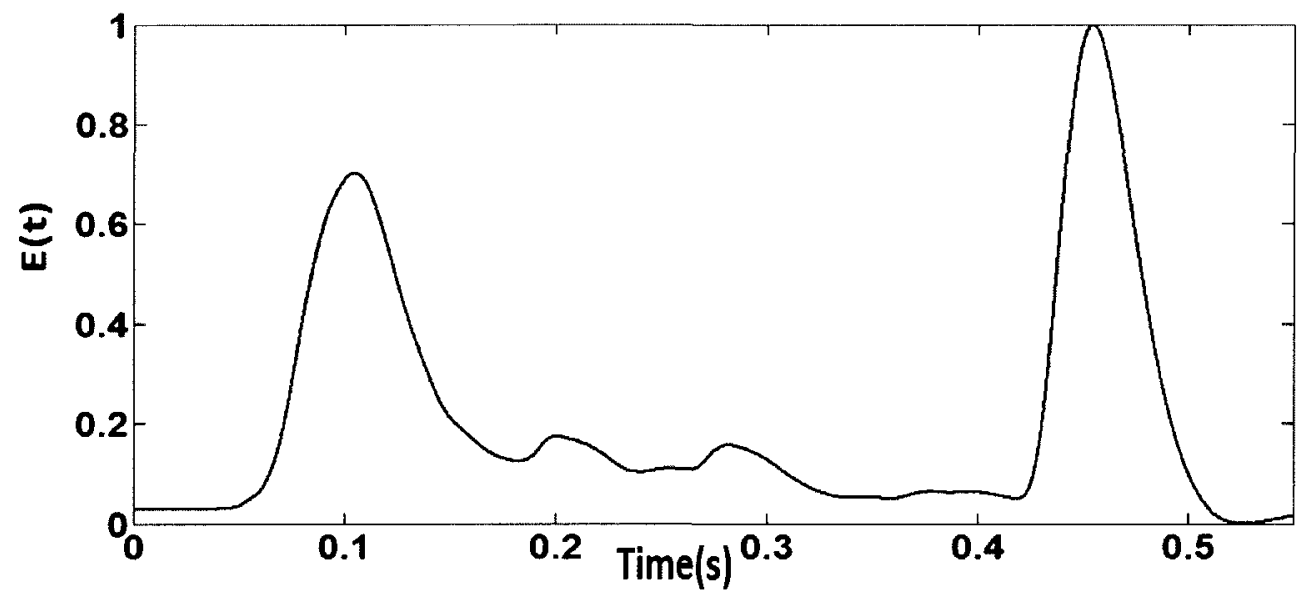

Figure 5.11: Normalized energy envelope of a wet cough signal at a) $200-250 \mathrm{~Hz}$, b) $250-300$ Hz.

\subsubsection{Peak Detector}

As stated in the previous section, there is a significant shape difference between the energy envelope of the two types of cough signals. In order to use this difference as a descriptive feature, a peak detection algorithm was used and is depicted in Figure 5.12. 
The peak detection algorithm compares two adjacent points to find either the local maxima or a flat region. If a flat region is detected, it skips one or two points depending on the region being a 'short' or 'long' flat region respectively. If a local maximum greater than a certain threshold is found, it will be marked as a significant peak. The threshold was chosen to be 0.05 experimentally.

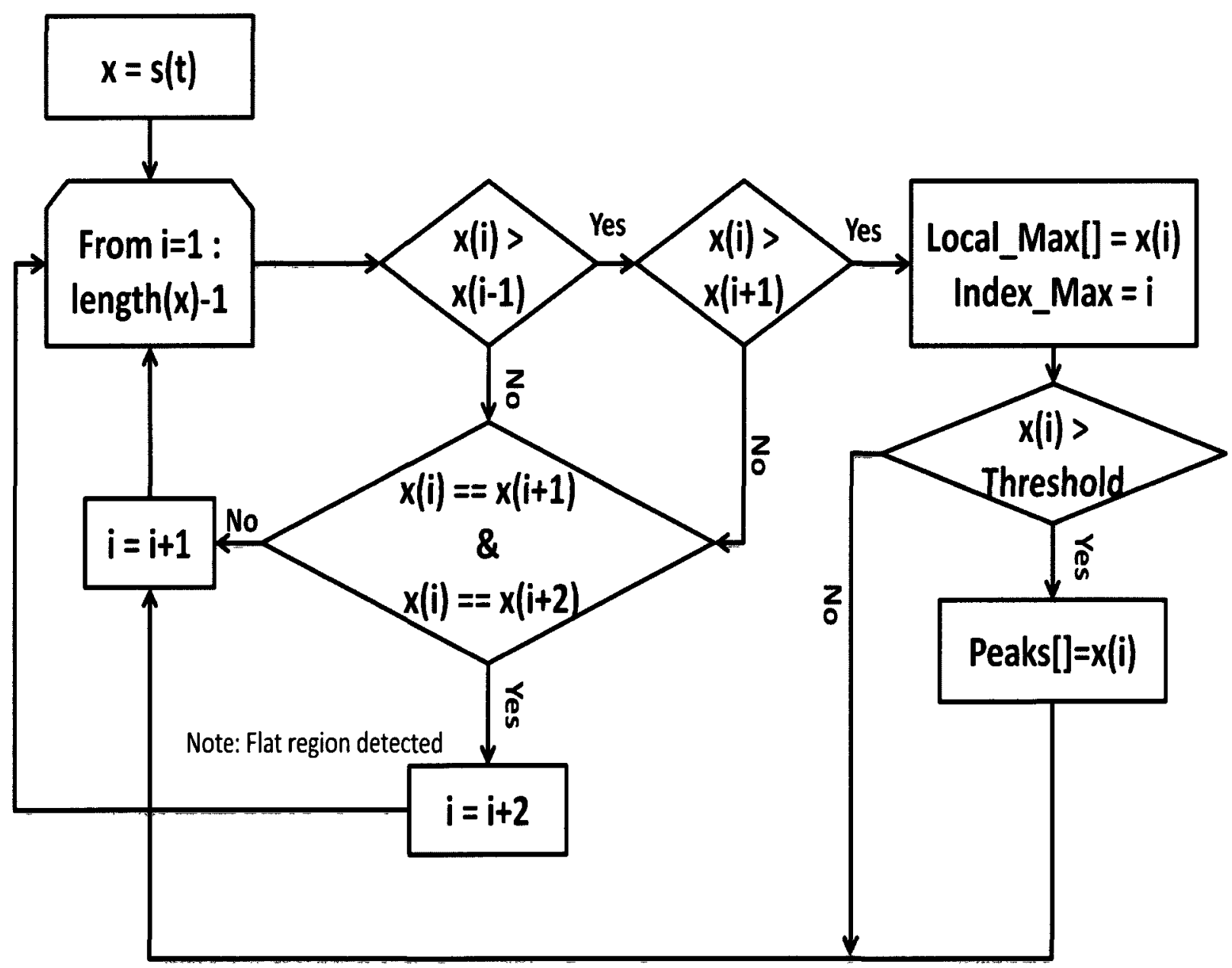

Figure 5.12: Peak Detector Algorithm. 
Peaks of the energy envelope of dry and wet cough signals were detected using the explained peak detector algorithm. As an example, peaks of the energy envelope of two dry and two wet cough signals at $200-250 \mathrm{~Hz}$ are shown in Figure 5.13 .

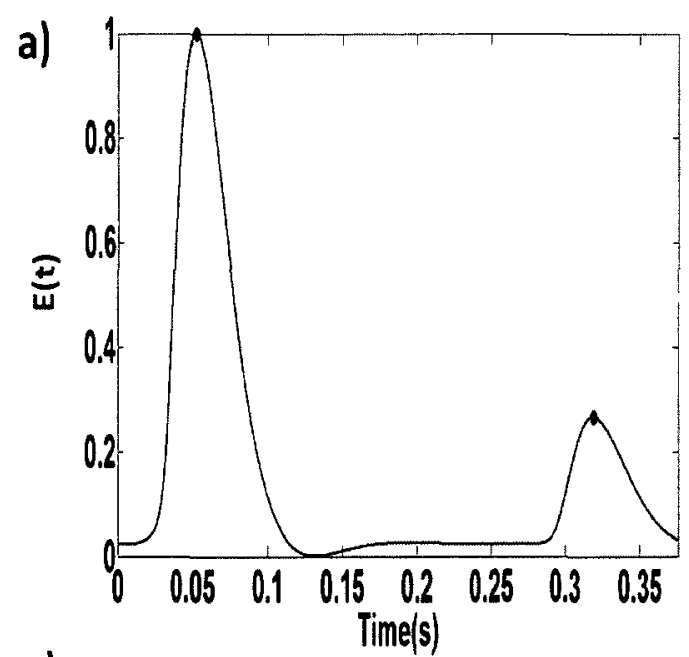

c)

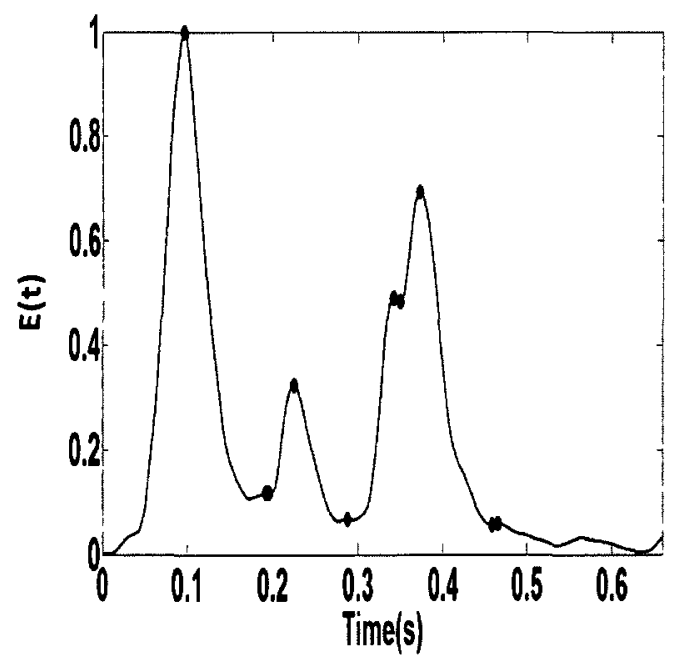

b)

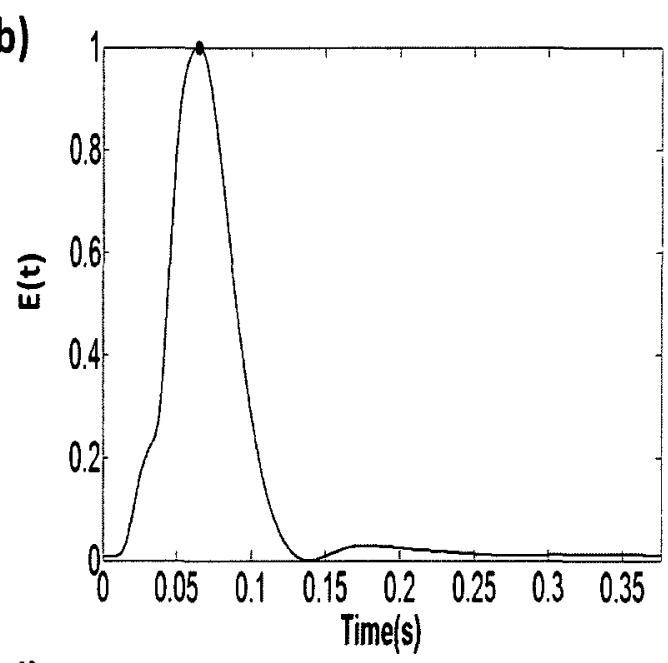

d)

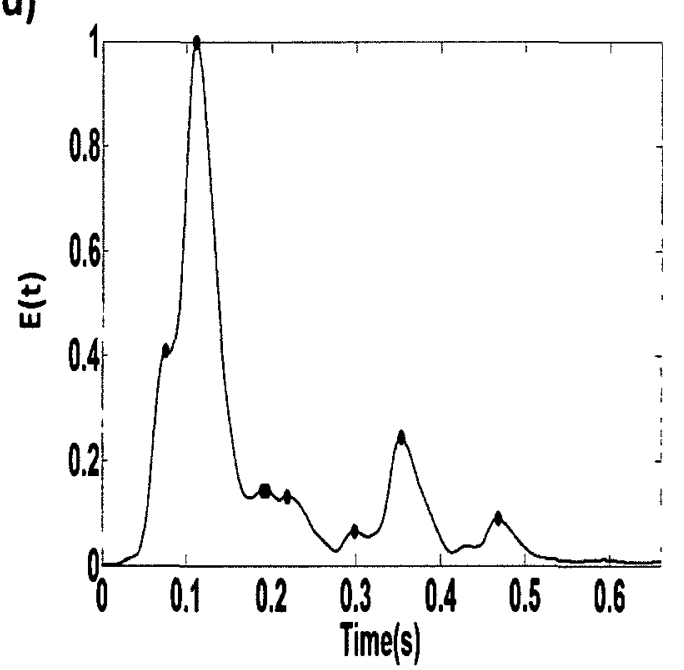

Figure 5.13: Normalized energy envelope and peaks of a) Dry cough sample 1, b) Dry cough sample 2 , c) Wet cough sample 1 , and d) Wet cough sample 2. 


\subsubsection{Energy Envelope Characteristic Differences}

It was observed that the energy envelope of the dry cough signal followed a specific shape. It started with a peak, which represented the first phase of the dry cough signal followed by a flat region representing the second phase. It finished with a small peak, if a third phase existed for that particular signal, as depicted in Figure 5.14a. The energy of the wet cough signal, on the other hand, did not follow a specific shape. The shape was more random and multiple peaks were observed especially in the second phase, as depicted in Figure 5.14b.

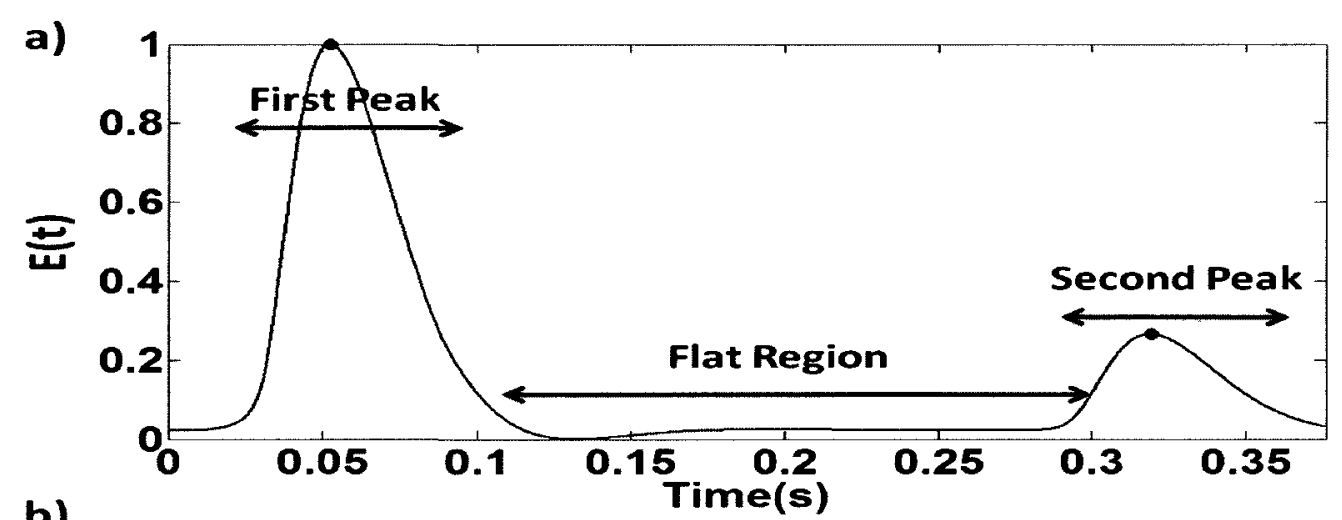

b)

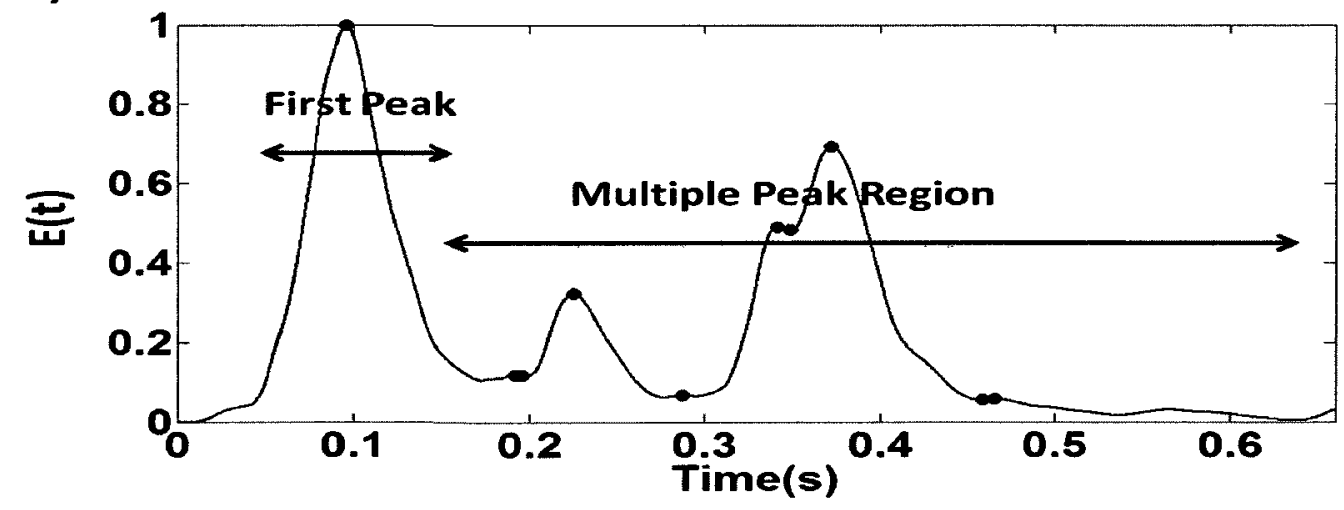

Figure 5.14: a) Energy envelope of a dry cough, and b) Energy envelope of a wet cough. 


\subsection{The Impact of Low-pass Filter}

In Section 5.3, the impact of a band-pass filter was analyzed on both dry and wet cough signals. In this section, the impact of a low-pass filter on dry and wet cough signals will be discussed and summarized in Sections 5.4.1 and 5.4.2.

\subsubsection{Dry Cough Signal}

The following figures show the process of extracting Feature 1 for dry coughs using a low-pass filter (LPF). A dry cough sample is shown in Figure 5.15 and its low-pass filtered signals at different cut-off frequencies are shown in Figure 5.16, Figure 5.17, Figure 5.18, Figure 5.19 and Figure 5.20. Furthermore, the squares of the low-pass filtered dry cough signals, $\mathrm{S}^{2}(\mathrm{t})$, are shown in Figure 5.21, Figure 5.22, Figure 5.23, Figure 5.24, and Figure 5.25.

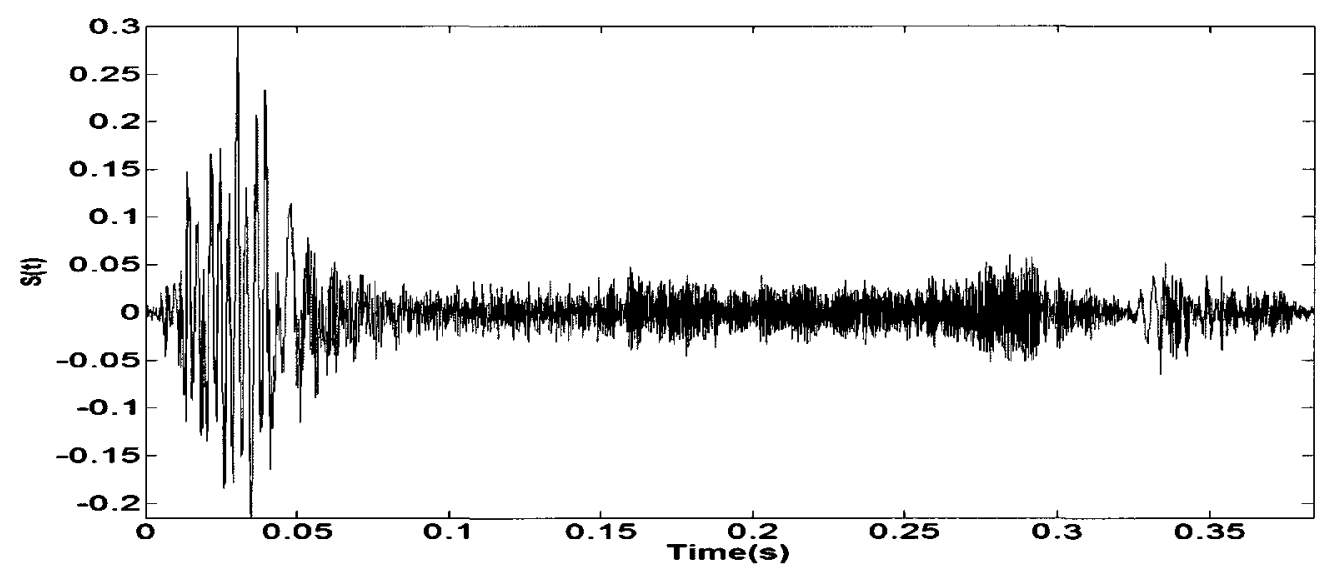

Figure 5.15: Dry cough sample. 


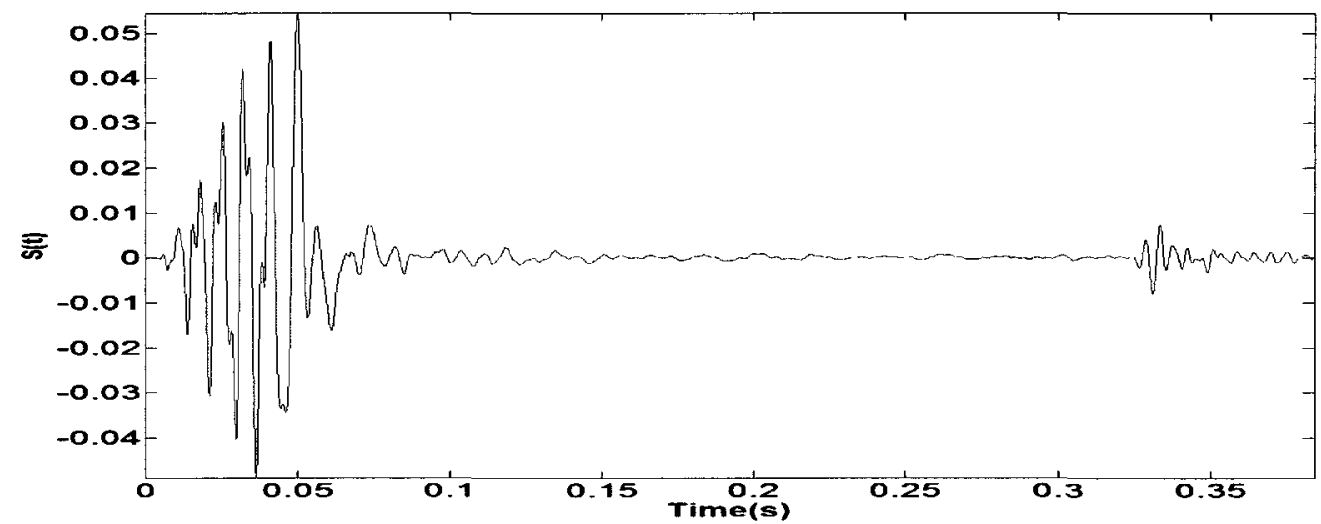

Figure 5.16: Low-pass filtered dry cough sample at $100 \mathrm{~Hz}$.

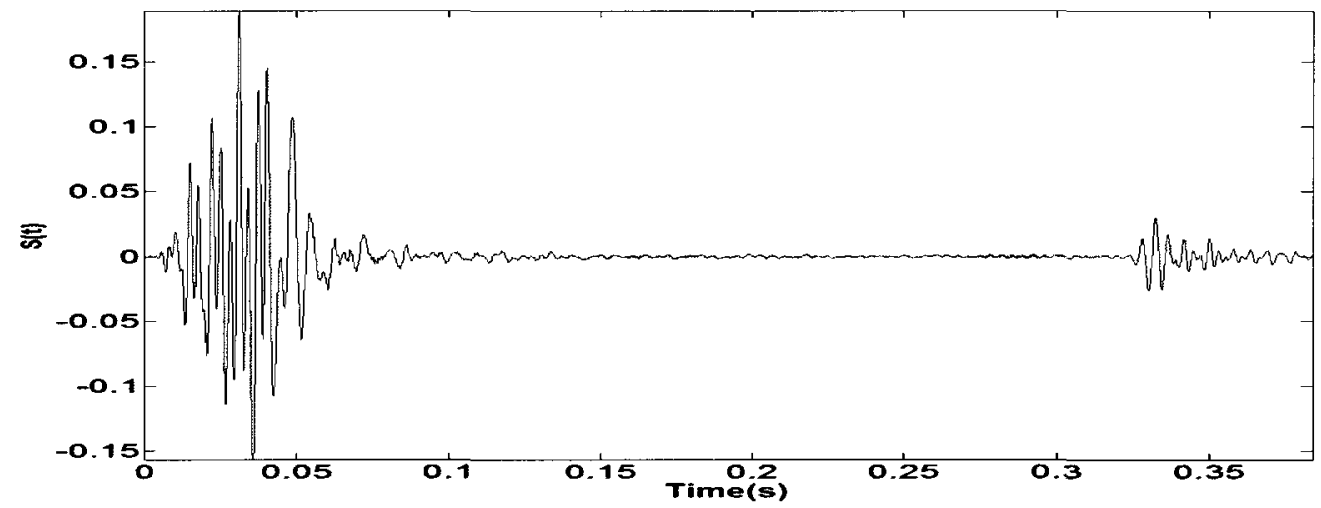

Figure 5.17: Low-pass filtered dry cough sample at $300 \mathrm{~Hz}$.

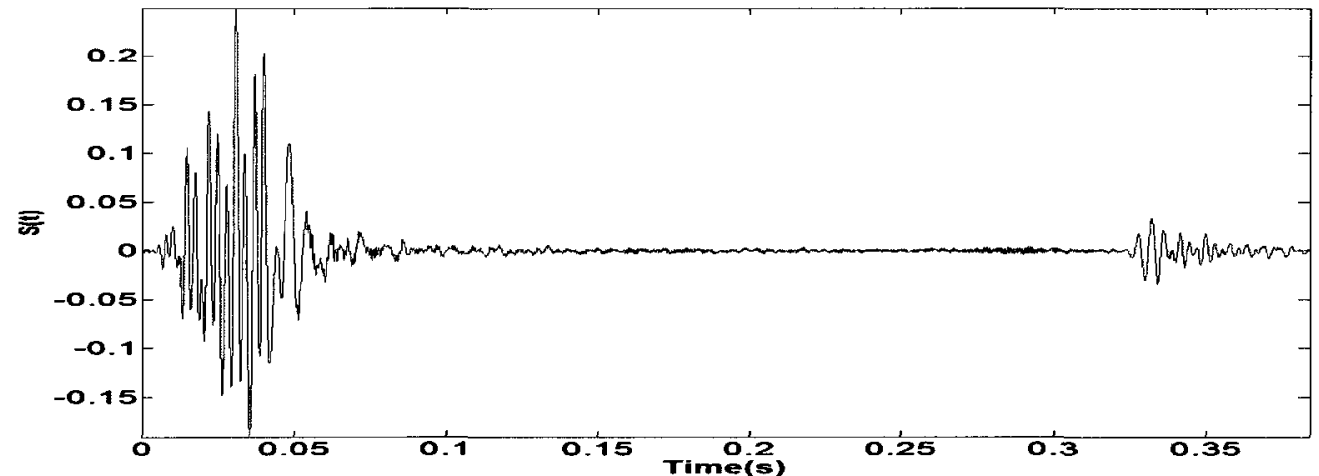

Figure 5.18: Low-pass filtered dry cough sample at $500 \mathrm{~Hz}$. 


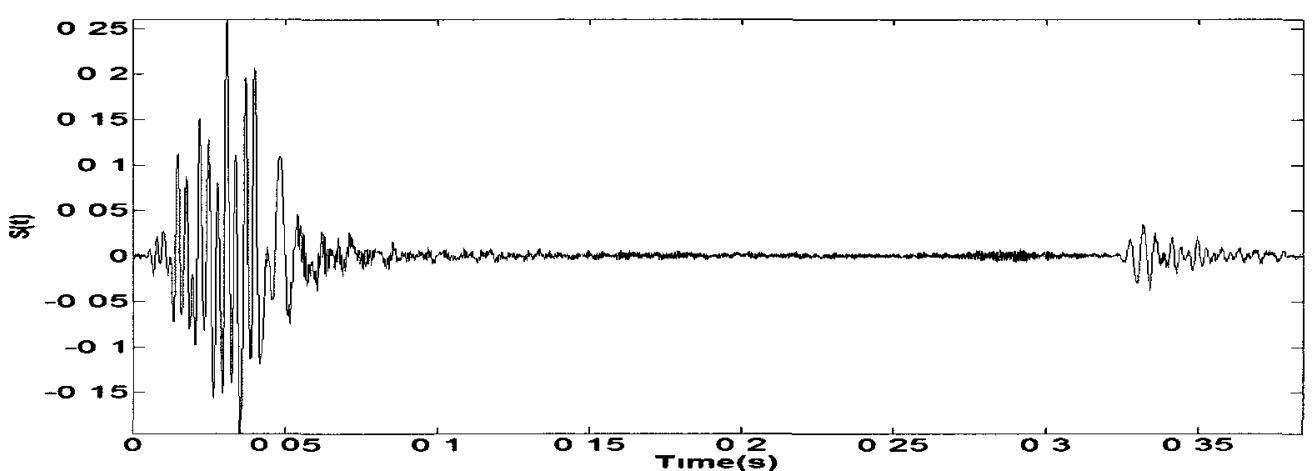

Figure 5.19: Low-pass filtered dry cough sample at $700 \mathrm{~Hz}$.

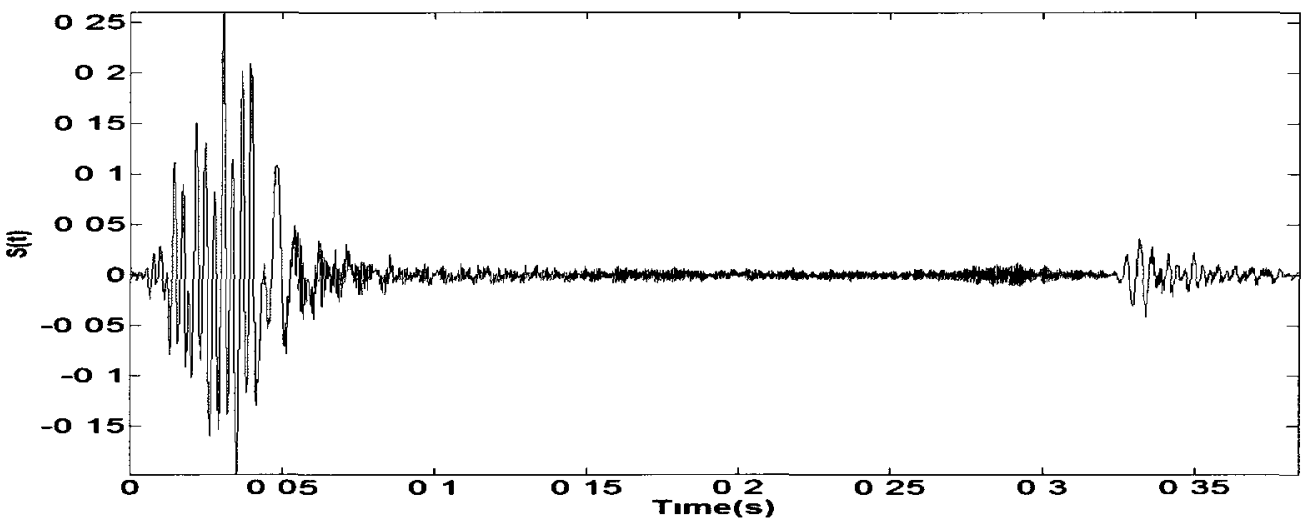

Figure 5.20: Low-pass filtered dry cough sample at $900 \mathrm{~Hz}$. 


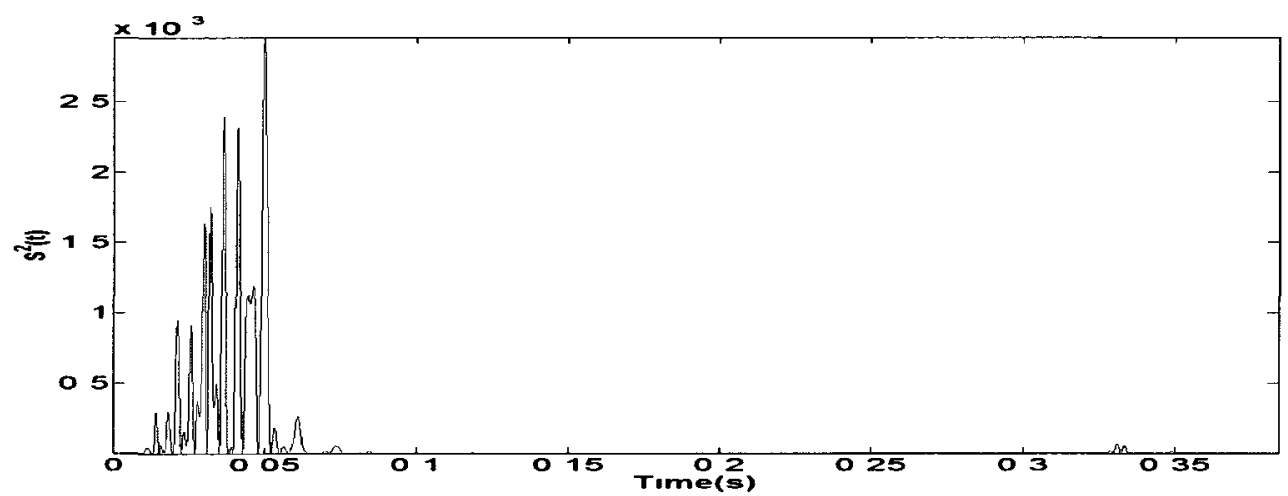

Figure 5.21: Signal squared of a low-pass filtered dry cough sound at $100 \mathrm{~Hz}$.

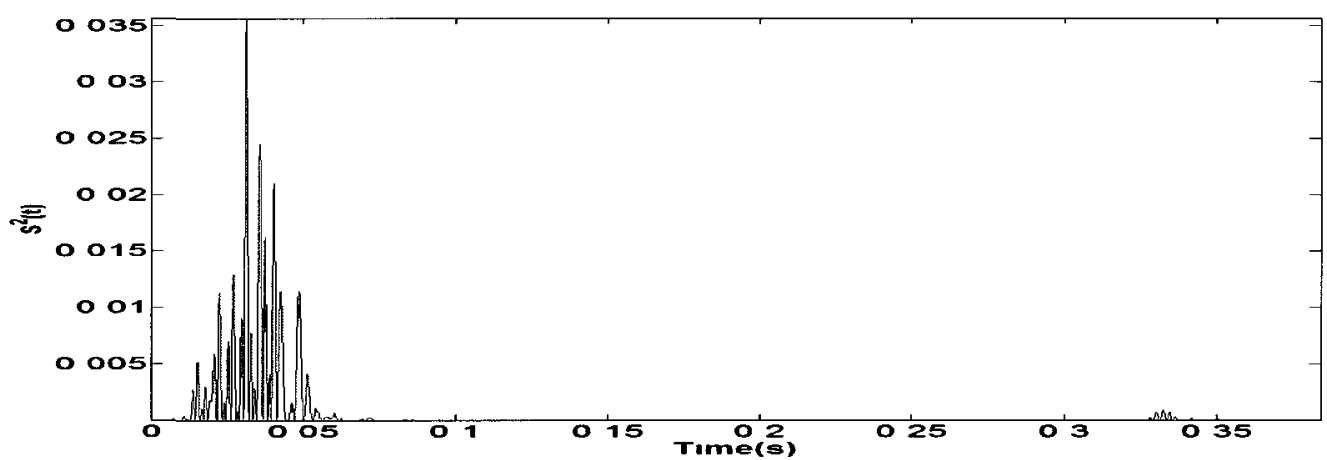

Figure 5.22: Signal squared of a low-pass filtered dry cough sound at $300 \mathrm{~Hz}$.

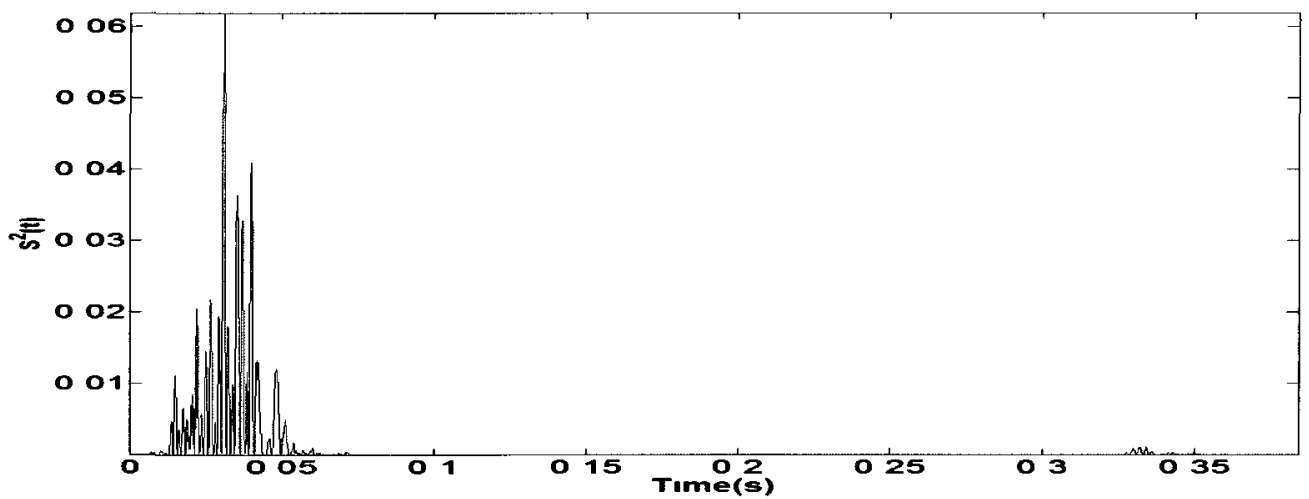

Figure 5.23: Signal squared of a low-pass filtered dry cough sound at $500 \mathrm{~Hz}$. 


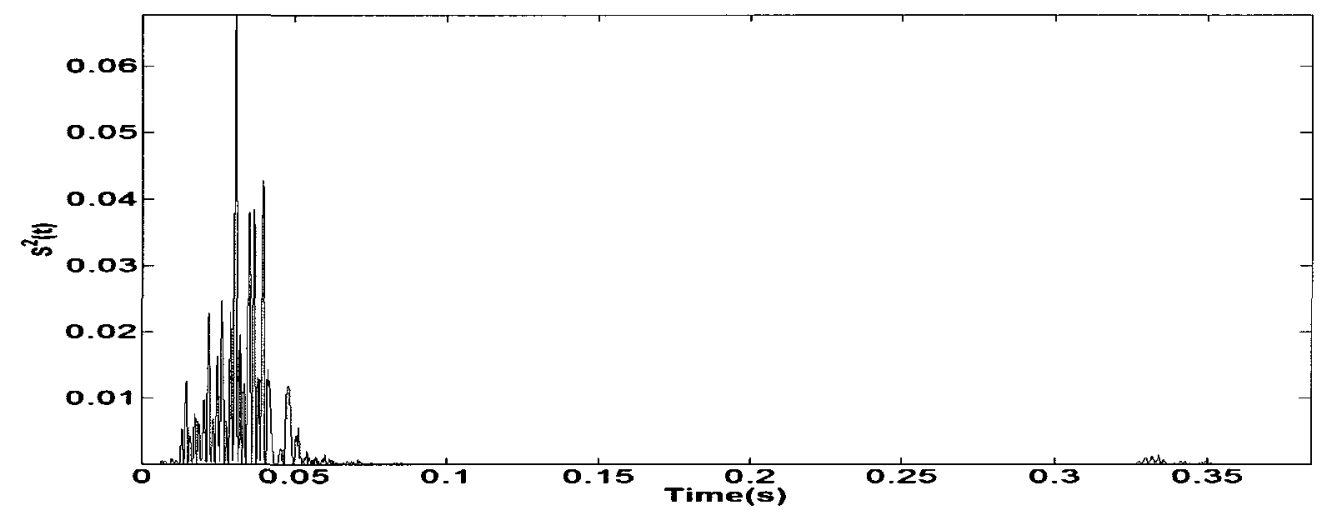

Figure 5.24: Signal squared of a low-pass filtered dry cough sound at $700 \mathrm{~Hz}$.

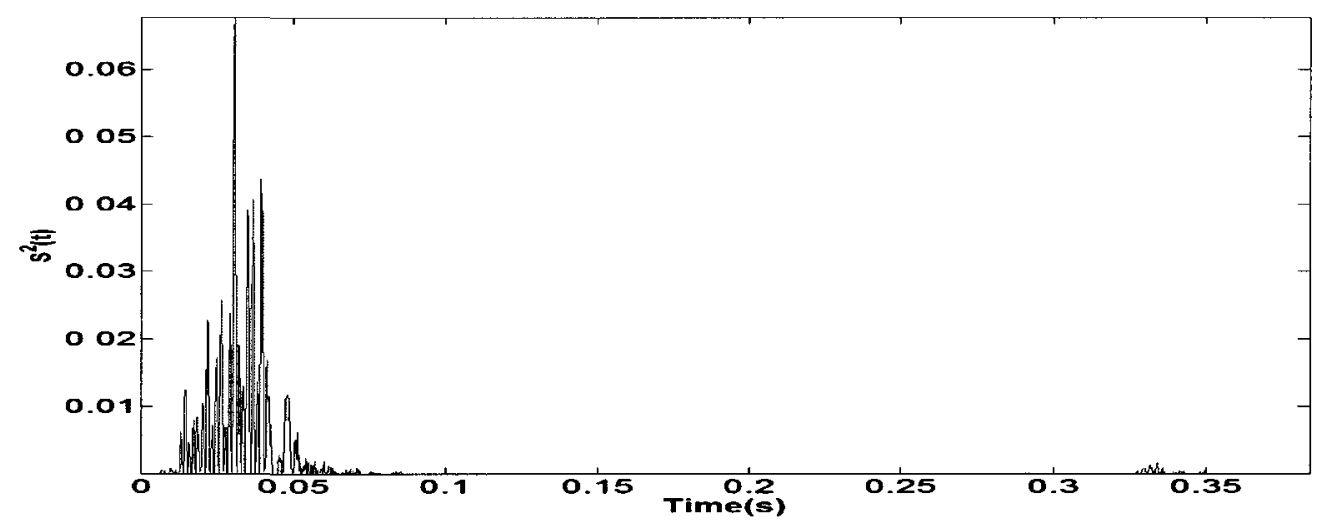

Figure 5.25: Signal squared of a low-pass filtered dry cough sound at $900 \mathrm{~Hz}$.

Finally, the energy envelope, $E(t)$, and peaks of the $S^{2}(t)$ of dry cough signals at different frequency bands are computed and depicted in Figure 5.26, Figure 5.27, Figure 5.28, Figure 5.29, and Figure 5.30. 


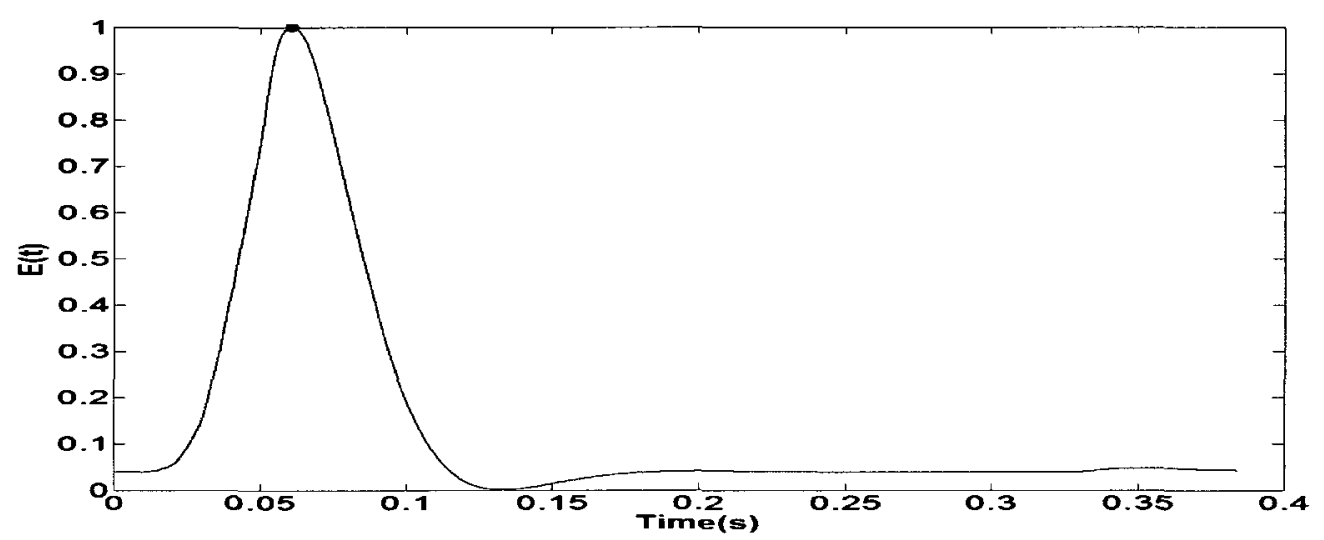

Figure 5.26: Normalized energy envelope and peaks of low-pass filtered dry cough at $100 \mathrm{~Hz}$.

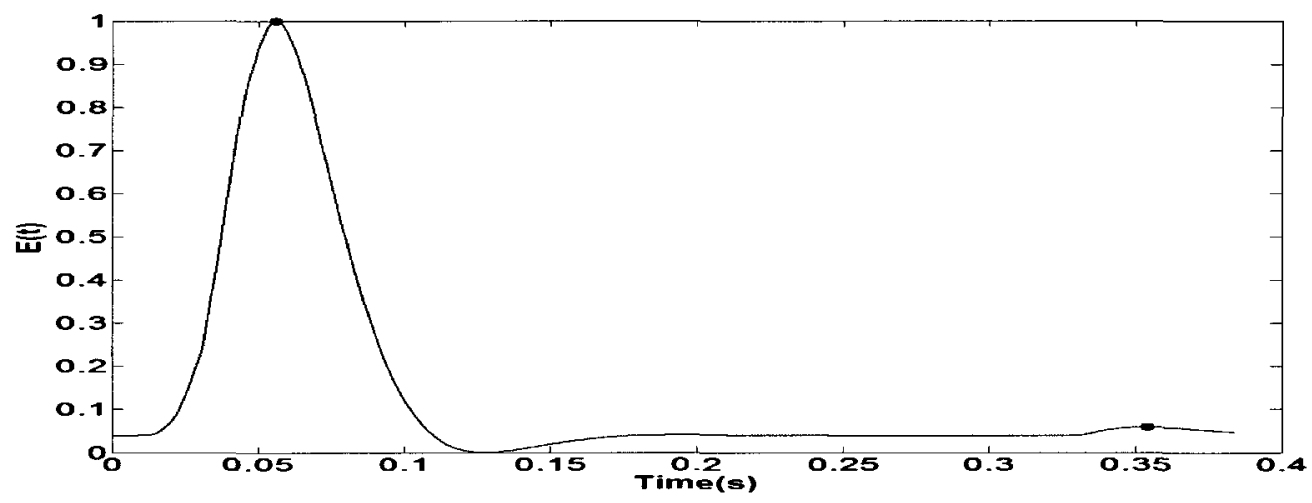

Figure 5.27: Normalized energy envelope and peaks of low-pass filtered dry cough at $300 \mathrm{~Hz}$. 


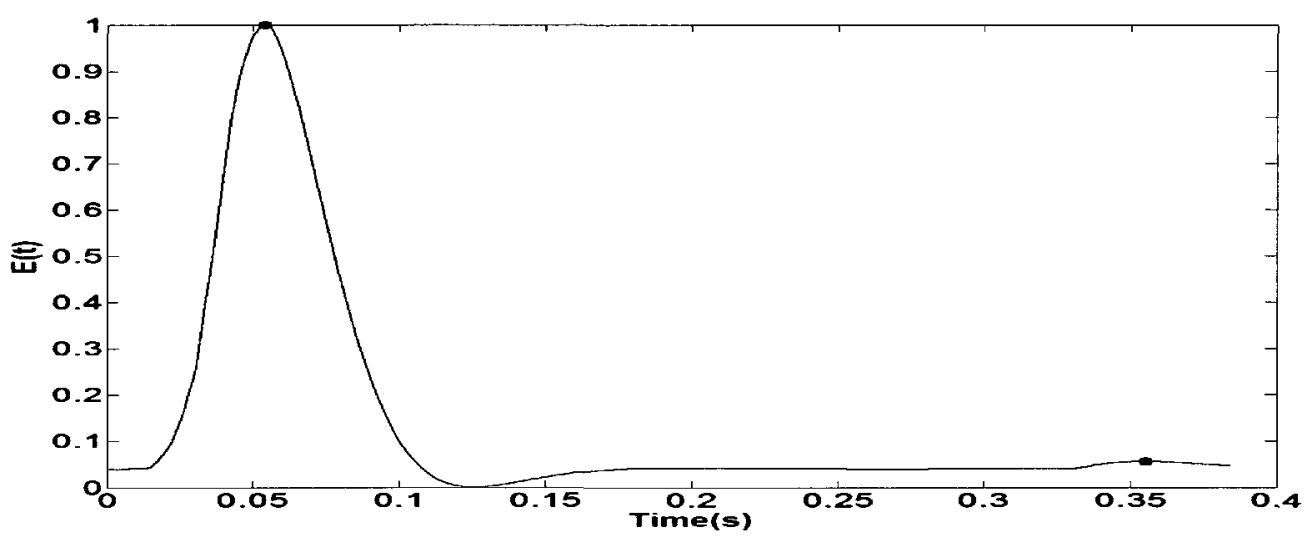

Figure 5.28: Normalized energy envelope and peaks of low-pass filtered dry cough at $500 \mathrm{~Hz}$.

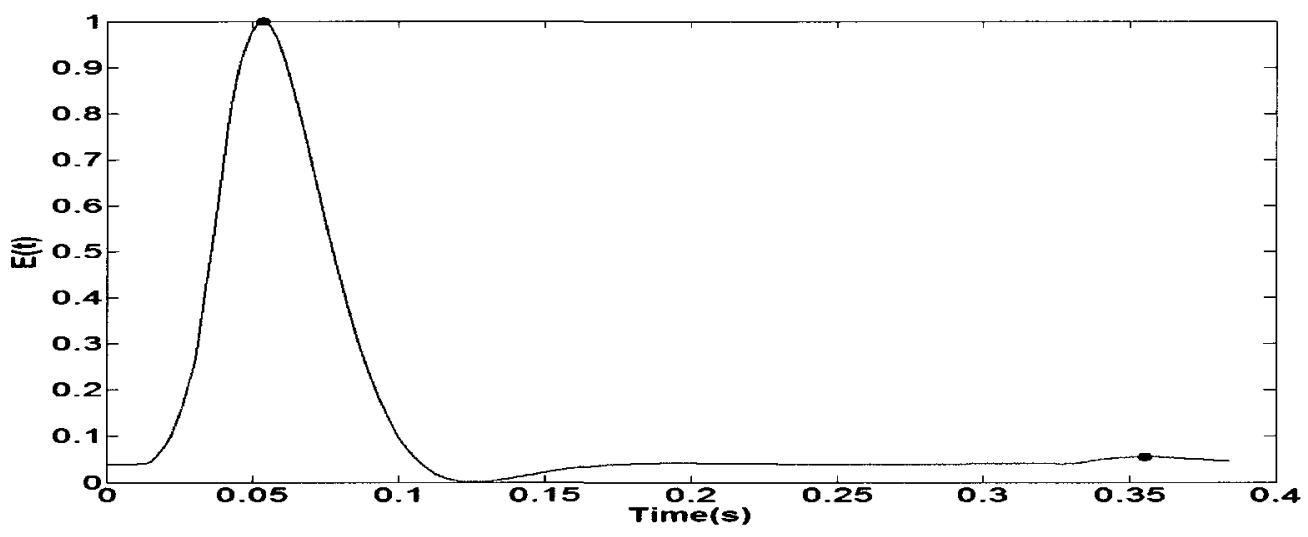

Figure 5.29: Normalized energy envelope and peaks of low-pass filtered dry cough at $700 \mathrm{~Hz}$. 


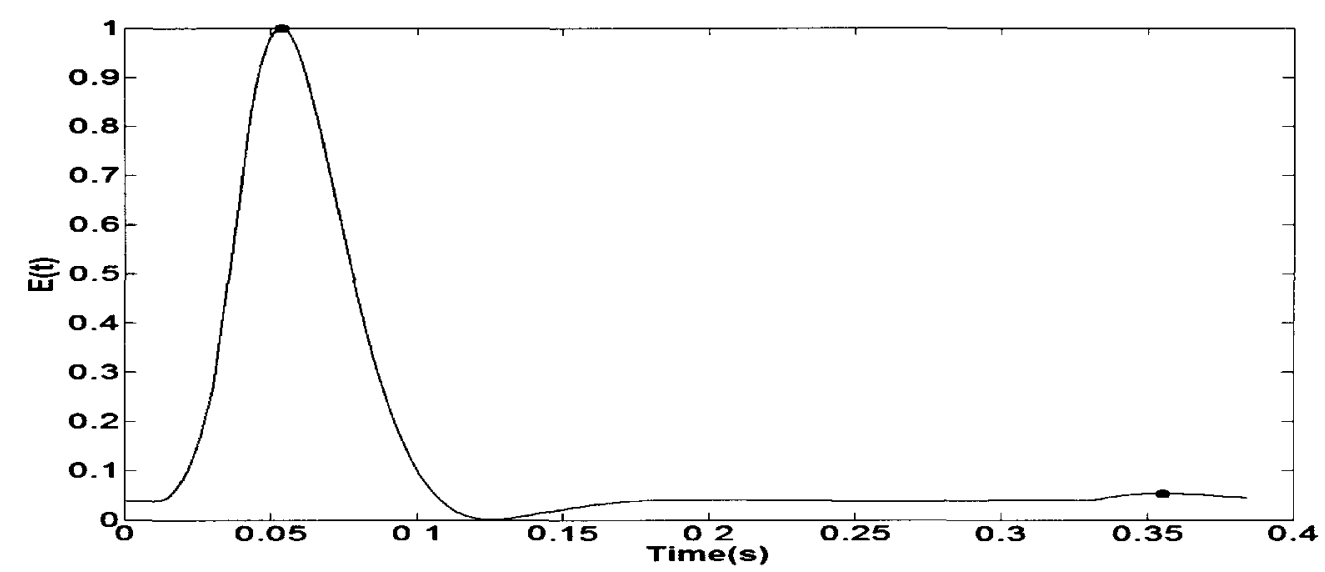

Figure 5.30: Normalized energy envelope and peaks of low-pass filtered dry cough at $900 \mathrm{~Hz}$.

\subsubsection{Wet Cough Signal}

Similar to Section 5.4.1, this section shows the steps involved in extracting Feature 1 using a low-pass filter (LPF) for wet cough signals. Figure 5.31 shows a wet cough sample. Figure 5.32, Figure 5.33, Figure 5.34, Figure 5.35, and Figure 5.36 show the lowpass filtered signal of the wet cough sample with different cut-off frequencies. 


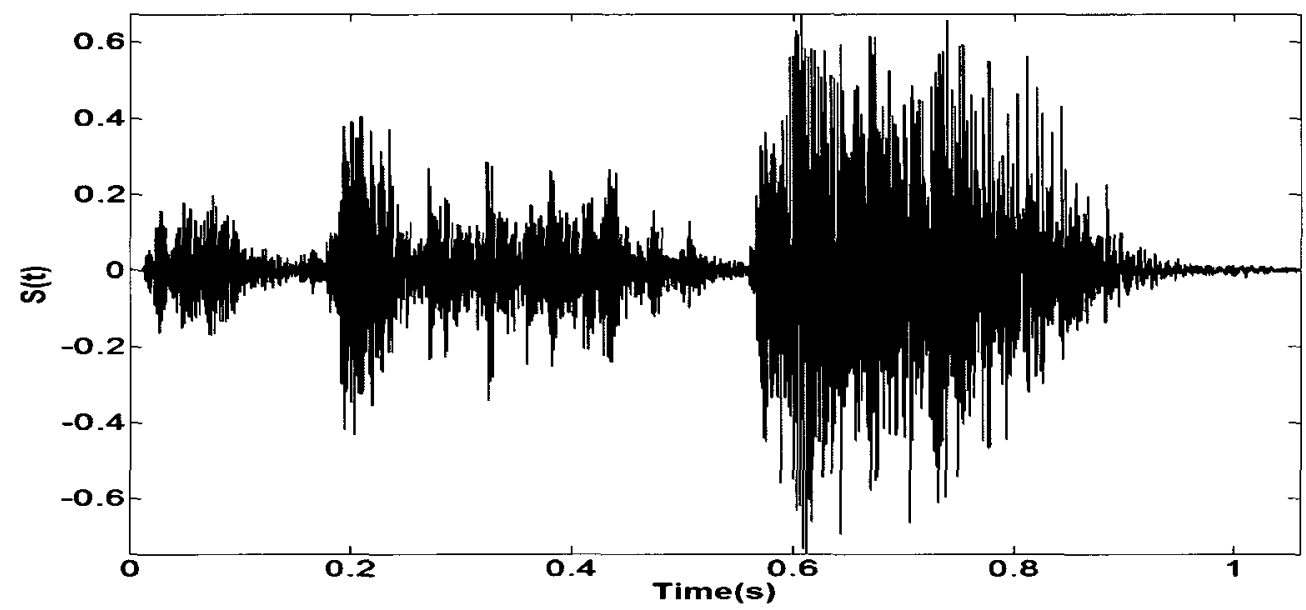

Figure 5.31: Wet cough sample.

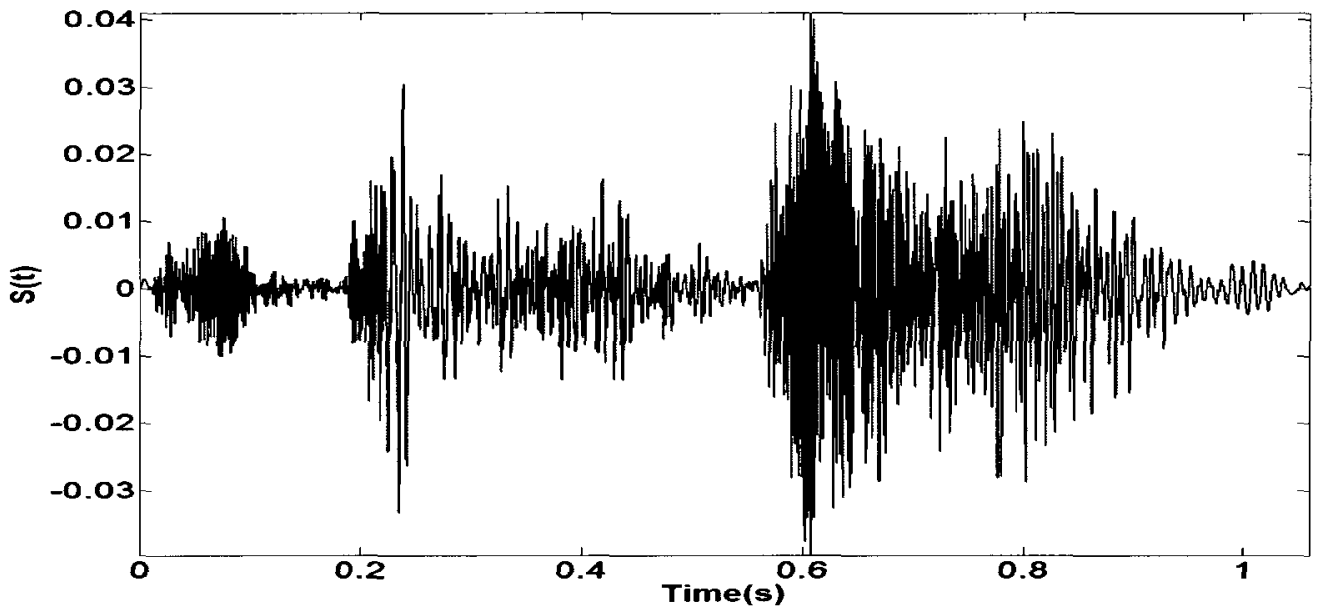

Figure 5.32: Low-pass filtered wet cough sample at $100 \mathrm{~Hz}$. 


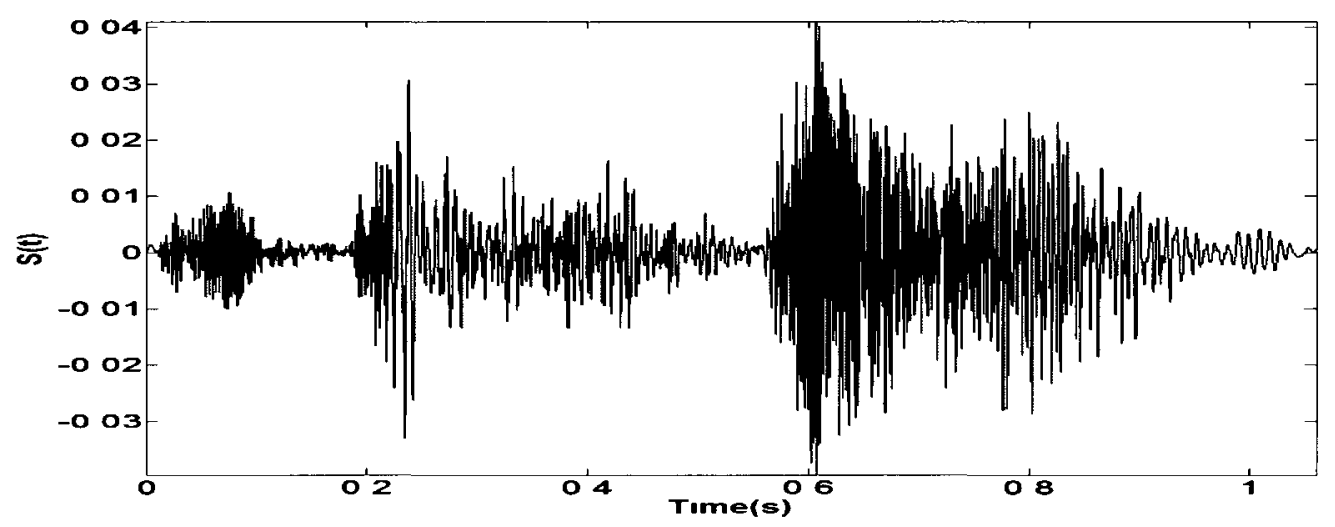

Figure 5.33: Low-pass filtered wet cough sample at $300 \mathrm{~Hz}$.

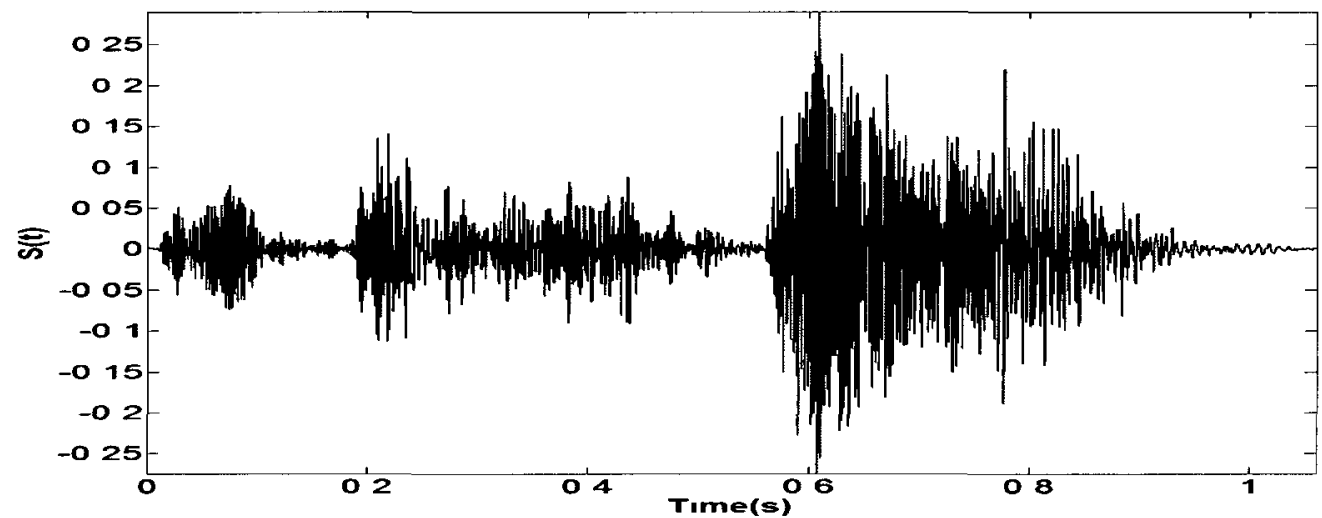

Figure 5.34: Low-pass filtered wet cough sample at $500 \mathrm{~Hz}$.

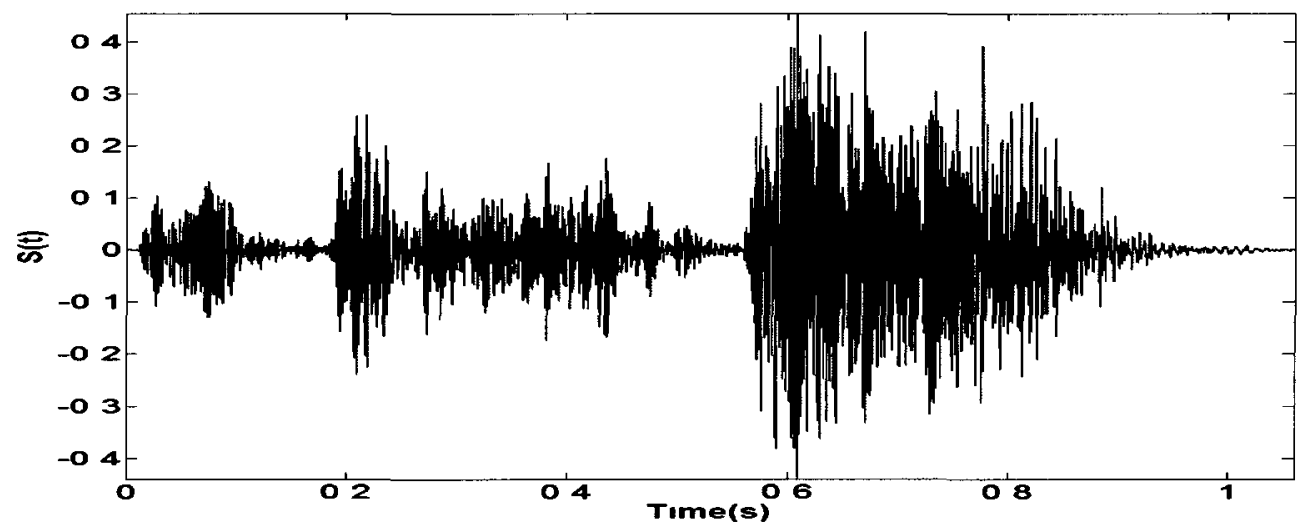

Figure 5.35: Low-pass filtered wet cough sample at $700 \mathrm{~Hz}$. 


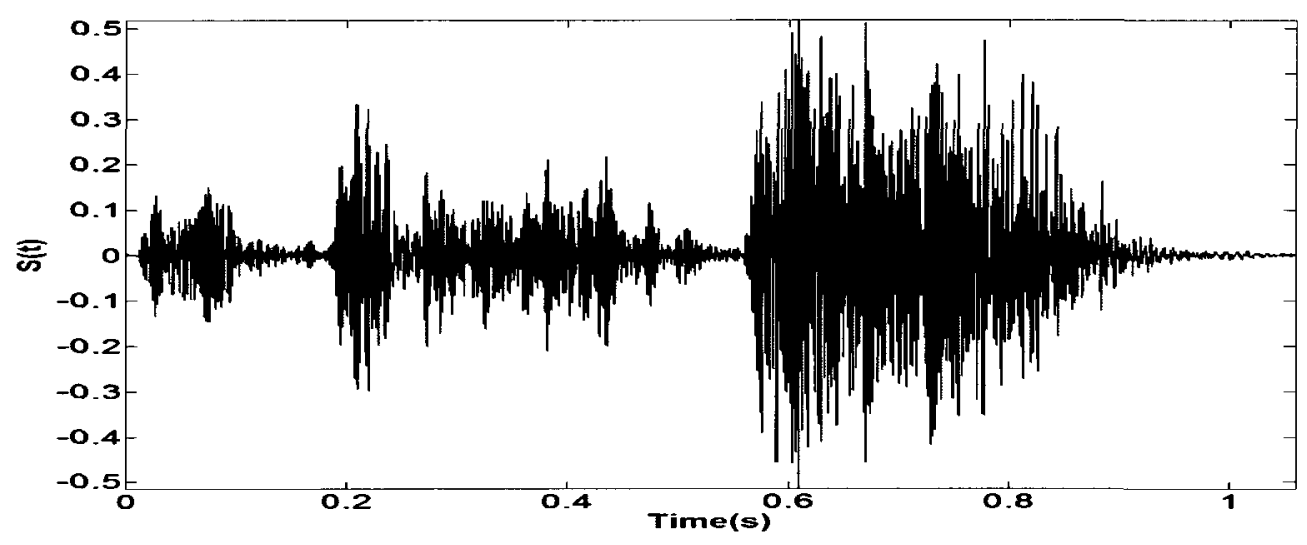

Figure 5.36: Low-pass filtered wet cough sample at $900 \mathrm{~Hz}$.

The following figures, Figure 5.37, Figure 5.38, Figure 5.39, Figure 5.40, and Figure 5.41 show the signal squared, $\mathrm{S}^{2}(\mathrm{t})$, of the low-pass filtered wet cough sample.

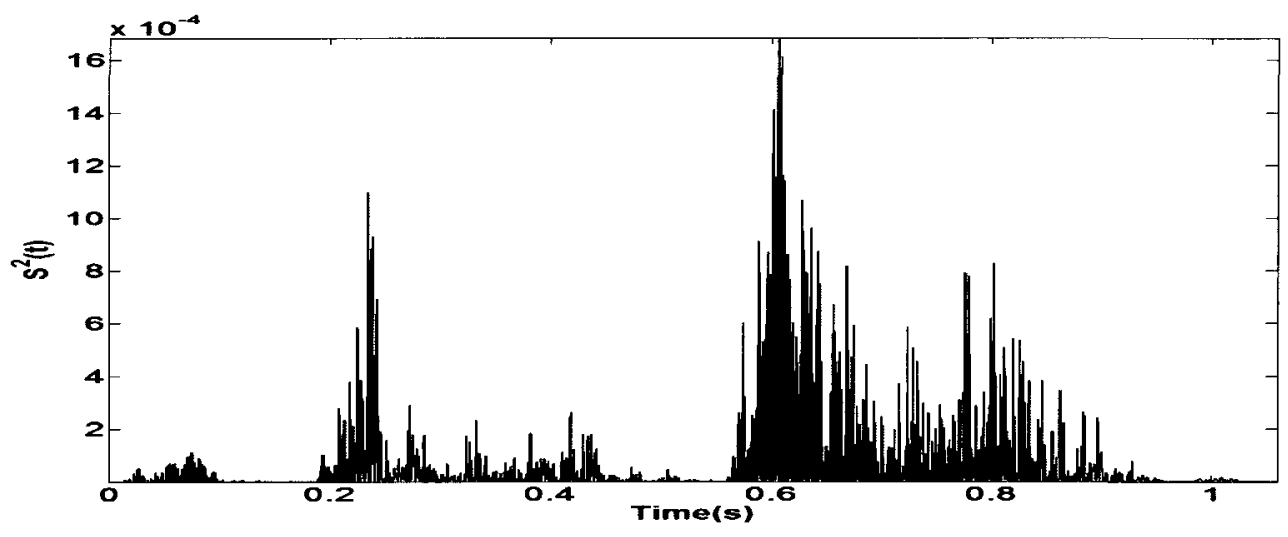

Figure 5.37: Signal squared of a low-pass filtered wet cough sound at $100 \mathrm{~Hz}$. 


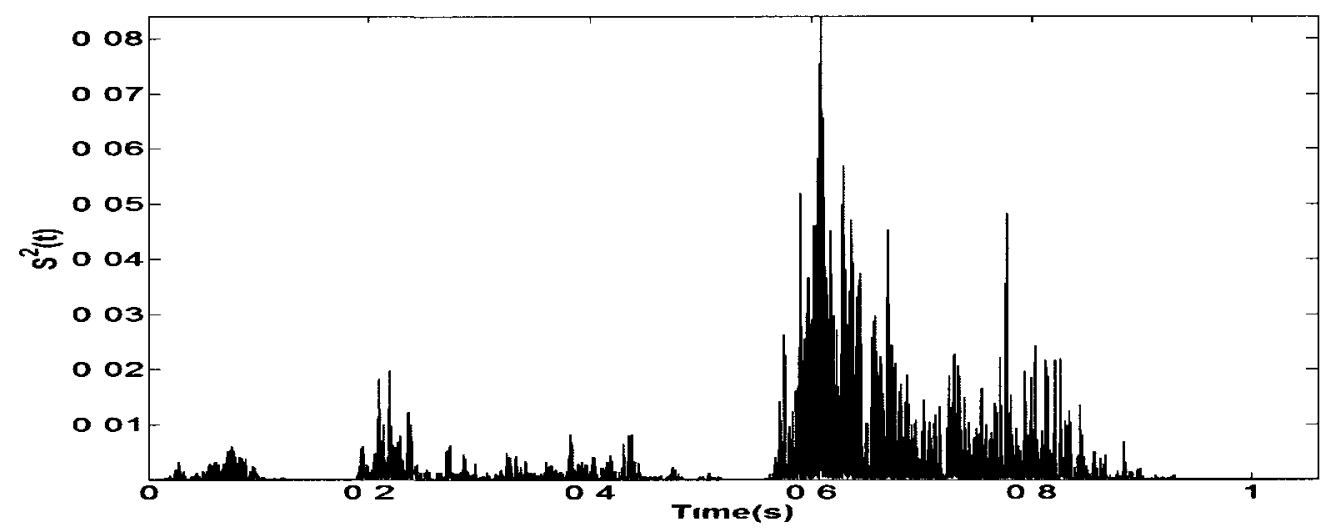

Figure 5.38: Signal squared of a low-pass filtered wet cough sound at $300 \mathrm{~Hz}$.

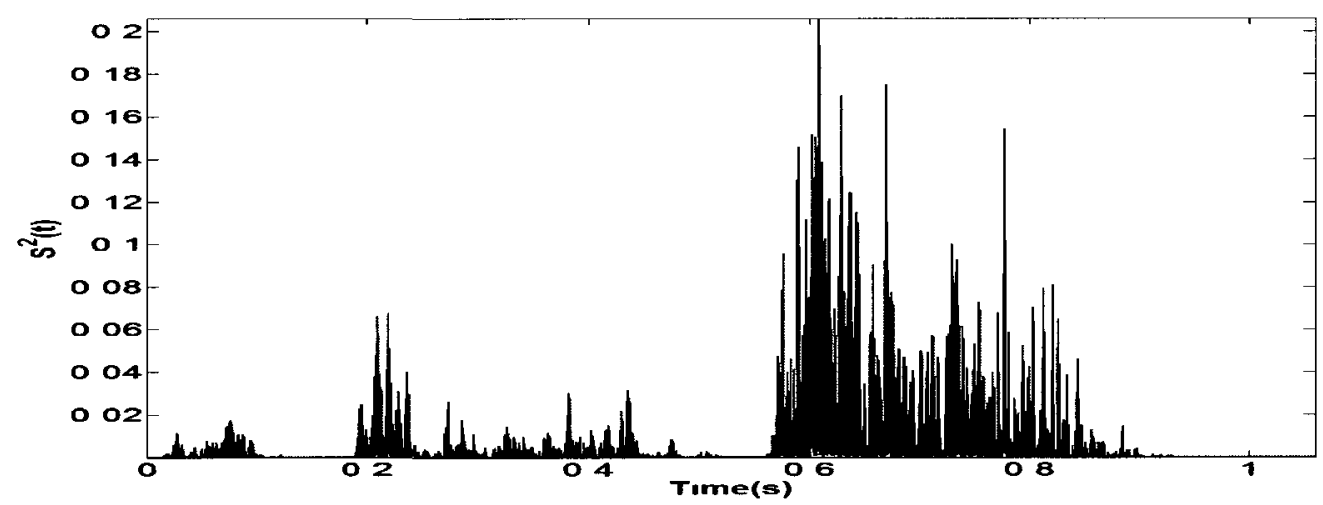

Figure 5.39: Signal squared of a low-pass filtered wet cough sound at $500 \mathrm{~Hz}$.

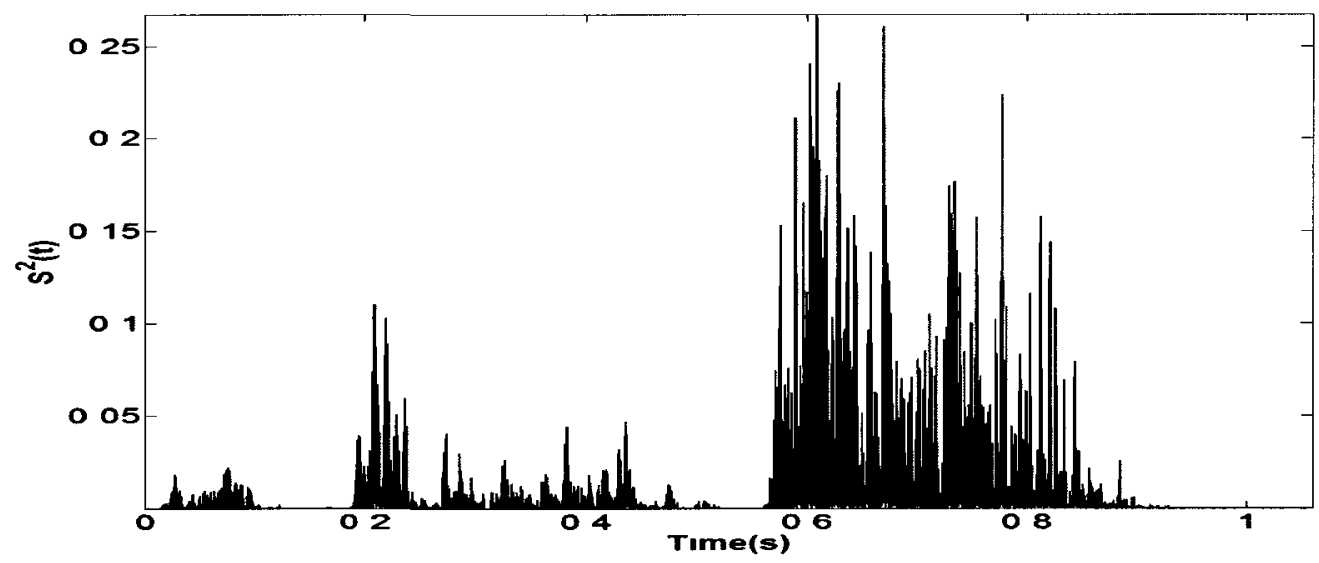

Figure 5.40: Signal squared of a low-pass filtered wet cough sound at $700 \mathrm{~Hz}$. 


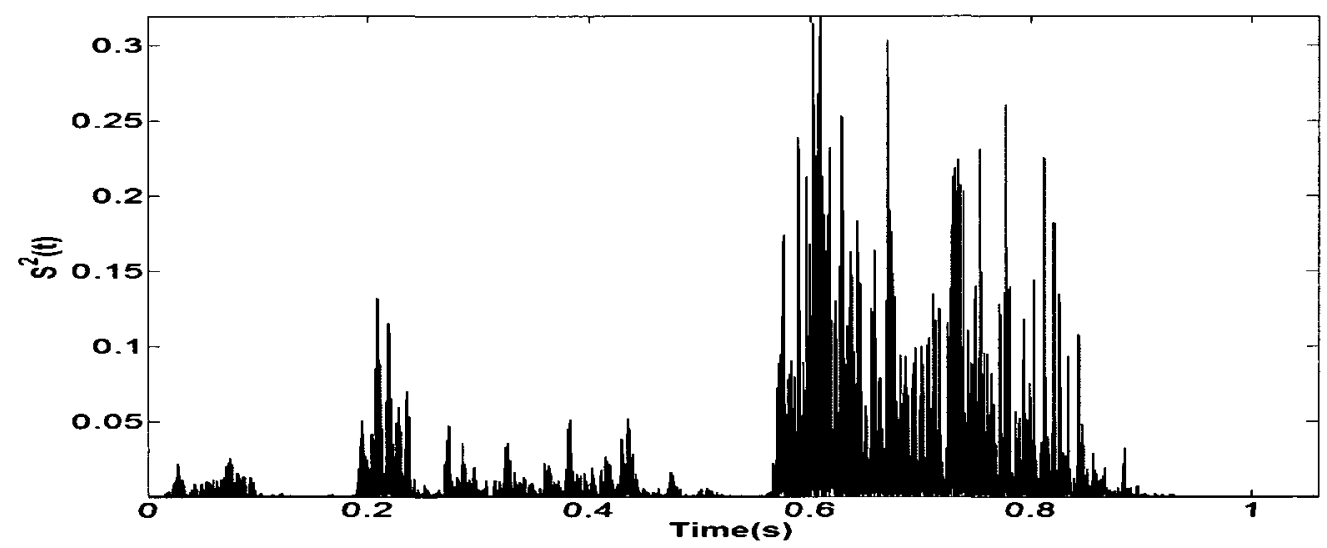

Figure 5.41: Signal squared of a low-pass filtered wet cough sound at $900 \mathrm{~Hz}$.

Energy envelopes and peaks of the low-pass filtered wet cough signal at different frequency bands are computed and depicted in Figure 5.42, Figure 5.43, Figure 5.44, Figure 5.45, and Figure 5.46

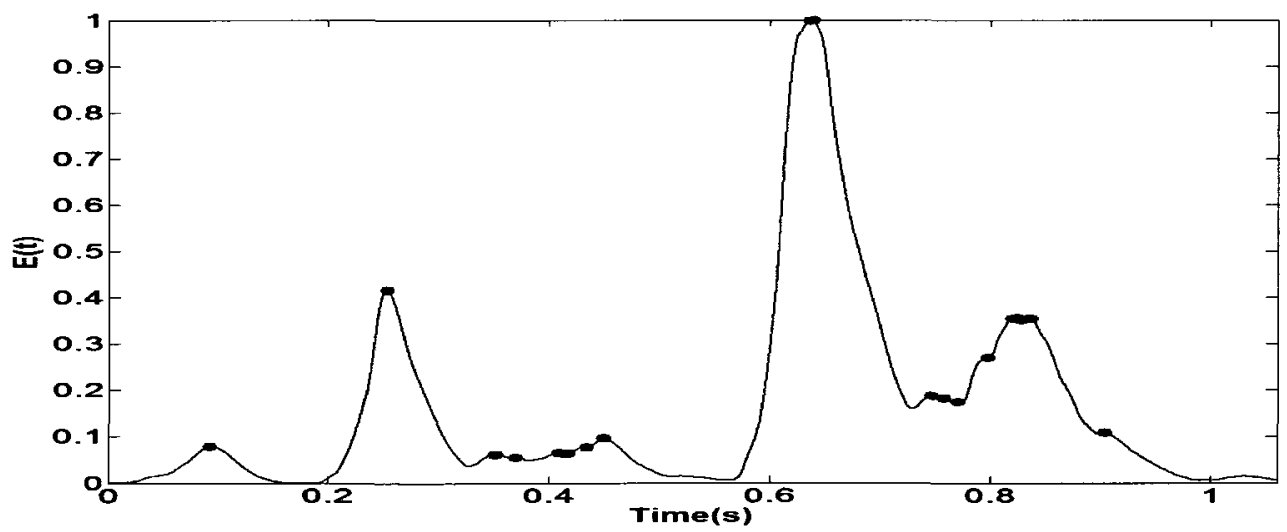

Figure 5.42: Normalized energy envelope and peaks of low-pass filtered wet cough at $100 \mathrm{~Hz}$. 


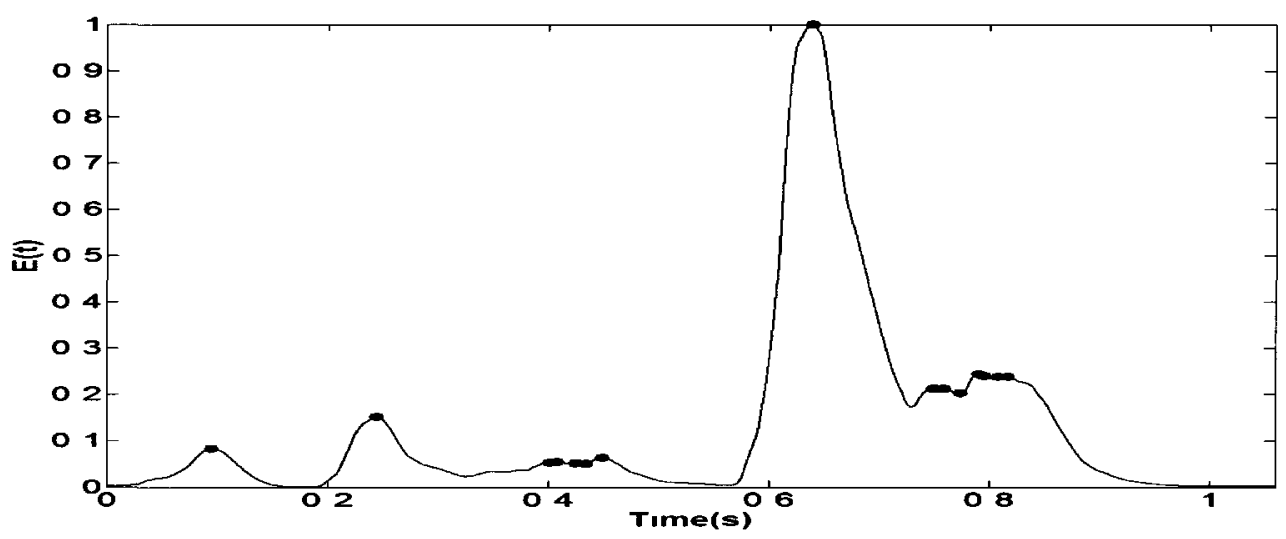

Figure 5.43: Normalized energy envelope and peaks of low-pass filtered wet cough at $300 \mathrm{~Hz}$.

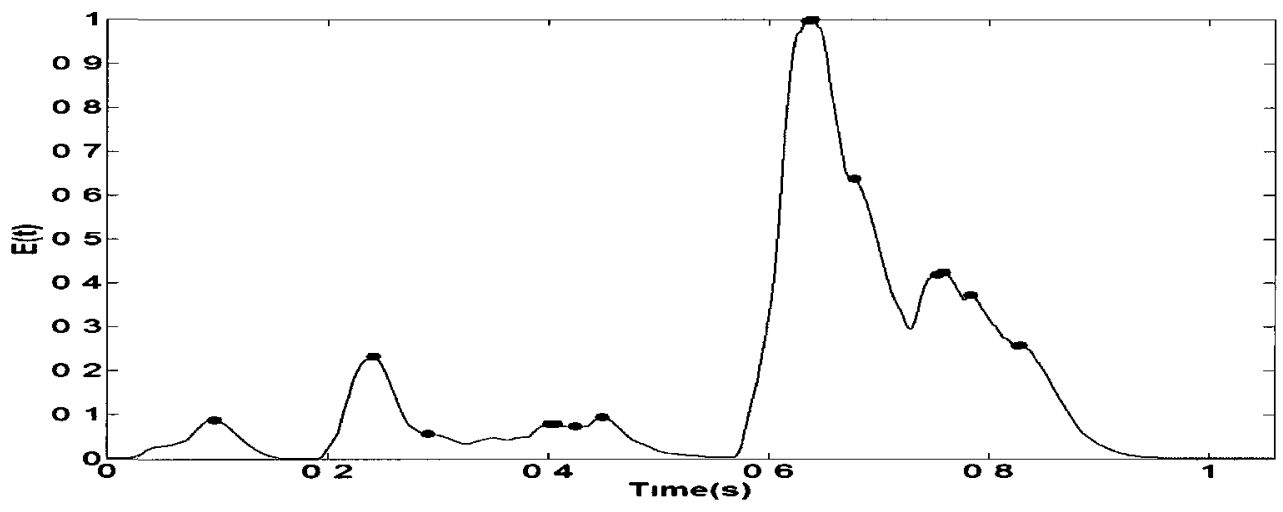

Figure 5.44: Normalized energy envelope and peaks of low-pass filtered wet cough at 500Hz. 


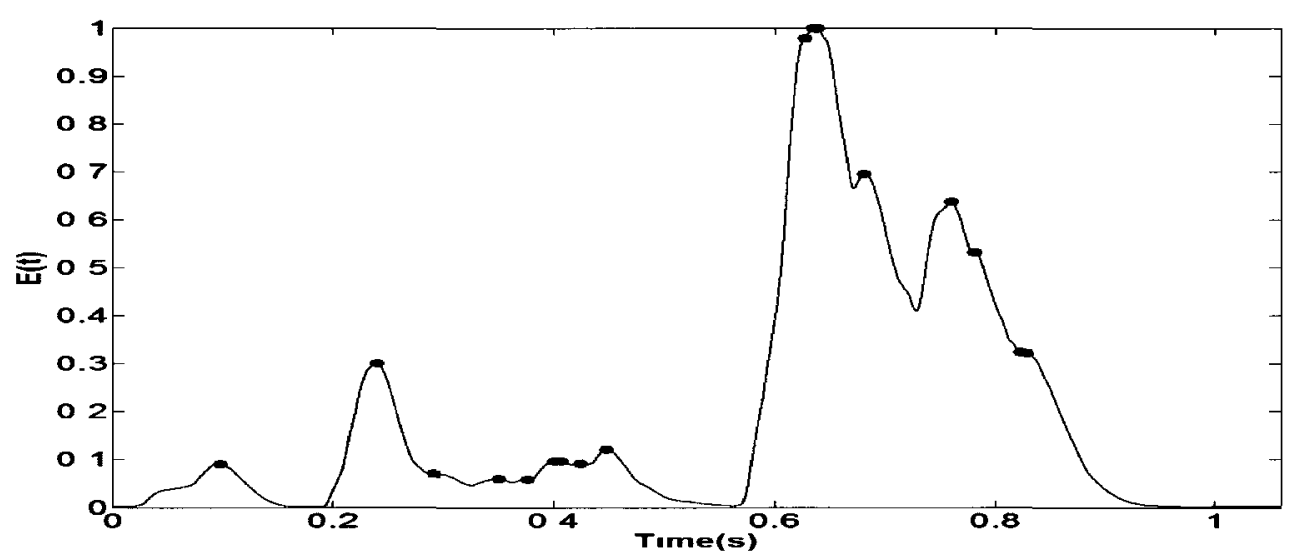

Figure 5.45: Normalized energy envelope and peaks of low-pass filtered wet cough at $\mathbf{7 0 0 H z}$.

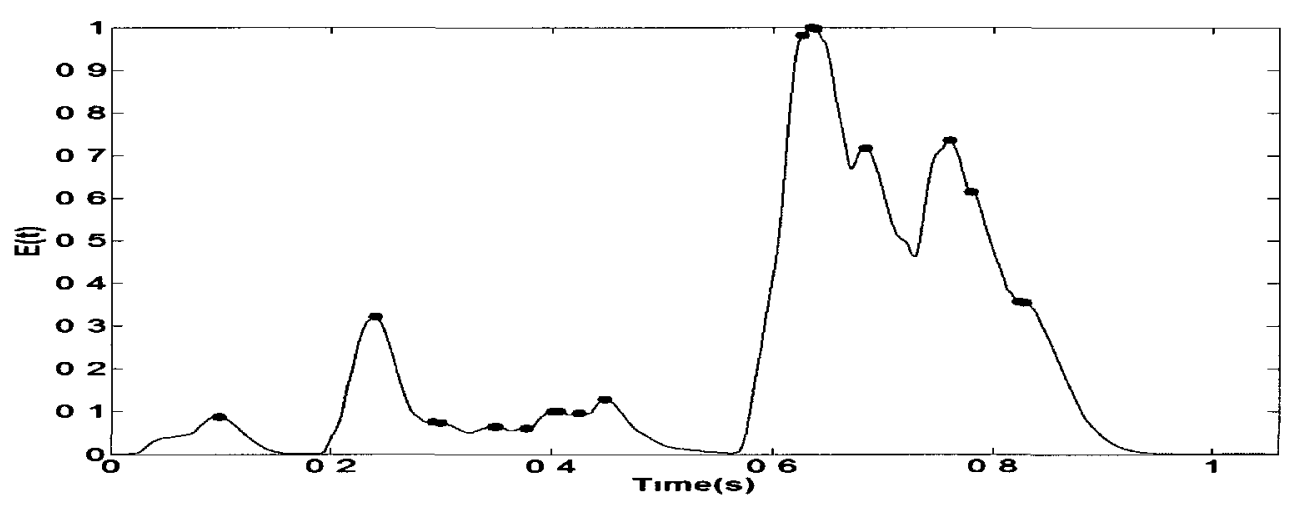

Figure 5.46: Normalized energy envelope and peaks of low-pass filtered wet cough at $900 \mathrm{~Hz}$.

Clearly, there is still a significant difference in energy envelope of both dry and wet cough samples. Similar to Section 5.3.5, the energy envelope of the wet cough sample tends to have a random shape, whereas a consistent shape is being observed for the dry cough energy envelope. In order to further verify the results of this section, the same set 
of experiments were performed on more cough samples and will be explained further in Section 5.7.

\subsection{Frequency Domain Feature}

As mentioned in previous section, wet cough signals show more power at lower frequency regions than dry cough signals. Based on this characteristic, the following algorithm was implemented to extract a feature in the frequency domain. The development of this feature will be explained in detail in the following sections.

\subsection{Power Ratio Estimation}

This section introduces an algorithm which extracts the power ratio of two frequency bands of the second phase of the cough signal. This will be referred to as Feature 2 . The algorithm is depicted in Figure 5.47.

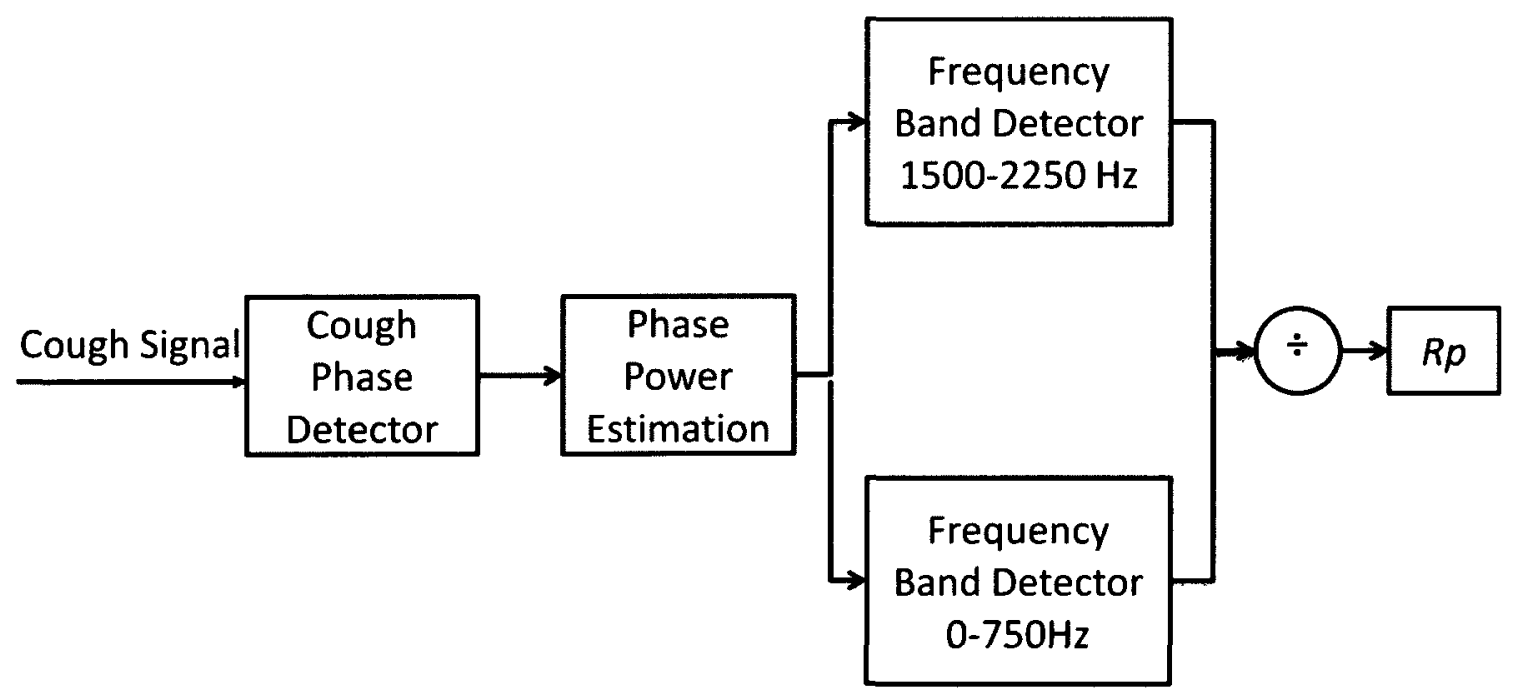

Figure 5.47: Power ratio estimation feature extraction algorithm. 


\subsubsection{Cough Phase Detector}

In this section, each cough recording was divided into 3 phases, using two algorithms. Each algorithm will be discussed in the following sections.

A. The first algorithm divides each cough sound into 4 equal parts as shown in Figure 5.48. The first part represents Phase 1, the second and third parts represent Phase 2 and the last part represents Phase 3 . The 3 phases of each cough sound were detected using this algorithm. This algorithm has its own advantages and disadvantages. Its advantage is that the 3 phases of each cough sound would be detected automatically; however, since the length of the cough sounds are different, detecting each phase might not be as accurate as desired.

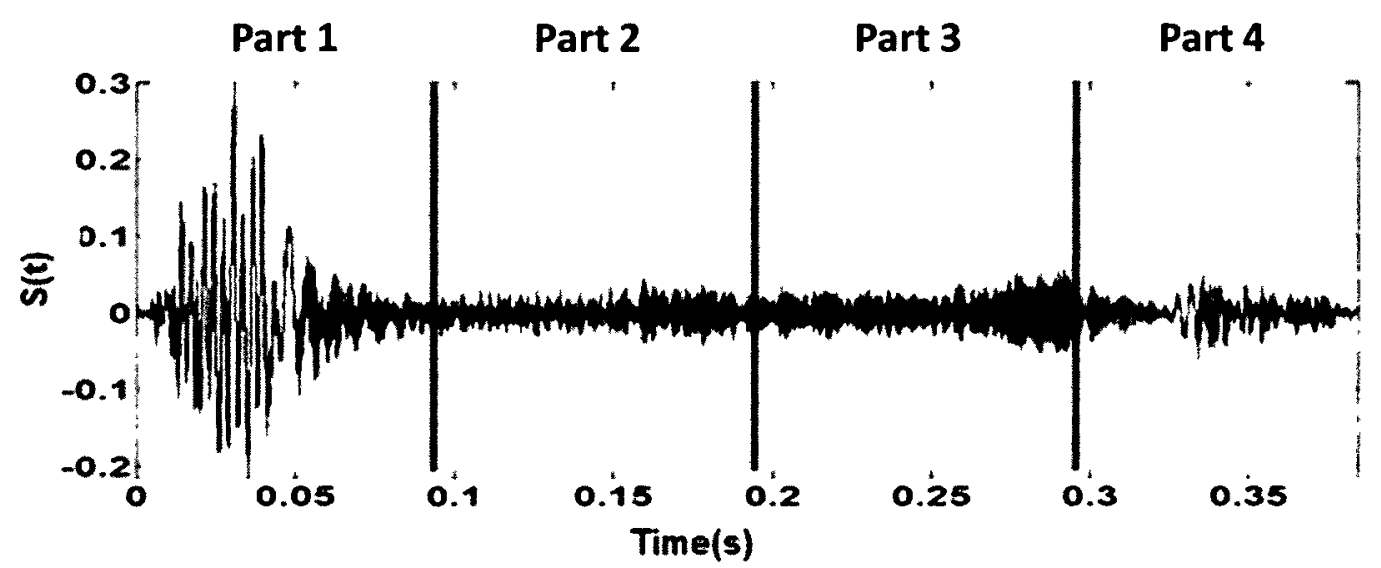

Figure 5.48: Cough sound divided into 4 equal parts. 
B. The second algorithm for phase detection was to use the start and end point of each phase. The beginning of the cough sound was used as the start point of Phase 1. The start of the second phase was chosen when the sound amplitude had reduced significantly from its initial peak [61]. The start of Phase 3 was selected when there was a rise in the sound amplitude after the second phase.

The second algorithm was used in this thesis, since detecting the 3 phases was more accurate.Figure 5.49 and Figure 5.50 show a dry cough signal and a wet cough signal with their 3 phases respectively. 

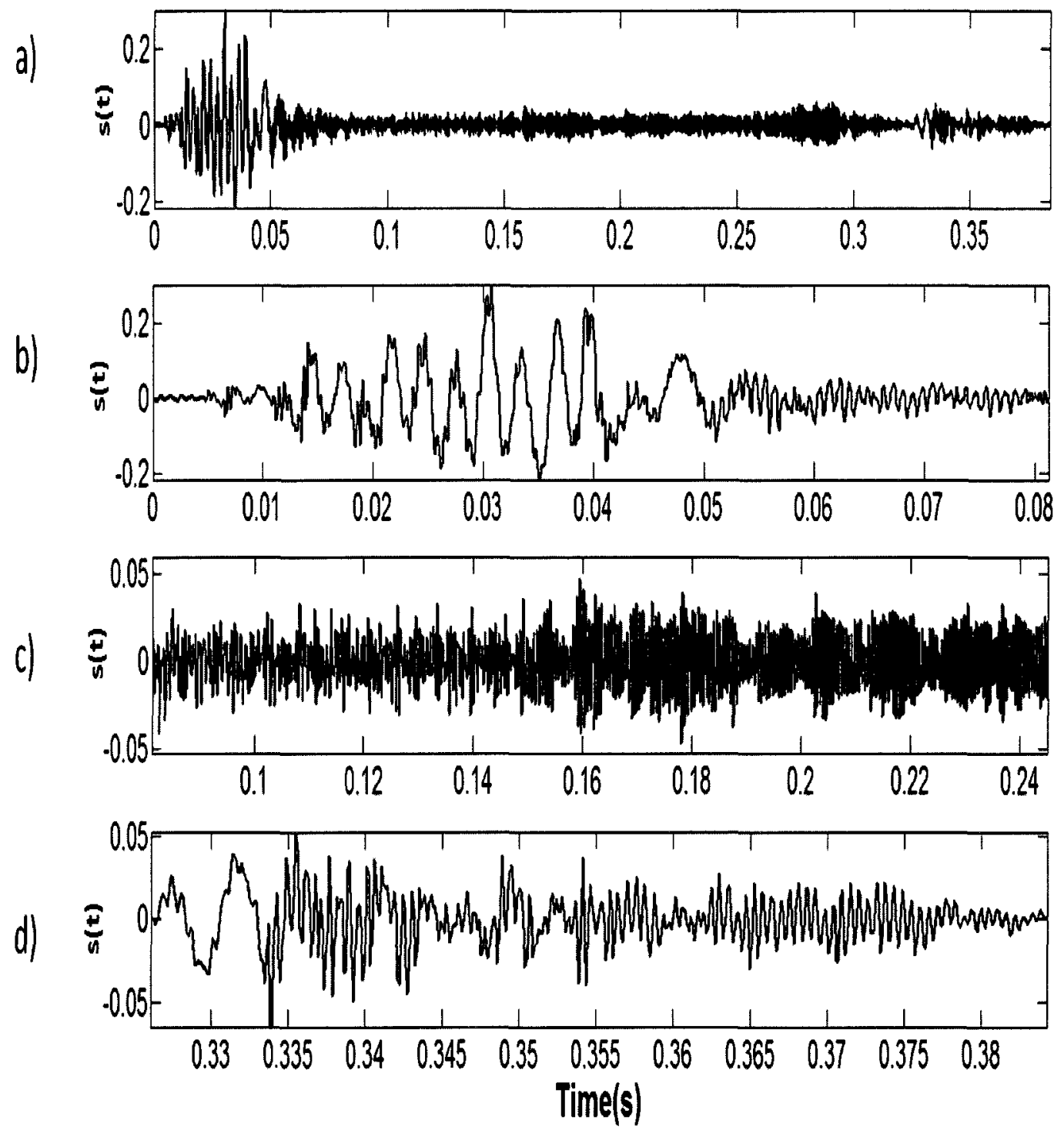

Figure 5.49: a) Dry cough signal, b) Phase 1, c) Phase 2, and d) Phase 3. 
a)

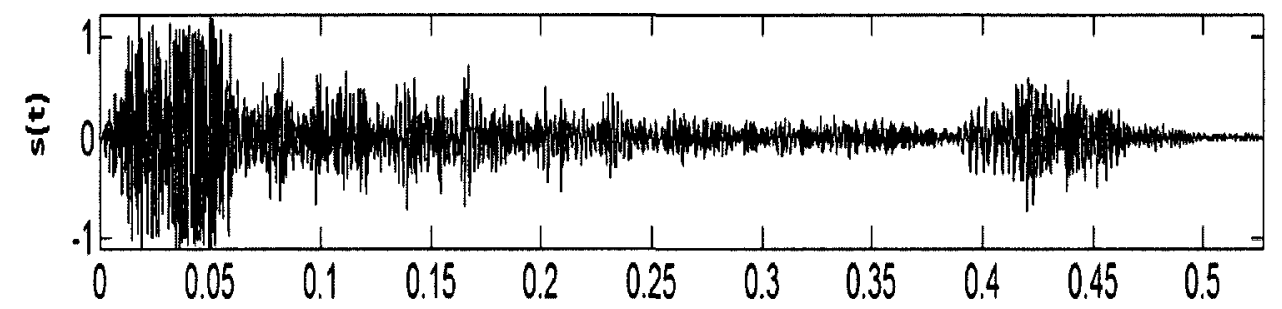

b)
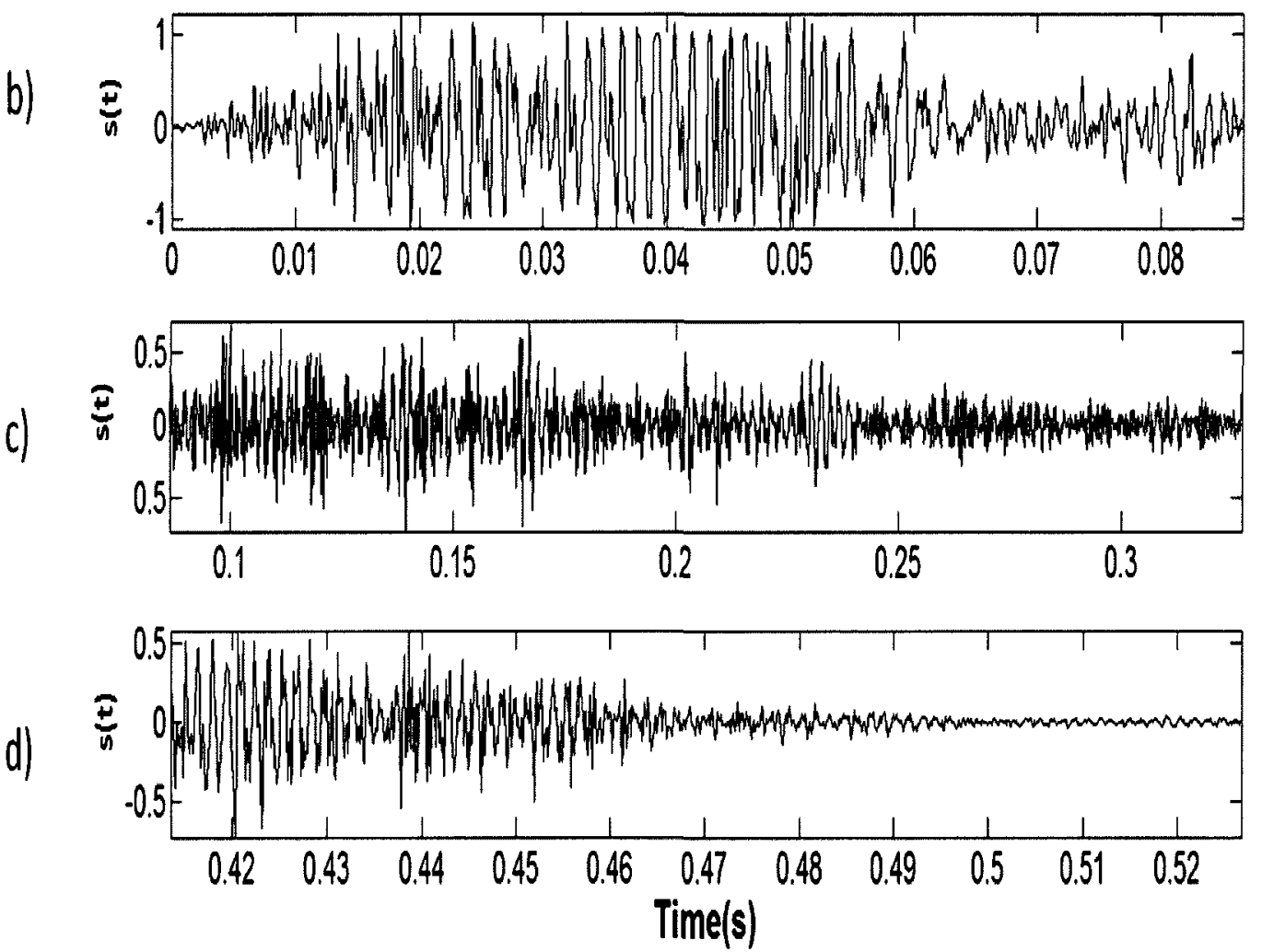

Figure 5.50: a) Wet cough signal, b) Phase 1, c) Phase 2, and d) Phase 3. 


\subsubsection{Phase Power Estimation}

The power of each phase was calculated in this step. P1, P2 and P3 are the power of Phase 1, Phase 2 and Phase 3 respectively. P1, P2 and P3 for a dry and a wet cough are depicted in Figure 5.51 and Figure 5.52.

a)

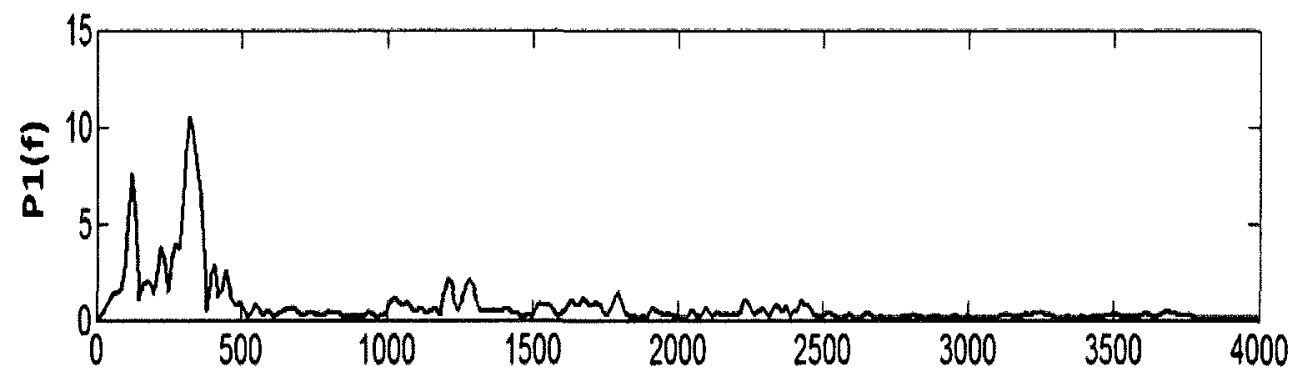

b)

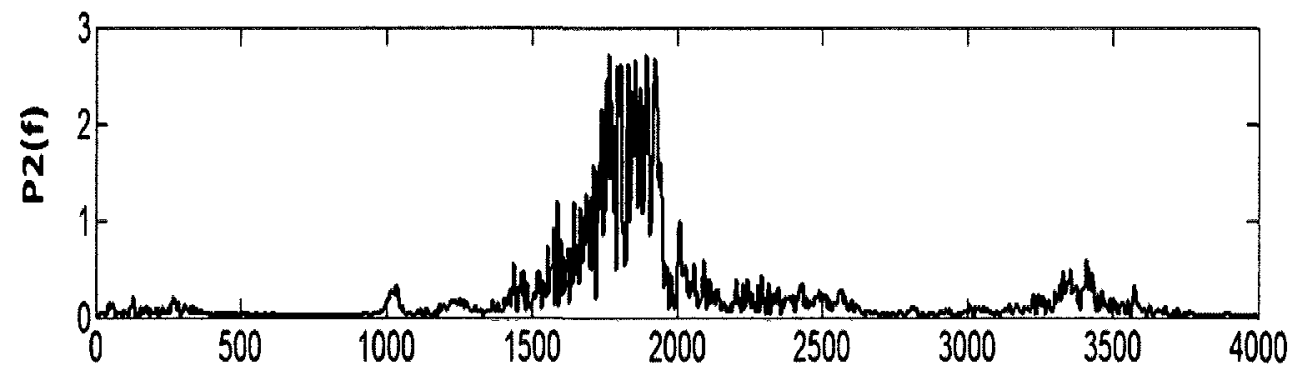

c)

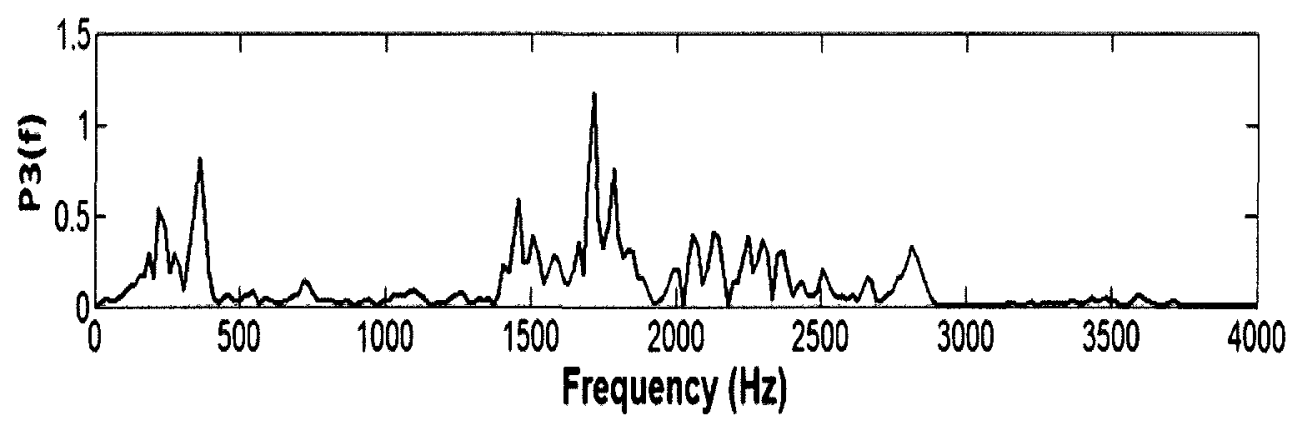

Figure 5.51: Power of a dry cough a) Phase 1, b) Phase 2, and c) Phase 3. 
a)

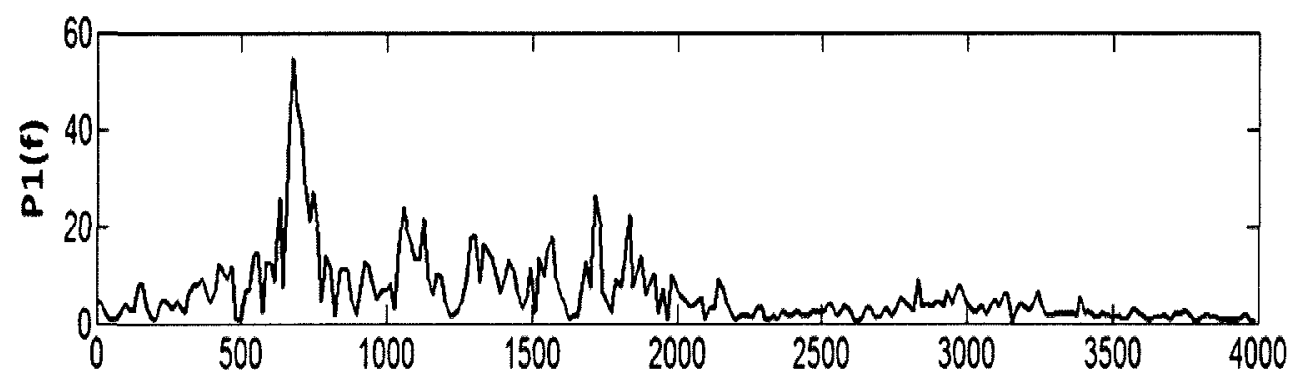

b)

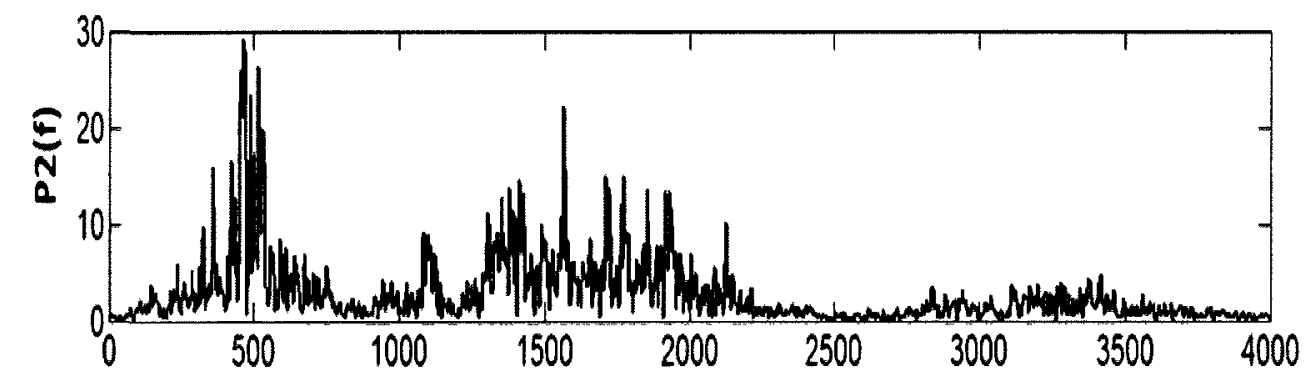

c)

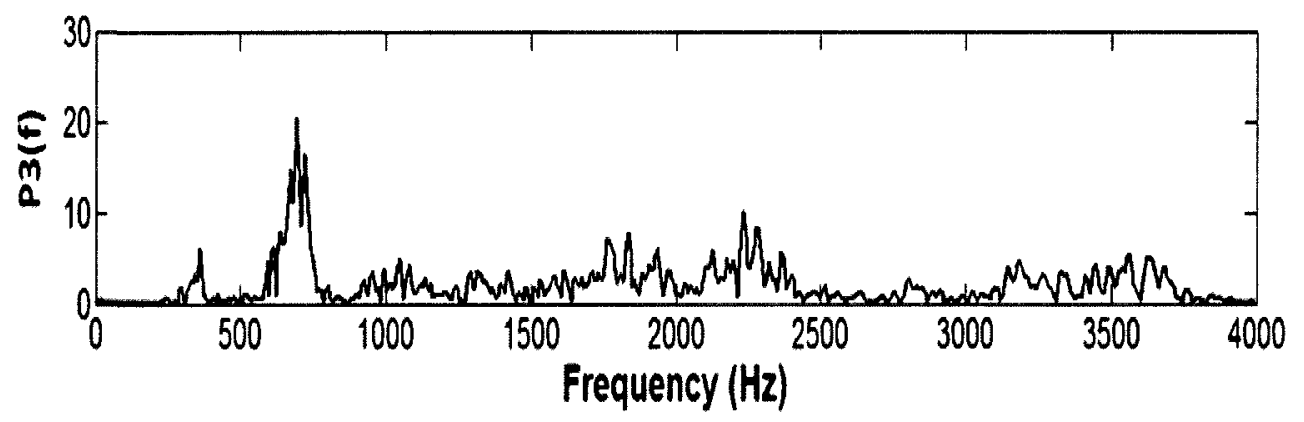

Figure 5.52: Power of a wet cough a) Phase 1, b) Phase 2, and c) Phase 3.

Comparing the power of each phase of both dry and wet cough shows that Phase 1 and Phase 3 of both dry and wet cough signals are similar; however, some differences can be seen from Phase 2 of each type of cough. Therefore in this feature algorithm Phase 2 will be further examined. 
As mentioned previously, the recording method of each cough recording was different and since the power of a signal is dependent on the distance of the source to the microphone, $\mathrm{P} 2$ was normalized by dividing it by the total power of Phase 1, P1 as shown in (1).

$$
P 2_{\text {norm }}(f)=\frac{P 2(f)}{\sum_{f=0}^{f=4 k} P 1(f)}
$$

where P1(f) and P2(f) are the power of the Phase 1 and Phase 2 at frequency $f$ respectively. $\mathrm{P} 2_{\text {norm }}(\mathrm{f})$ is the normalized power of Phase 2.

For dry cough signals, a large peak was observed around $1500-2500 \mathrm{~Hz}$ as depicted in Figure 5.53a, Figure 5.53b and Figure 5.53c. On the other hand, for wet cough signals, the peak was observed around $0-750 \mathrm{~Hz}$ as depicted in Figure 5.53d, Figure 5.53e and Figure 5.53f. 

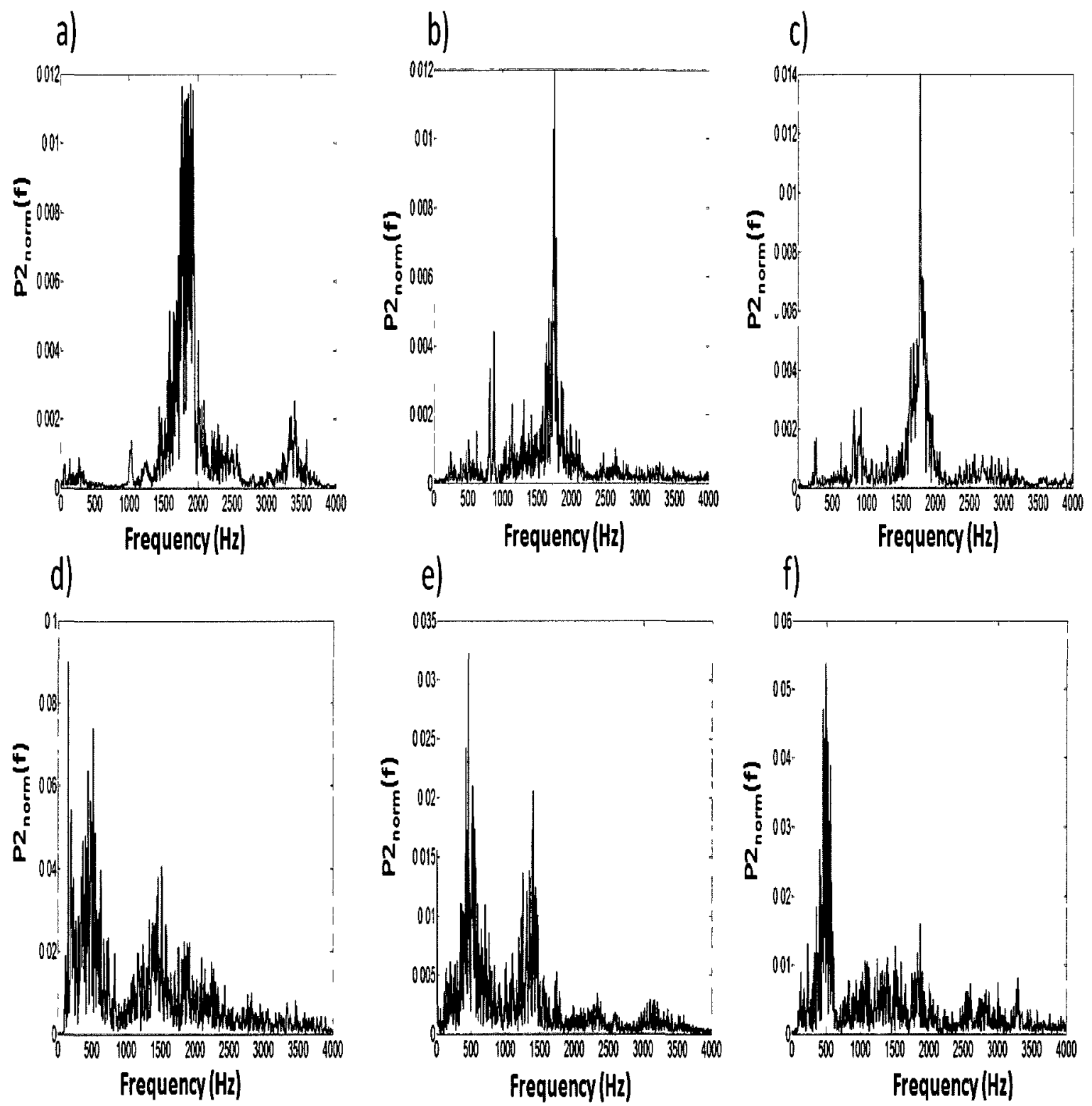

Figure 5.53: $P 2_{\text {norm}}(f)$ of a) Dry sample 1, b) Dry sample 2, c) Dry sample 3, d) Wet sample 1, e) Wet sample2, and d) Wet sample 3. 


\subsubsection{Power Ratio Estimation}

From the previous section, it was observed that there is a peak at $1500-2250 \mathrm{~Hz}$ for $\mathrm{P} 2_{\text {norm }}(\mathrm{f})$ of dry cough sounds. On the other hand, at $0-750 \mathrm{~Hz}$, there is a peak for $\mathrm{P} 2_{\text {norm }}(\mathrm{f})$ of wet cough sounds. Based on this observation, the power ratio, $\mathrm{Rp}$, was calculated as in (2).

$$
R p=\frac{\sum_{f=1500}^{f=2250} P 2_{\text {norm }}(f)}{\sum_{f=0}^{f=750} P 2_{\text {norm }}(f)}
$$

where $P 2_{\text {norm }}(f)$ is the normalized power of Phase 2 .

\subsection{Results}

\subsubsection{Results of Feature 1 Extraction Algorithm using Band-Pass Filter (BPF)}

The Feature 1 extraction algorithm was run for both dry and wet cough samples for different frequency bands. The number of peaks for each frequency band of each cough was recorded and is reported in Table 6 , Table 7 , Table 8 , and Table 9. 
Table 6: Number of peaks of dry cough samples for frequency bands between $0-500 \mathrm{~Hz}$

\begin{tabular}{|c|c|c|c|c|c|c|c|c|c|c|}
\hline 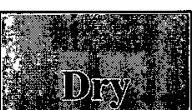 & 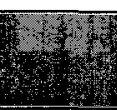 & 40 & S18 & 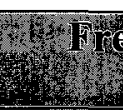 & onency & Bango & $(1-7)$ & (5) & 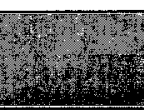 & S \\
\hline (Conglo & $\begin{array}{r}0-50 \\
(1) \\
0\end{array}$ & $\begin{array}{l}50= \\
50 \\
100\end{array}$ & $\begin{array}{l}100= \\
150 \\
4\end{array}$ & $\begin{array}{l}1500 \\
20 \\
200 \\
2\end{array}$ & $\begin{array}{l}2000 \\
250 \\
250\end{array}$ & $\begin{array}{l}2500 \\
300 \\
\end{array}$ & $\begin{array}{c}300 \\
650 \\
6\end{array}$ & $\begin{array}{c}3 a 00 \\
400 \\
40\end{array}$ & $\begin{array}{l}4000 \\
45(0) \\
\end{array}$ & $\begin{array}{l}4500 \\
500 \\
5\end{array}$ \\
\hline Sanople1 & 1 & 1 & 1 & 2 & 2 & 2 & 1 & 2 & 1 & 1 \\
\hline Sampole2 & 1 & 1 & 1 & 1 & 1 & 1 & 1 & 3 & 2 & 2 \\
\hline Stamples & 1 & 1 & 2 & 1 & 1 & 1 & 1 & 3 & 3 & 3 \\
\hline Samplest & 2 & 2 & 2 & 2 & 2 & 2 & 2 & 1 & 2 & 3 \\
\hline Sampoles: & 2 & 2 & 1 & 2 & 2 & 2 & 2 & 2 & 2 & 3 \\
\hline samples & 2 & 2 & 2 & 2 & 2 & 2 & 1 & 1 & 2 & 3 \\
\hline sampile7 & 1 & 1 & 1 & 2 & 2 & 2 & 1 & 2 & 1 & 1 \\
\hline Saymple 8 & 2 & 2 & 2 & 2 & 2 & 2 & 2 & 2 & 2 & 2 \\
\hline Sampiolege & 1 & 1 & 1 & 2 & 2 & 2 & 1 & 2 & 1 & 1 \\
\hline
\end{tabular}


Table 7: Number of peaks of dry cough samples for frequency bands between $550-1000 \mathrm{~Hz}$

\begin{tabular}{|c|c|c|c|c|c|c|c|c|c|c|}
\hline ondy & 4 & 4 & Why & (1) & ginengy & Banda & 17) & (1) & (1) & Fen \\
\hline (a) & $\begin{array}{l}500 \\
550 \\
5\end{array}$ & $\begin{array}{l}550 \\
50 \\
500 \\
5\end{array}$ & $\begin{array}{r}600 \\
650 \\
6\end{array}$ & 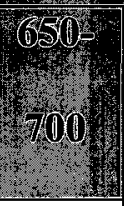 & $\begin{array}{l}700=5 \\
50 \\
5\end{array}$ & $\begin{array}{c}750= \\
8000\end{array}$ & 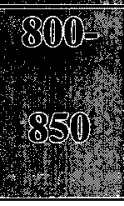 & $\begin{array}{l}850= \\
900\end{array}$ & $\begin{array}{l}200= \\
95(0)\end{array}$ & $\begin{array}{c}950= \\
1000 \\
0\end{array}$ \\
\hline Sarouplet & 2 & 1 & 1 & 1 & 2 & 2 & 1 & 1 & 1 & 2 \\
\hline Samplez & 3 & 2 & 3 & 2 & 2 & 3 & 7 & 5 & 3 & 4 \\
\hline Sample3 & 1 & 1 & 3 & 1 & 1 & 1 & 1 & 2 & 2 & 3 \\
\hline Sampilet & 2 & 3 & 2 & 2 & 1 & 2 & 4 & 3 & 2 & 2 \\
\hline Saropples & 2 & 2 & 2 & 1 & 1 & 1 & 2 & 1 & 2 & 3 \\
\hline Saropol 106 & 2 & 2 & 1 & 1 & 3 & 3 & 3 & 3 & 2 & 1 \\
\hline Sampole & 2 & 1 & 1 & 1 & 2 & 2 & 1 & 1 & 1 & 2 \\
\hline Salopple & 2 & 2 & 3 & 2 & 3 & 2 & 2 & 2 & 2 & 2 \\
\hline Samples & 2 & 1 & 1 & 1 & 2 & 2 & 1 & 1 & 1 & 2 \\
\hline
\end{tabular}


Table 8: Number of peaks of wet cough samples for frequency bands between $0-500 \mathrm{~Hz}$

\begin{tabular}{|c|c|c|c|c|c|c|c|c|c|c|}
\hline Fest & & 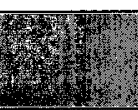 & (3) & (5) & equencr & Band & 19) & 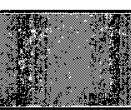 & 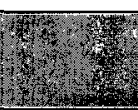 & ry \\
\hline Cough & $0=50$ & $\begin{array}{l}500 \\
100 \\
40\end{array}$ & {$\left[\begin{array}{l}0.00= \\
7 \\
50 \\
50\end{array}\right.$} & $\begin{array}{l}1500 \\
200\end{array}$ & $\begin{array}{l}2000 \\
650 \\
20\end{array}$ & $\begin{array}{l}2505 \\
3000 \\
304\end{array}$ & \begin{tabular}{|c|}
3000 \\
350 \\
330
\end{tabular} & $\begin{array}{l}3502 \\
400 \\
40\end{array}$ & $\begin{array}{l}400= \\
450 \\
4\end{array}$ & $\begin{array}{l}450= \\
500 \\
\end{array}$ \\
\hline Samplet & 22 & 18 & 13 & 14 & 4 & 6 & 3 & 4 & 11 & 12 \\
\hline sample2 & 15 & 22 & 14 & 14 & 12 & 4 & 3 & 4 & 3 & 3 \\
\hline Samples & 9 & 6 & 6 & 5 & 4 & 3 & 2 & 3 & 3 & 4 \\
\hline Sampilo4 & 12 & 14 & 6 & 10 & 9 & 6 & 9 & 11 & 4 & 6 \\
\hline Samples: & 3 & 6 & 7 & 4 & 3 & 2 & 4 & 4 & 6 & 6 \\
\hline Sample6 & 12 & 12 & 10 & 10 & 8 & 11 & 9 & 10 & 8 & 8 \\
\hline Saropolen & 1 & 2 & 2 & 2 & 9 & 7 & 6 & 5 & 4 & 5 \\
\hline Sampole & 1 & 4 & 2 & 2 & 6 & 10 & 8 & 8 & 4 & 8 \\
\hline
\end{tabular}

The number of peaks for dry cough samples was observed to be between 1 and 3; however, on the other hand, the number of peaks for wet cough signals was observed to be more than 3 for most of the frequency bands. As mentioned previously, more variation was observed in the shape of the energy envelope for wet cough signals than dry cough signals. Therefore, more peaks were detected from wet cough sounds energy envelope. 
Table 9: Number of peaks of wet cough samples for frequency bands between $550-1000 \mathrm{~Hz}$

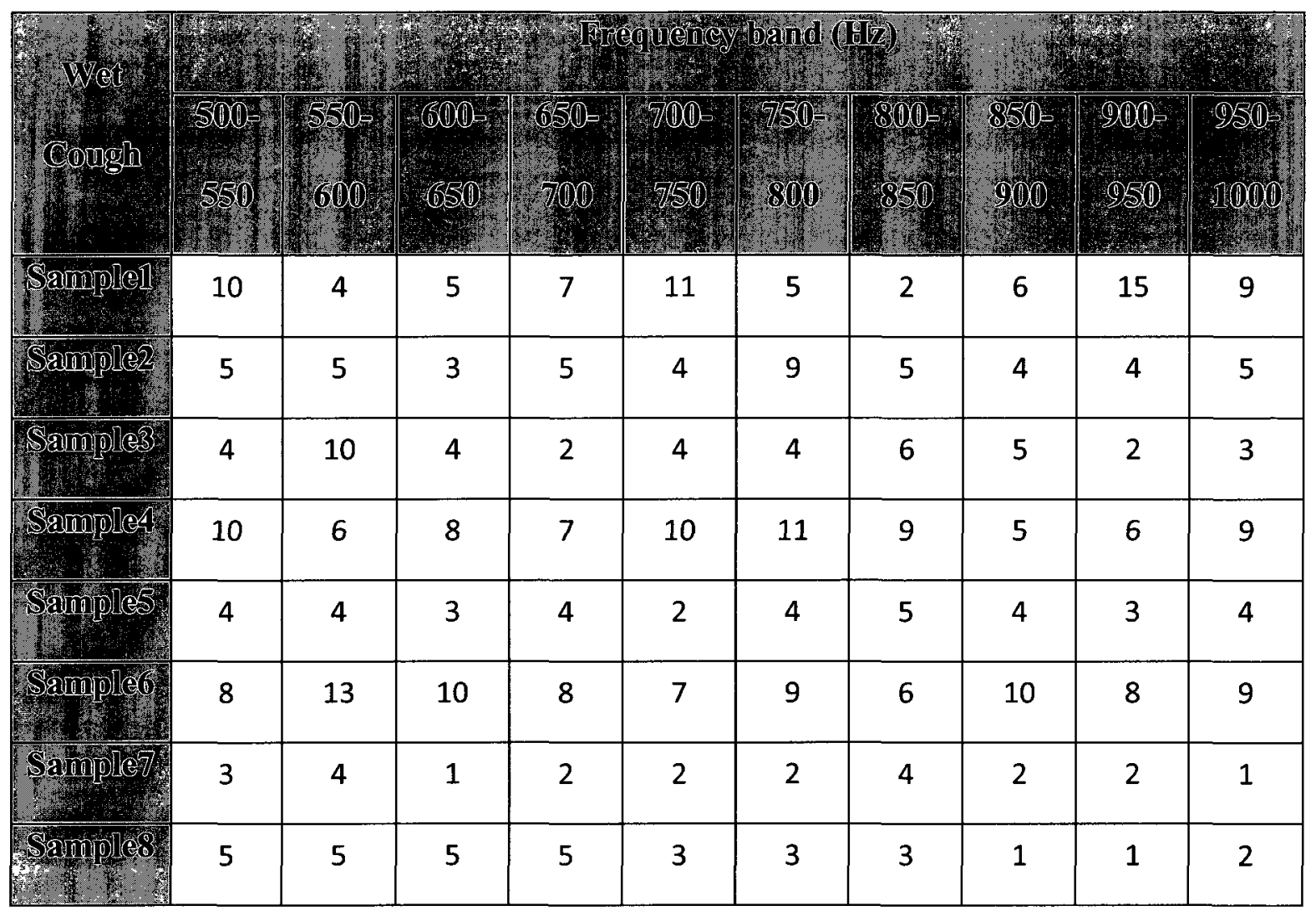

The number of peaks for three frequency bands $150-200 \mathrm{~Hz}, 200-250 \mathrm{~Hz}$, and 250 $300 \mathrm{~Hz}$ were plotted and are depicted in Figure 5.54, Figure 5.55, and Figure 5.56 respectively. In each frequency band there is an obvious separation between the two types of cough signals. However, the frequency band $200-250 \mathrm{~Hz}$ was selected as the most descriptive, since the most separation between dry and wet cough signals was observed. 


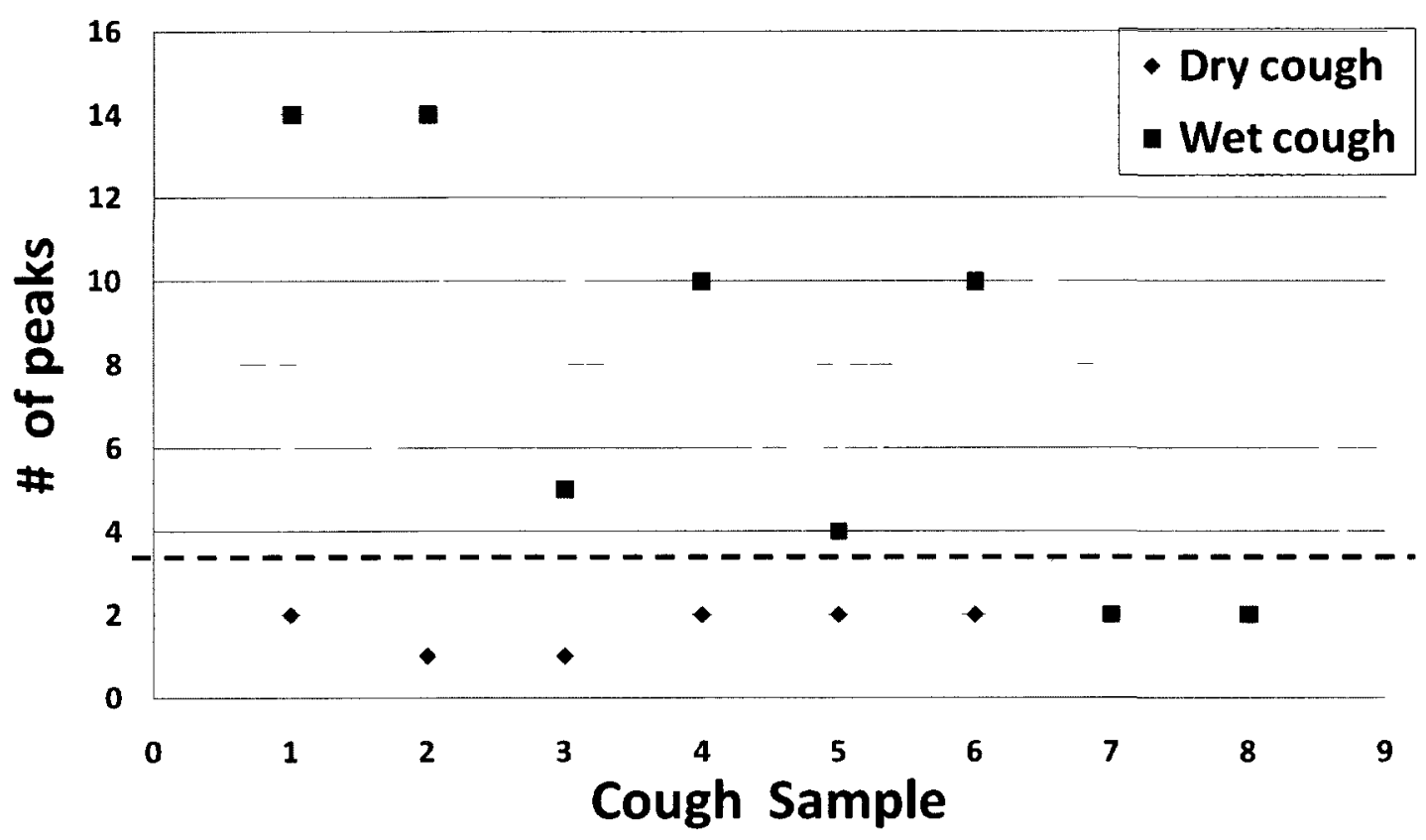

Figure 5.54: Number of peaks at 150-200 $\mathrm{Hz}$ for both dry and wet cough signals.

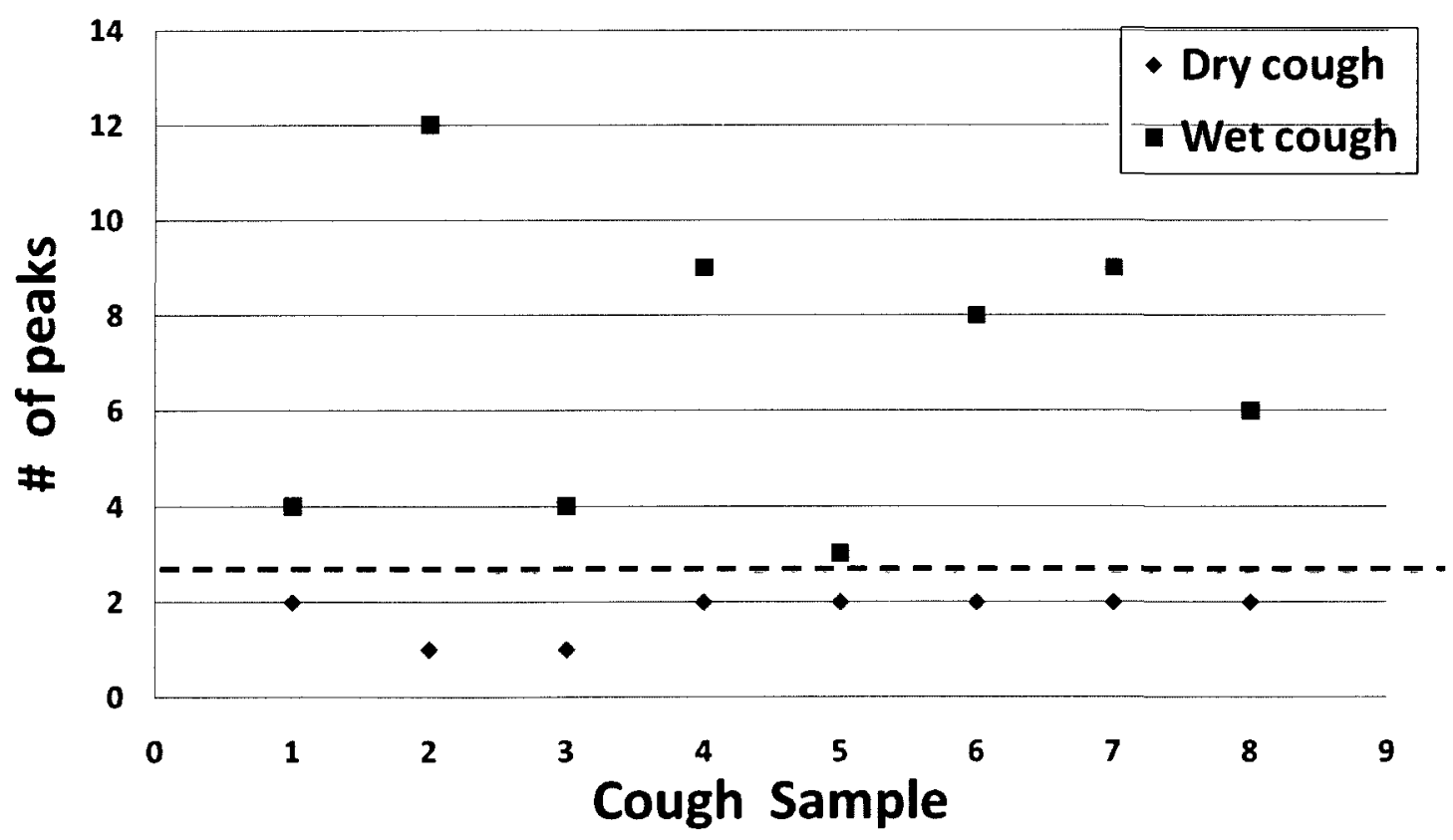

Figure 5.55: Number of peaks at $200-250 \mathrm{~Hz}$ for both dry and wet cough signals. 


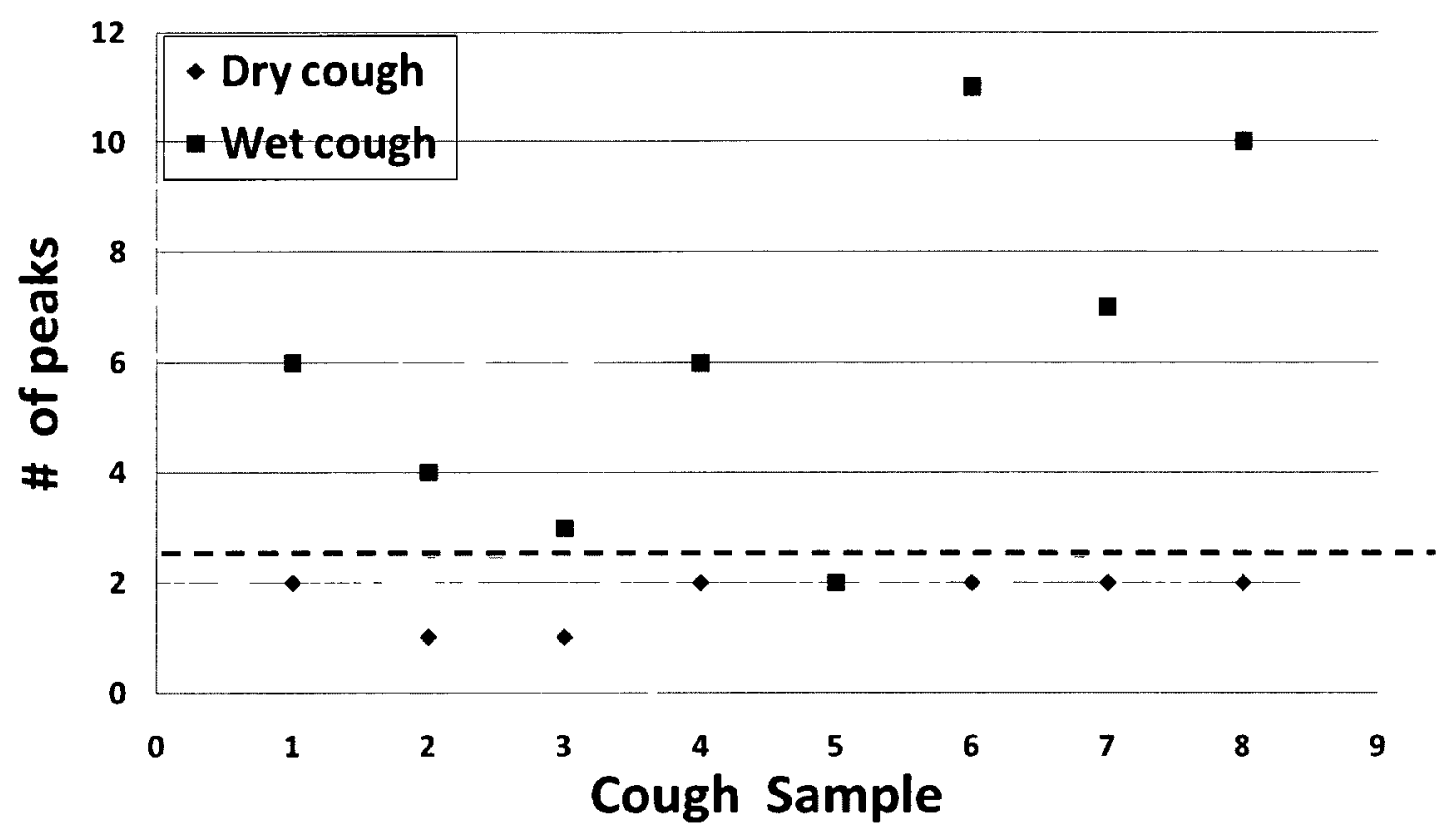

Figure 5.56: Number of peaks at $250-300 \mathrm{~Hz}$ for both dry and wet cough signals. 


\subsubsection{Results of Feature 1 Extraction Algorithm using Low-Pass Filter (LPF)}

The number of peaks for both dry and wet cough samples using a low-pass filter with different cut-off frequencies are listed in Table 10, Table 11, Table 12, and Table 13.

Table 10: Number of peaks of dry cough samples using LPF with cut-off frequency between $50-500 ~ H z$

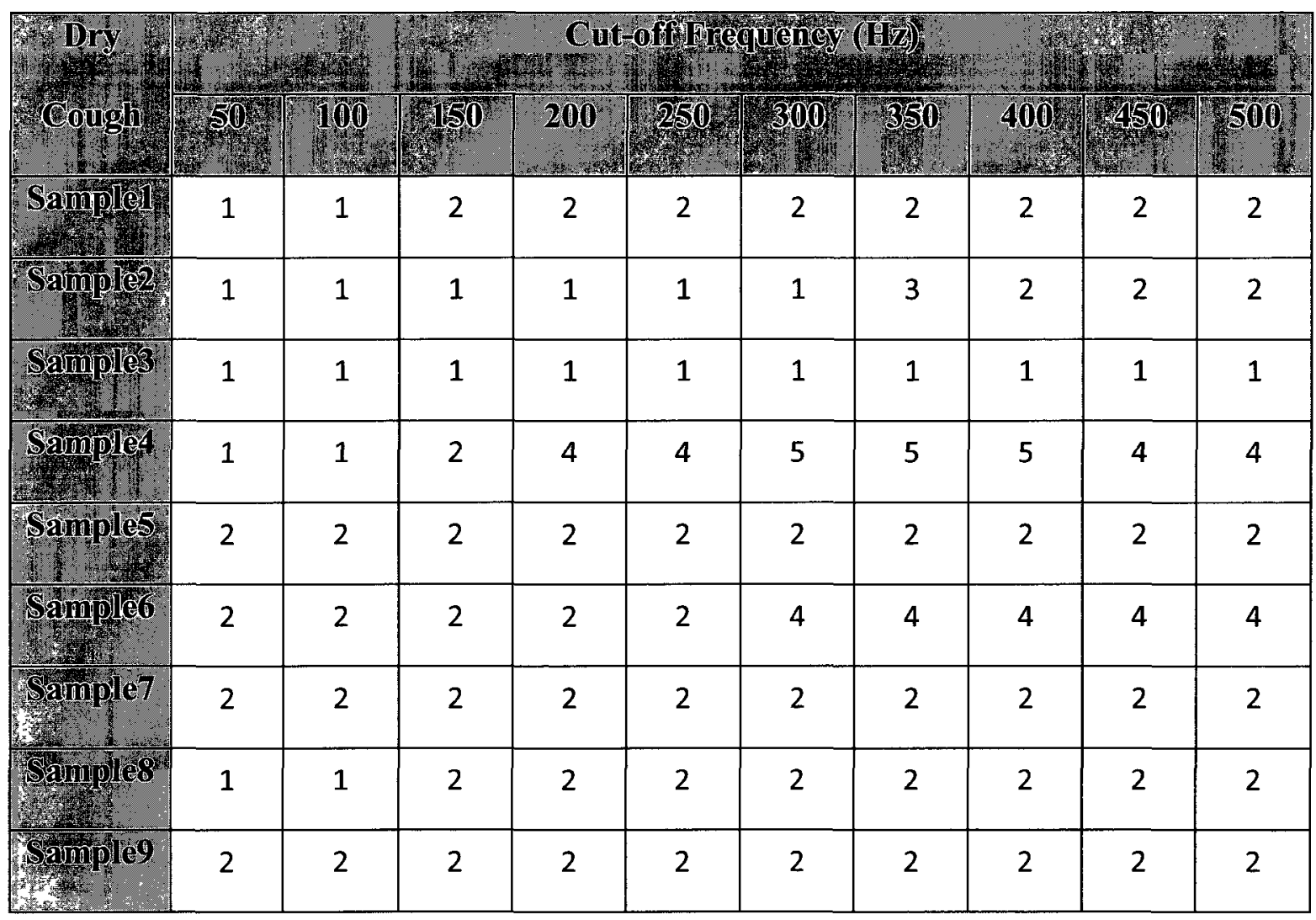


Table 11: Number of peaks of dry cough samples using LPF with cut-off frequency between 550-1000 Hz

\begin{tabular}{|c|c|c|c|c|c|c|c|c|c|c|}
\hline Woldy & $x^{2}$ & H' & (5) & 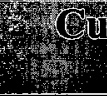 & $50 \mathrm{f} f \mathrm{ct}$ & Muengy & (3) & $4 \times$ & Pry & W. \\
\hline Congho & 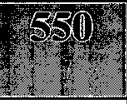 & 600 & 650 & 700 & 750 & 800 & 850 & OQDO & $\begin{array}{r}950 \\
8 \\
9\end{array}$ & (7000) \\
\hline Saropgle & 2 & 2 & 2 & 2 & 2 & 2 & 2 & 2 & 2 & 2 \\
\hline Sample2 & 2 & 2 & 2 & 2 & 2 & 2 & 2 & 3 & 2 & 2 \\
\hline Saropple 3 & 1 & 1 & 1 & 1 & 1 & 1 & 1 & 1 & 1 & 1 \\
\hline Samples & 5 & 4 & 5 & 5 & 4 & 4 & 4 & 4 & 4 & 4 \\
\hline Saromplest & 2 & 2 & 2 & 2 & 2 & 2 & 2 & 2 & 2 & 2 \\
\hline Saroppler & 5 & 4 & 5 & 4 & 5 & 4 & 4 & 4 & 5 & 5 \\
\hline Samplem & 5 & 2 & 2 & 2 & 2 & 2 & 2 & 2 & 2 & 3 \\
\hline Sampinger & 2 & 2 & 2 & 2 & 2 & 2 & 2 & 2 & 3 & 2 \\
\hline Sarogpheg & 2 & 2 & 2 & 4 & 8 & 7 & 6 & 5 & 6 & 6 \\
\hline
\end{tabular}


Table 12: Number of peaks of wet cough samples using LPF with cut-off frequency between 50-500 Hz

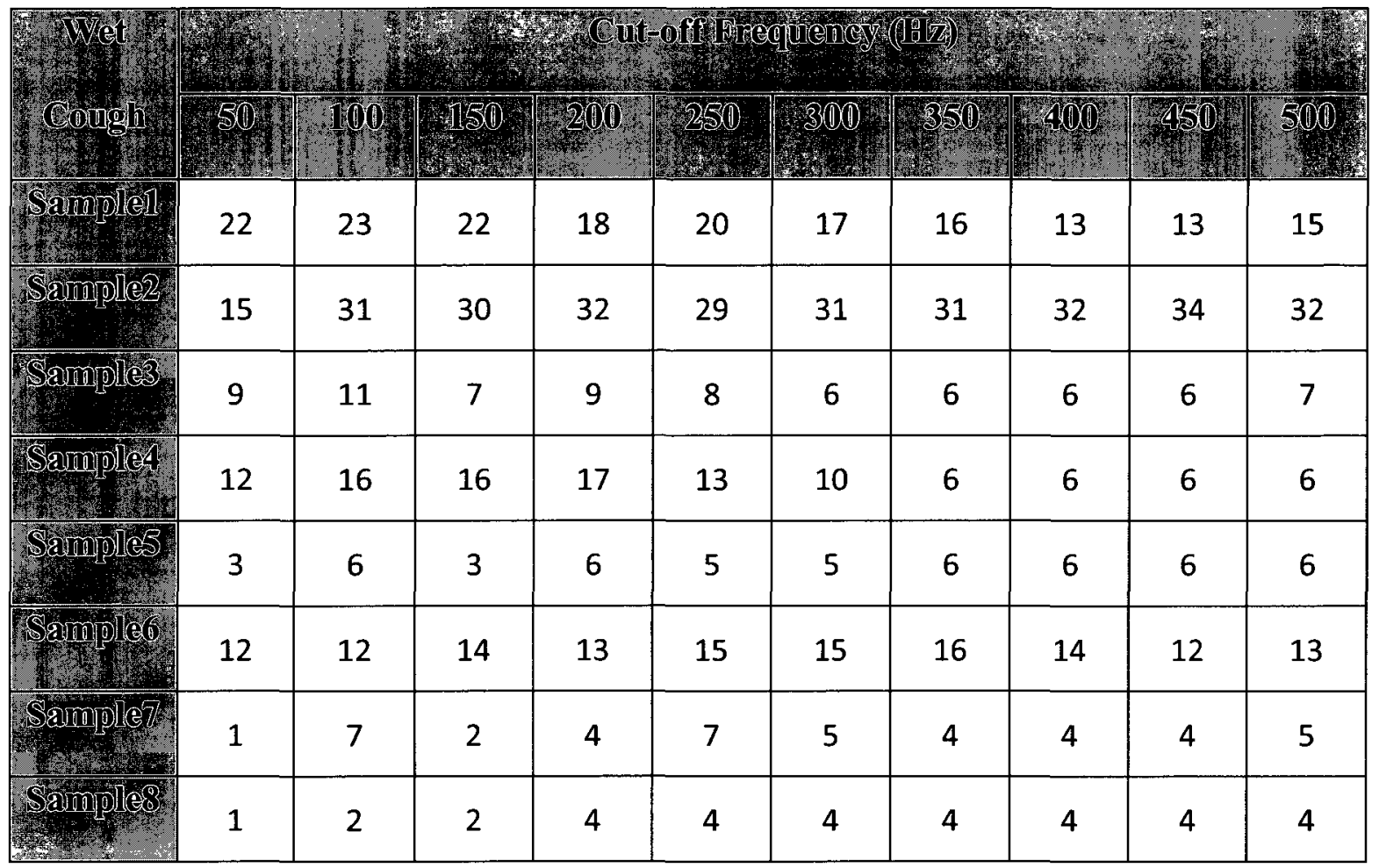

The results obtained for Feature 1 using the low-pass filter are similar to the results obtained with the band-pass filter at lower frequencies. The number of peaks for dry cough samples for most of the cut-off frequencies was between 1 and 3 . On the other hand, for most cases, the number of peaks for wet cough samples was more than 3 peaks. Hence, a clear separation was observed between the two types of cough sounds. 
Table 13: Number of peaks of wet cough samples using LPF with cut-off frequency between $550-1000 ~ H z$

\begin{tabular}{|c|c|c|c|c|c|c|c|c|c|c|}
\hline Whet & SW & We & Anges & 9.6ut & (a) for & pency & 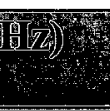 & W & 4 & (1) \\
\hline Cough & 55 & 60 & 650 & 700 & $\begin{array}{l}7550 \\
40\end{array}$ & 800 & 8850 & 9000 & 950 & 9000) \\
\hline Sampleit & 20 & 20 & 20 & 19 & 23 & 21 & 21 & 20 & 20 & 20 \\
\hline Sanopiled & 30 & 30 & 29 & 30 & 30 & 31 & 31 & 31 & 30 & 30 \\
\hline Sampoles & 3 & 3 & 3 & 4 & 4 & 3 & 4 & 4 & 5 & 4 \\
\hline Salonple4 & 6 & 6 & 6 & 6 & 6 & 6 & 6 & 6 & 6 & 6 \\
\hline Sa phples & 5 & 6 & 7 & 7 & 8 & 8 & 8 & 8 & 8 & 8 \\
\hline Sample6 & 12 & 11 & 11 & 11 & 12 & 13 & 13 & 12 & 13 & 12 \\
\hline Saropole & 5 & 7 & 5 & 5 & 6 & 4 & 3 & 3 & 3 & 3 \\
\hline Saropples & 4 & 4 & 3 & 4 & 4 & 4 & 4 & 4 & 4 & 4 \\
\hline
\end{tabular}

Figure 5.57, Figure 5.58, and Figure 5.59 show the number of peaks of dry and wet cough samples for three different low-pass cut-off frequencies. As it can be seen from the figures, a separation between dry and wet cough samples can be observed. 


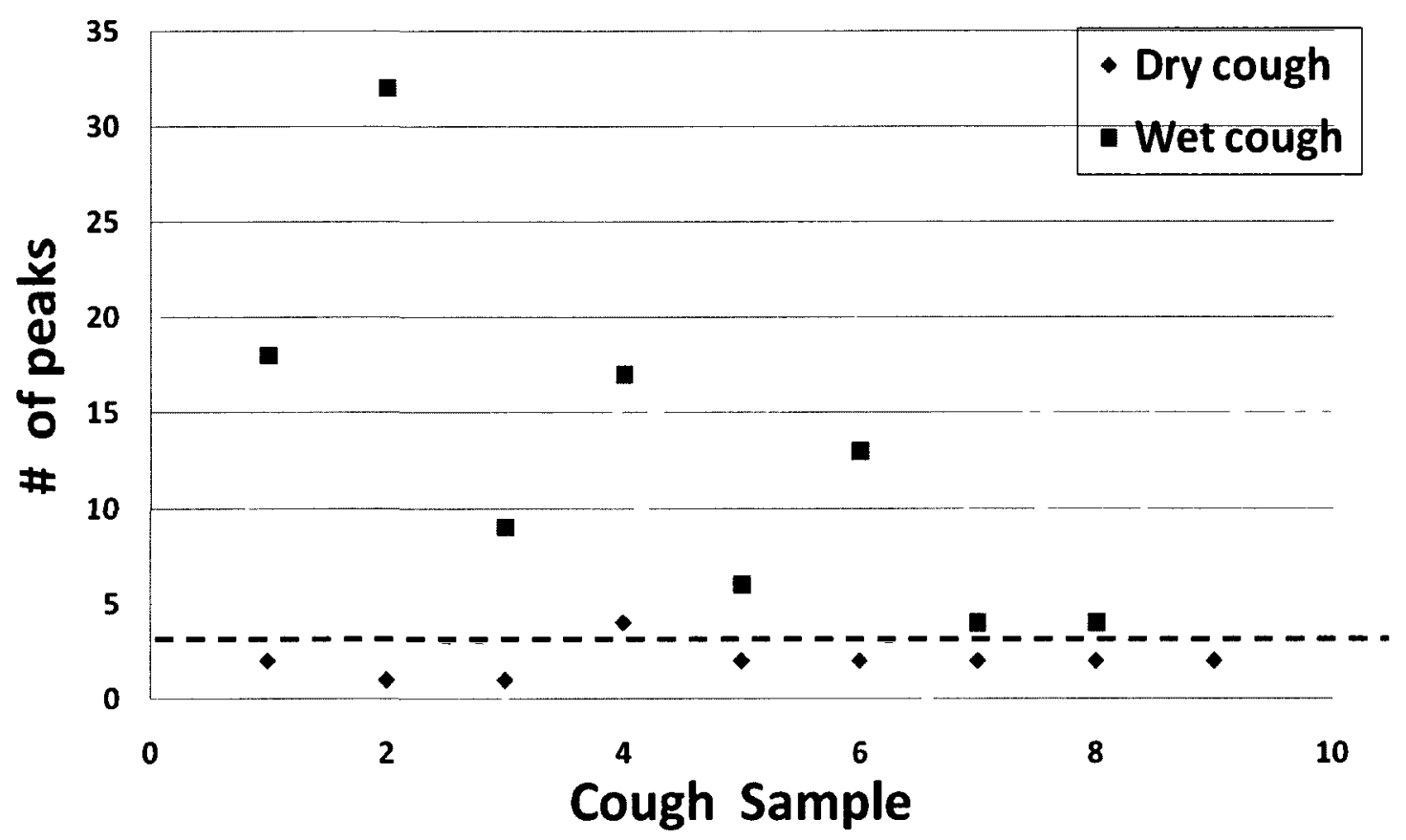

Figure 5.57: Number of peaks at $150-200 \mathrm{~Hz}$ for both dry and wet cough signals.

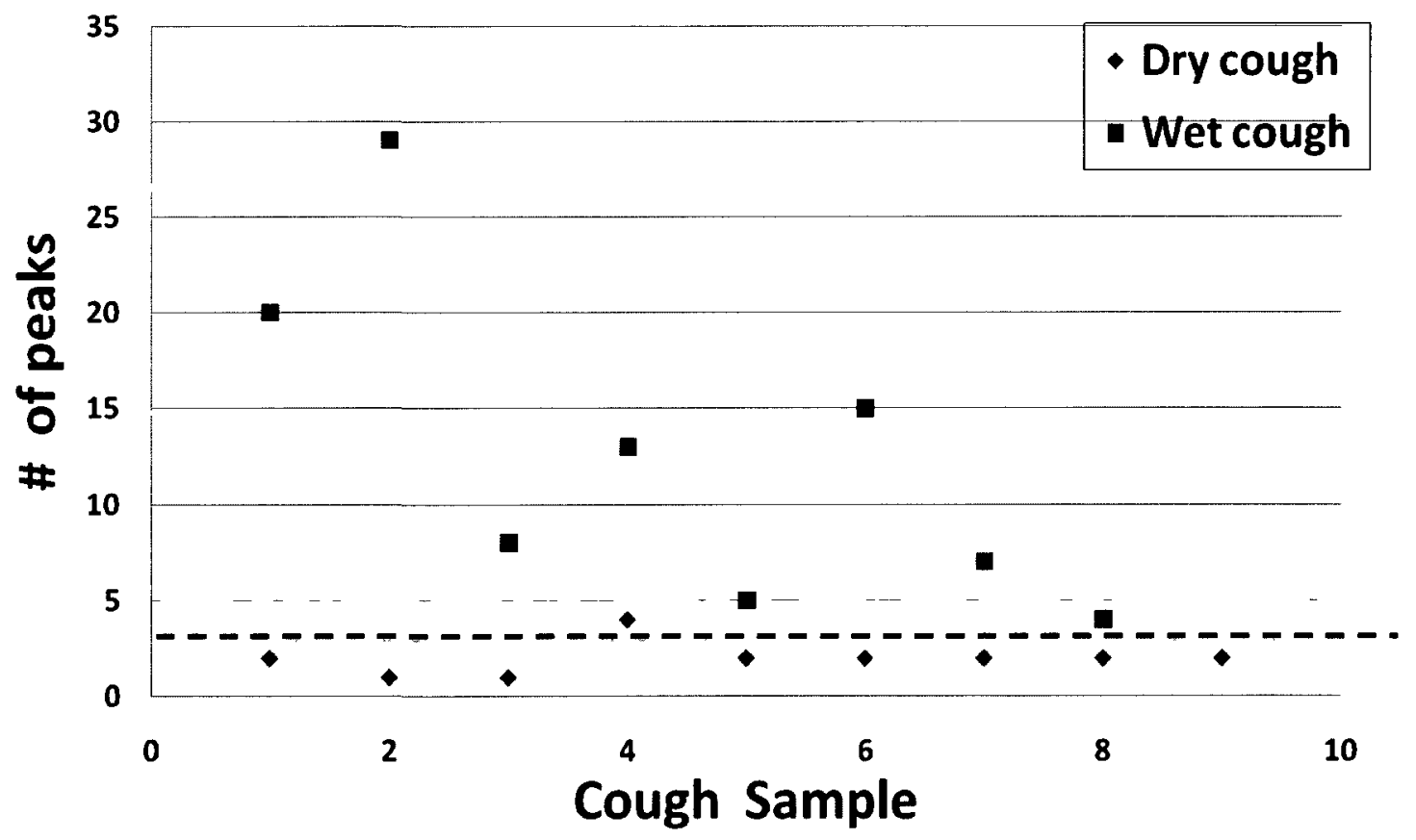

Figure 5.58: Number of peaks at 200-250 $\mathrm{Hz}$ for both dry and wet cough signals. 


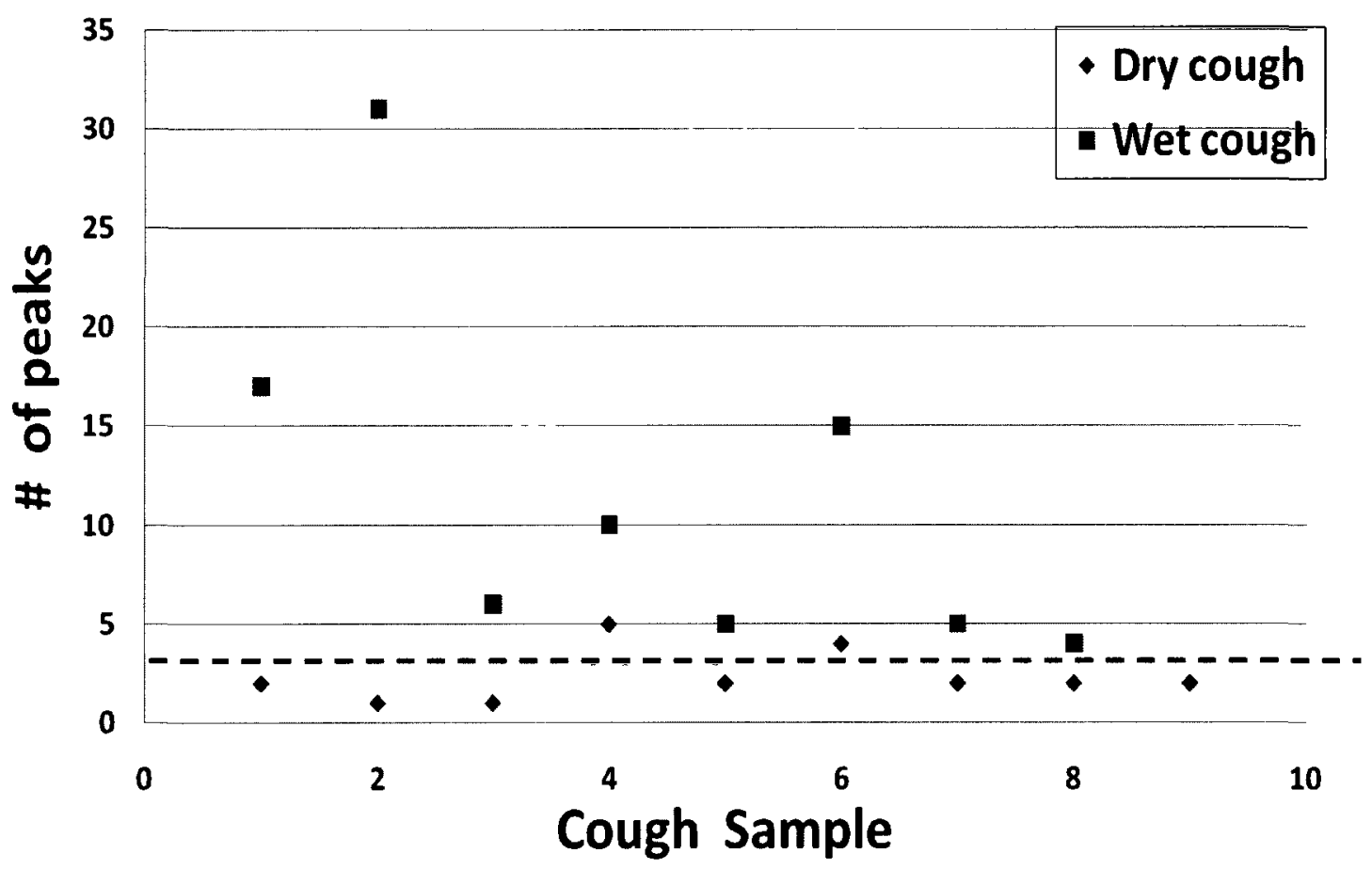

Figure 5.59: Number of peaks at $250-300 \mathrm{~Hz}$ for both dry and wet cough signals.

From the results obtained from using low-pass and band-pass filter to extract Feature 1, a clear separation between the two types of coughs is observed. In order to determine which type of filter is more suitable in extracting Feature 1, the misclassified cough samples for each frequency band were determined and depicted in Figure 5.60. The results show that BPF behaved better than LPF in most of the frequency bands. There were a few cases where the number of misclassified cough samples was equal for both low-pass and band-pass filter; however, for most of the cases, the band-pass filter showed a better result. 


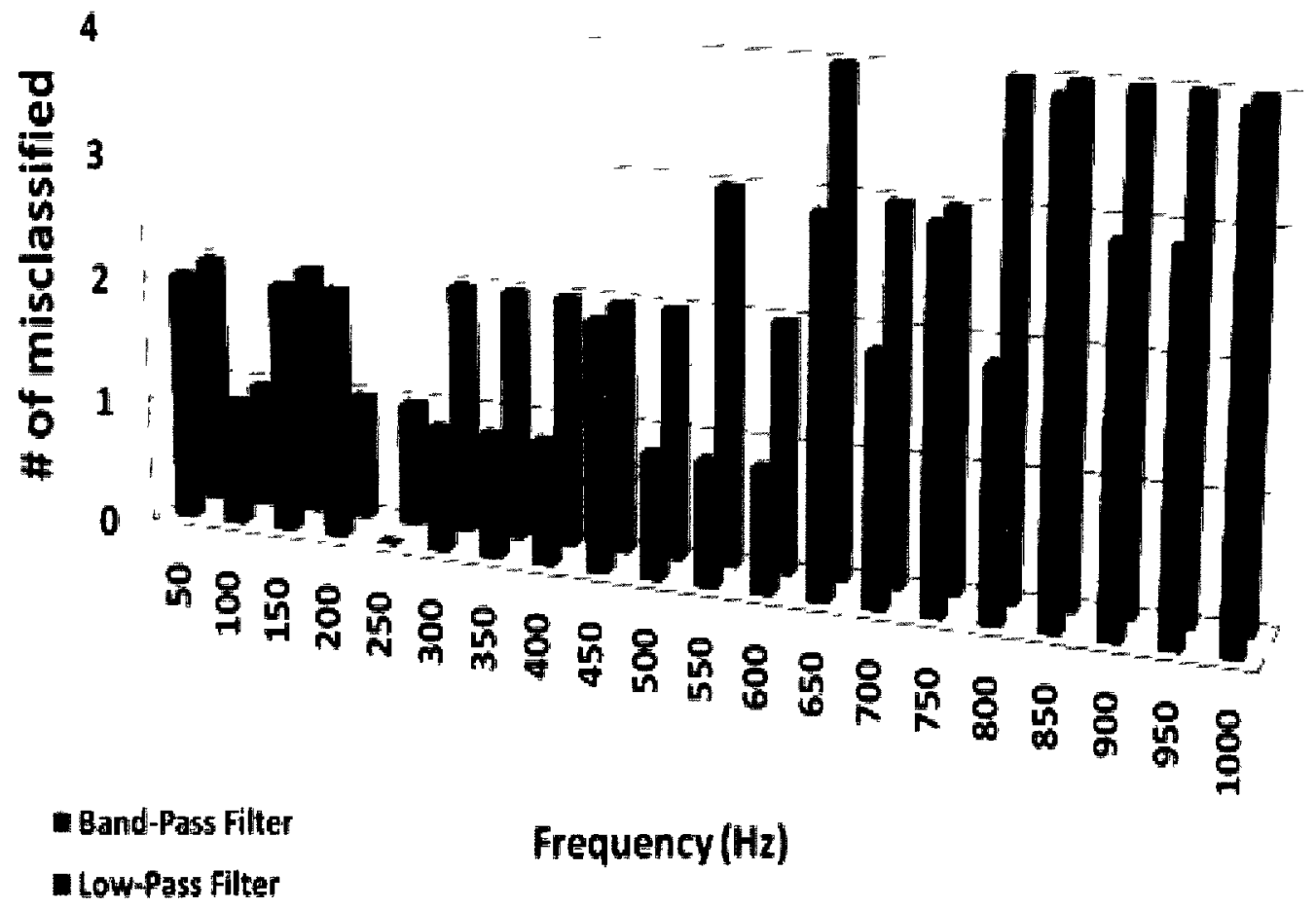

Figure 5.60: Misclassified cough samples

As can be seen from Figure 5.60, the frequency band $200-250 \mathrm{~Hz}$ for the band-pass filter results in $100 \%$ separation between dry and wet cough signals and therefore this frequency band was chosen as the most descriptive frequency band for Feature 1 extraction. 


\subsubsection{Results of Feature 2 Extraction Algorithm}

The second feature extraction algorithm was run for the same dry and wet cough signals as the previous section. The power ratio, $R p$, was calculated for both dry and wet cough signals and it is summarized in Table 14.

Table 14: $R p$ for both dry and wet cough samples

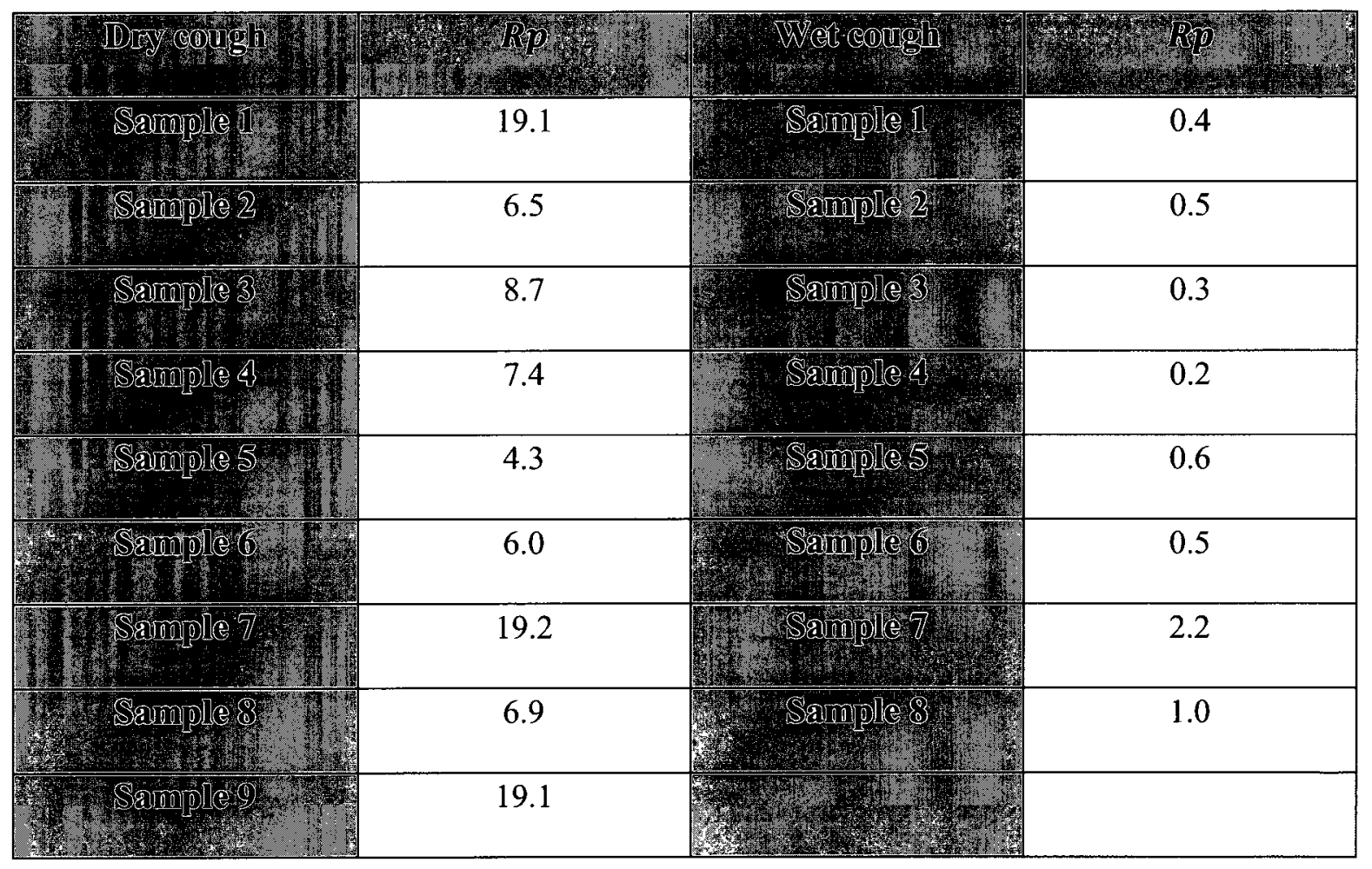

As can be seen from Table 14, the value of the $R p$ was observed to be $4<R p<20$ and $0<R p<2.5$ for dry and wet cough sounds approximately. Figure 5.61 shows the $R p$ for both dry and wet cough signals. From the figure, an obvious separation can be seen for the two types of cough signals. 


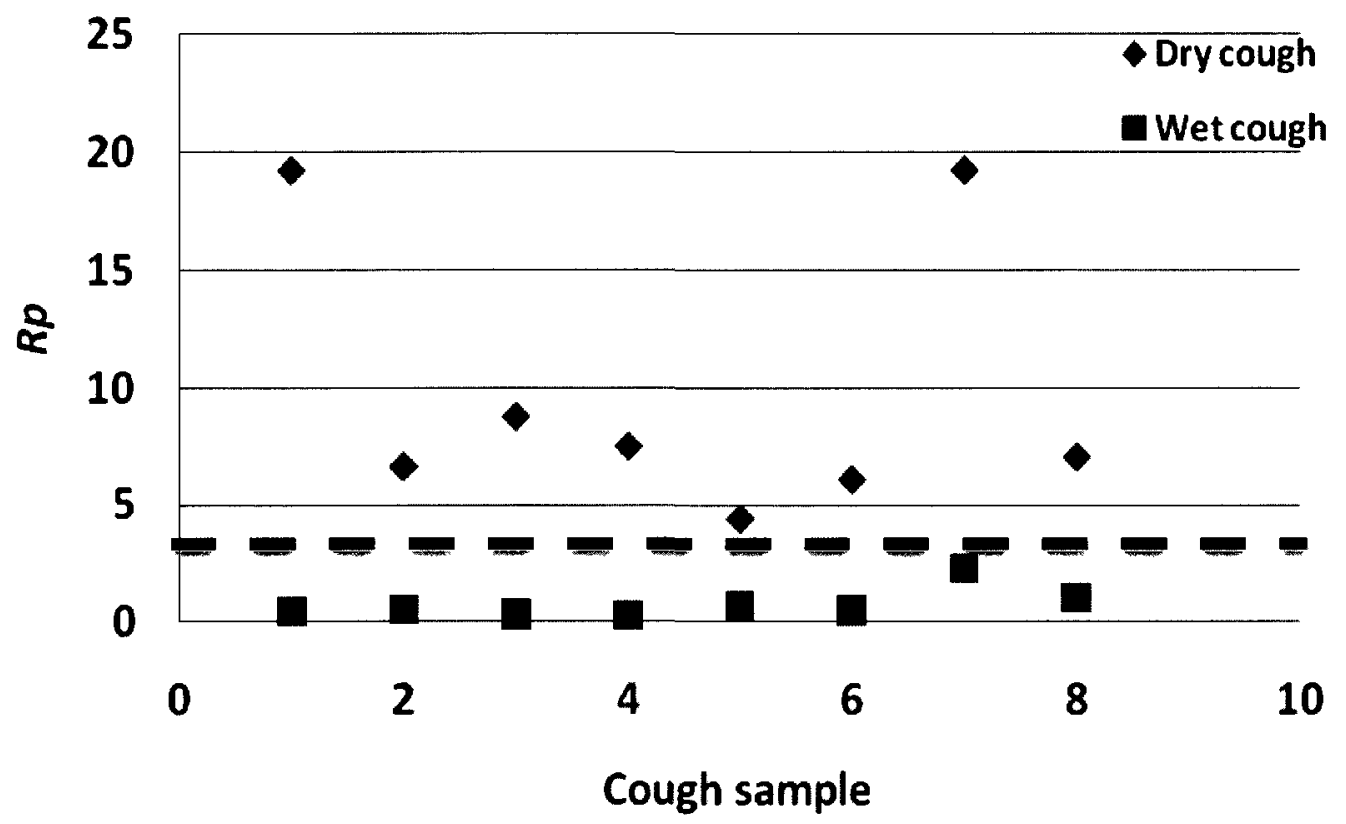

Figure 5.61: $R p$ for both dry and wet cough signals.

\subsubsection{Results of Feature 1 vs. Feature 2}

In this section, both Feature 1 and Feature 2 were used in order to differentiate between dry and wet cough signals. Figure 5.62 shows a clear separation between dry and wet cough signals using both Feature 1 and Feature 2. 


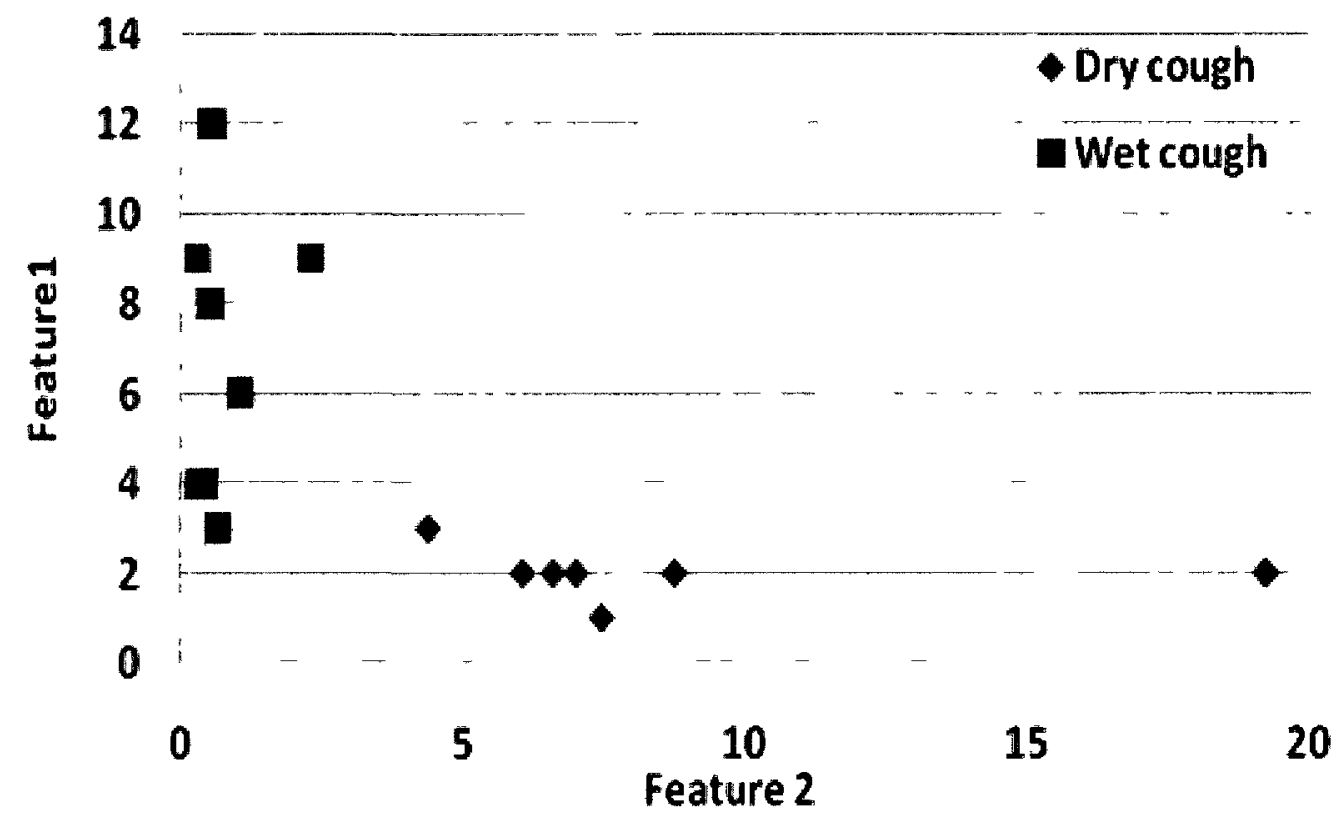

Figure 5.62: Separation of nine highly dry and eight highly wet cough sounds using Feature 1 and Feature 2.

\subsection{Discussion}

This chapter introduced two feature extraction algorithms in order to differentiate between two types of coughs, dry and wet. The first feature was based on the shape of the energy envelope of band-pass filtered cough signals. It was concluded that energy envelopes of dry cough signals have a specific shape. This observation, however, was not true for wet cough signals. The shape of the energy envelope of wet cough signals is 
mostly random and contains more peaks than dry cough signals. The frequency band 200$250 \mathrm{~Hz}$ was selected as the most descriptive frequency band. A clear separation line was observed between dry and wet cough samples (Figure 5.55).

The effect on Feature 1 of using a low-pass instead of band-pass filter was also explored. The performance of Feature 1 using low-pass filter was reasonable; however, the performance of the band-pass filter for the limited cough samples available was more satisfactory. Nevertheless, this does not mean that a low-pass filter cannot be used in computing Feature 1.

The second feature was based on the power ratio of two frequency bands of Phase 2 of the cough samples. This feature showed that the power ratio between the frequency band $1500-2250 \mathrm{~Hz}$ and the frequency band $0-750 \mathrm{~Hz}$ of Phase 2 of dry cough signals is greater than that of wet cough signals. Similar to Feature 1, a clear separation between the two types of coughs was observed for Feature 2 (Figure 5.61).

Using both Feature 1 and Feature 2, a clear separation is observed between the two types of cough samples (Figure 5.62). In Figure 5.62, blue markers show dry cough samples and red markers show wet coughs samples. As it can be seen from the figure, dry cough samples are spread along the $\mathrm{x}$-axis and wet cough samples along the $\mathrm{y}$-axis.

The cough samples used in this thesis were highly dry and highly wet cough samples as verified by clinicians. There are many cough samples, however, that fall between these two extremes. By taking into account cough samples with various degrees of wetness, the 
gap observed in Figure 5.62 between dry and wet cough samples decreases. Figure 5.63 illustrates the result of extracting Feature 1 and Feature 2 for cough samples that are not highly dry and highly wet. As seen from Figure 5.63, although there is still a clear separation between dry and wet cough sounds, the gap between the two types of coughs has decreased significantly. Further tests are needed to verify the results of Feature 1 and 2 for more samples.

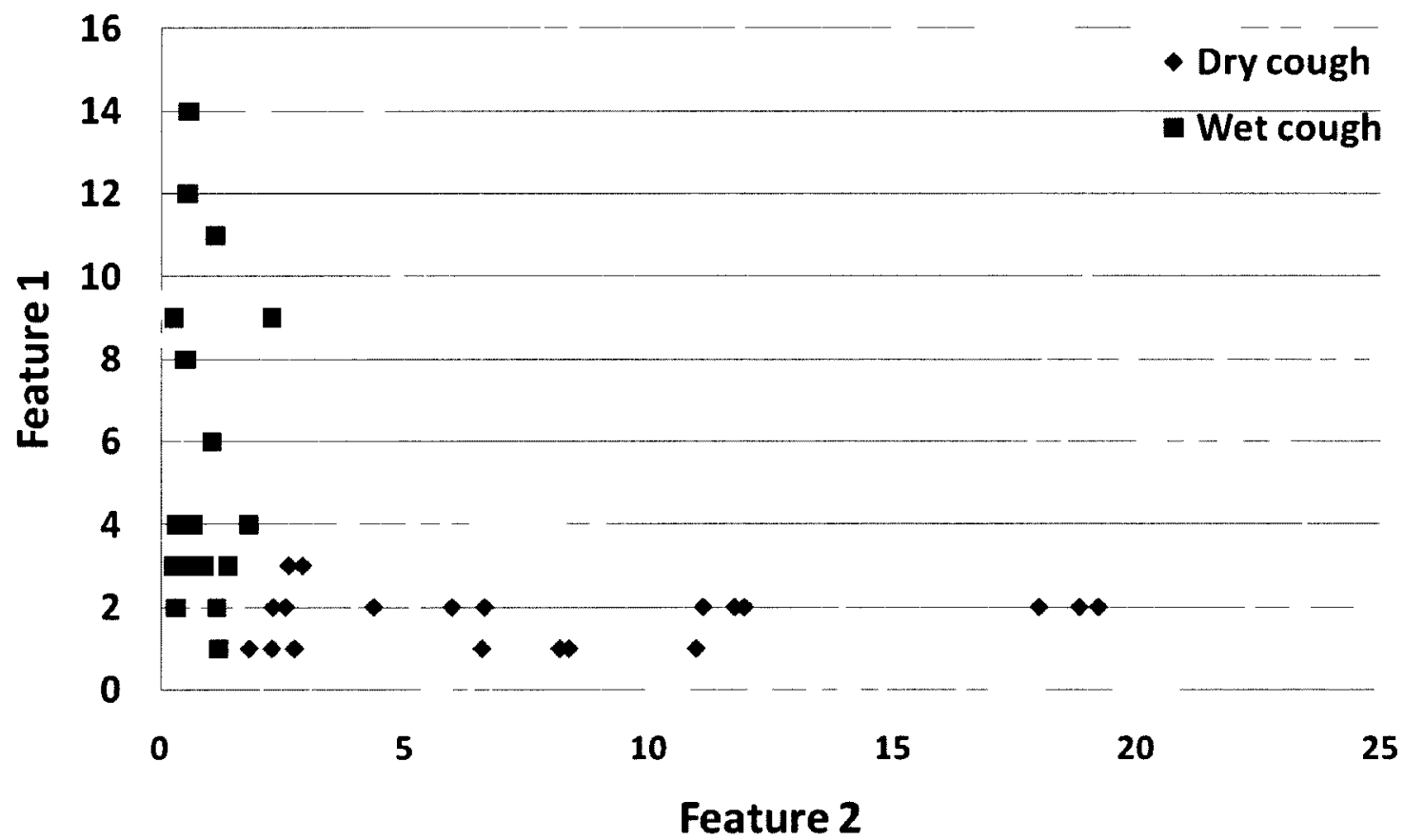

Figure 5.63: Separation of dry and wet cough samples using Feature 1 and Feature 2. 


\section{Chapter 6:}

\section{Graphical User Interface}

\subsection{Introduction}

This chapter introduces the Graphical User Interface (GUI) which was designed and implemented in order to help with the research conducted as part of this thesis.

Section 6.2 explains the design and functionality of the GUI in greater detail followed by conclusions in Section 6.3.

\subsection{Graphical User Interface Design}

A GUI was designed and implemented with Matlab in order to help with the extensive digital signal processing and analysis involved in the research. A snapshot of the GUI is depicted in Figure 6.1. The various functionalities of the GUI are grouped into three different panels, each of which will be explained in the following paragraphs. 
The first panel, distinguished with a blue background, performs basic functions such as opening and playing a file, zooming in and out of the displayed signal and playing the zoomed portion of the signal. Furthermore, this panel is responsible for performing various DSP related algorithms such as FFT and STFT and plotting them in a separate window, as depicted in Figure 6.2. Finally, this panel is capable of computing the length, total energy and the standard deviation of the input signal.

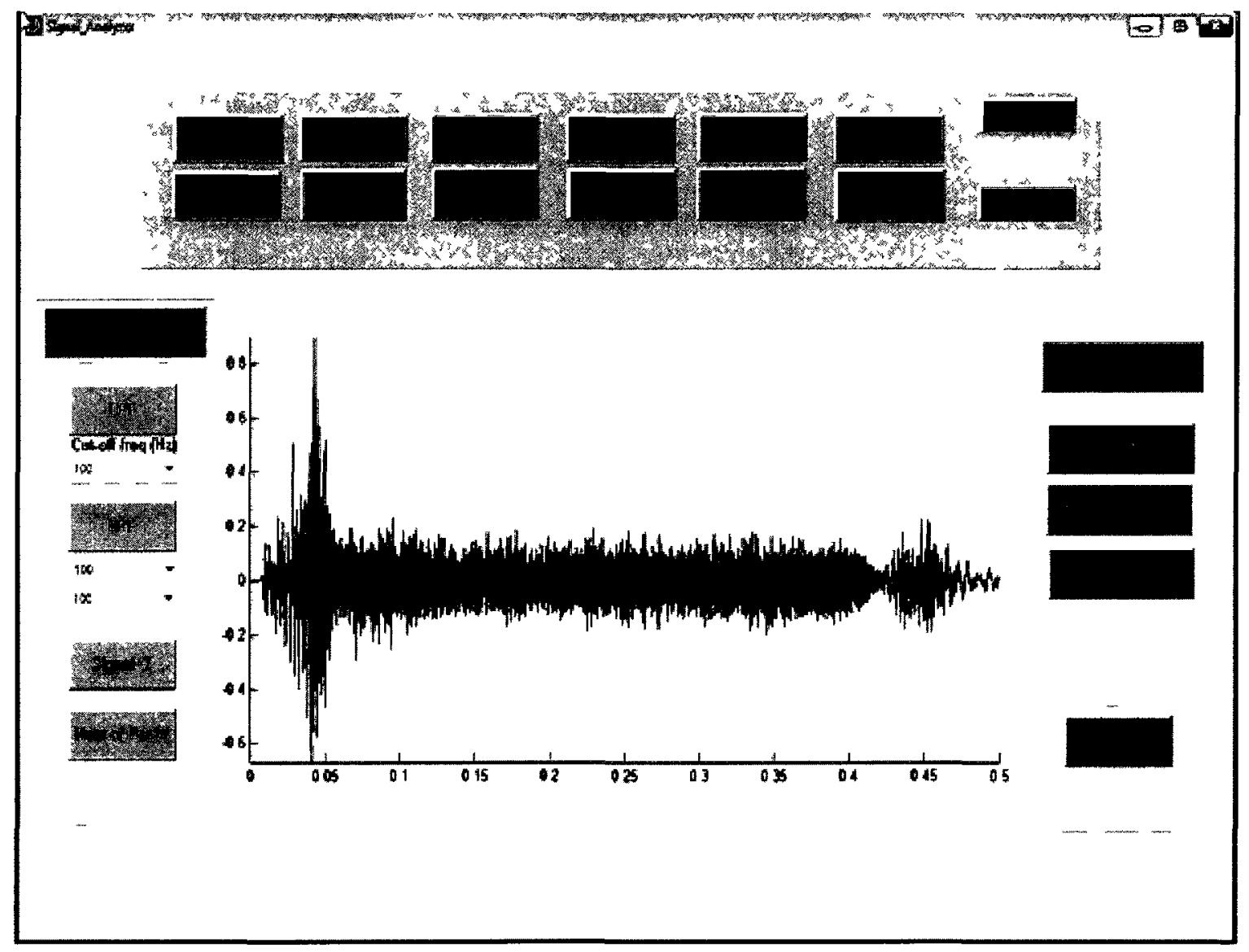

Figure 6.1: Graphical User Interface snapshot. 


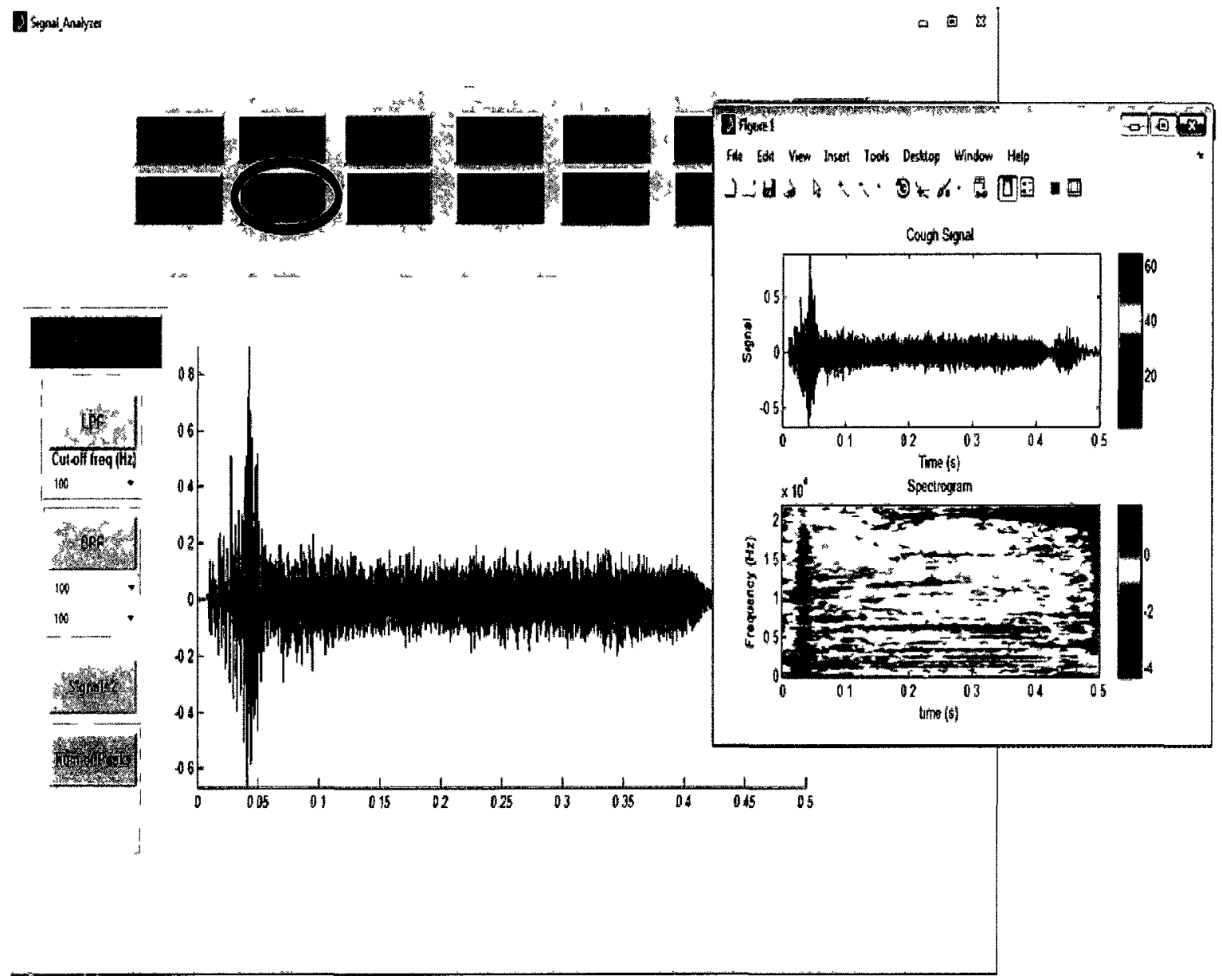

Figure 6.2: Show the STFT of the input signal in a separate figure.

The second panel, distinguished with a pink color theme, is responsible for performing the various signal processing algorithms used for computing the energy envelope variations (i.e. Feature 1), as depicted in Figure 6.3. This panel consists of 4 major buttons labelled "LPF", "BPF", "Signa1^2" and "Num of Peaks". The "LPF" button performs low-pass filter with various cut-off frequencies on the input signal. The cut-off frequencies are selectable via a drop-down menu. The "LPF" button also plots the 
result of the input signal passed through the low-pass filter in a new window. Similar to the "LPF" button, the "BPF" button performs band-pass filtering on the input signal with different cut-off frequencies selectable through a drop-down menu. Also, similar to the "LPF" button, a new figure is generated to show the band-pass filtered signal. The "Signal`2" button computes the square of the input signal which is the band-pass filtered signal generated by the "BFP" button. Finally, the "Num of Peaks" button calculates the number of peaks of the band-pass filtered signal and depicts it in a new figure as shown in Figure 6.3.

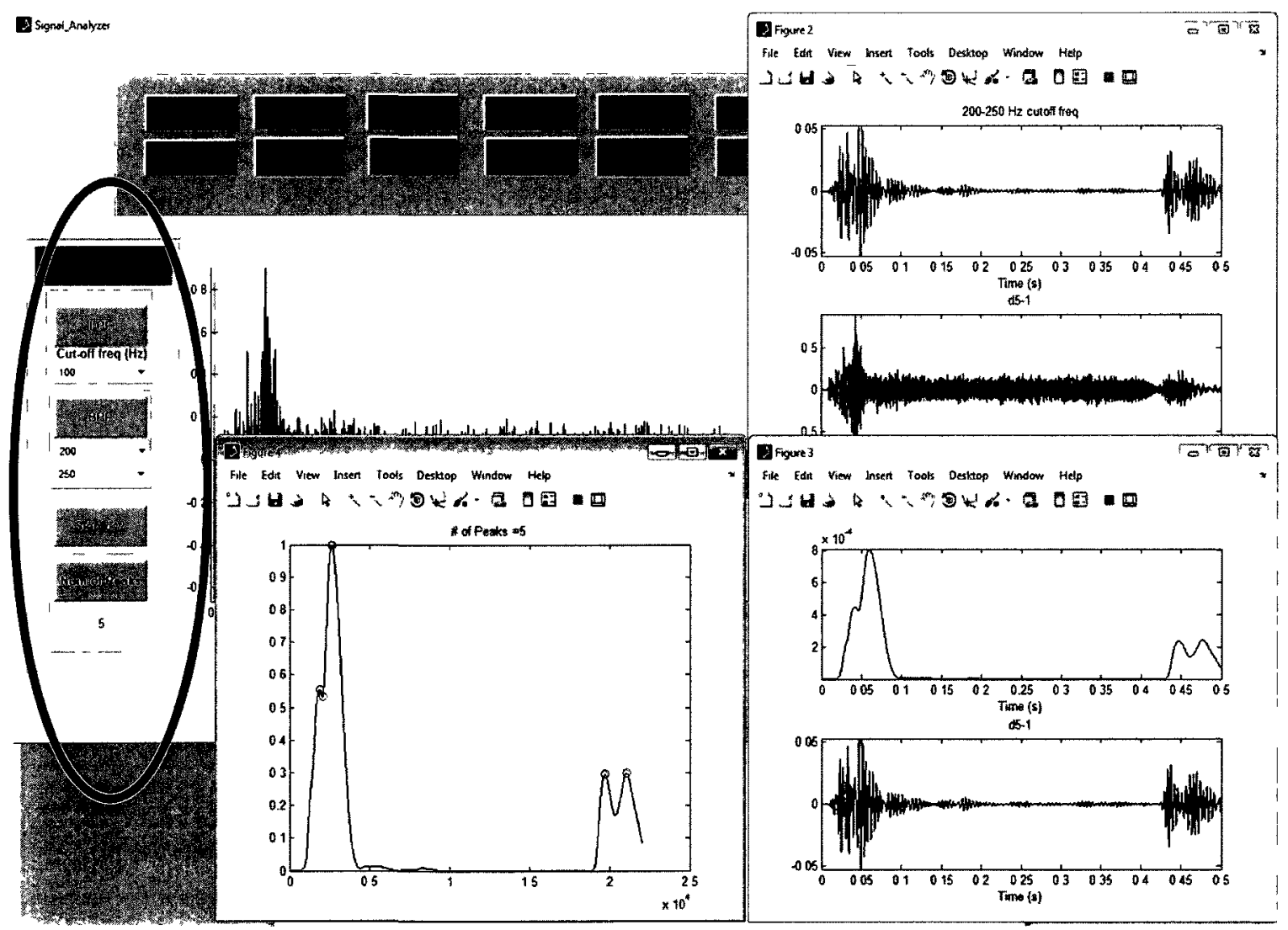

Figure 6.3: Functionality of the Feature 1 panel. 
Panel three, which has the color theme of green, can perform the signal processing algorithms related to Feature 2, such as power ratio estimation (depicted in Figure 6.4).

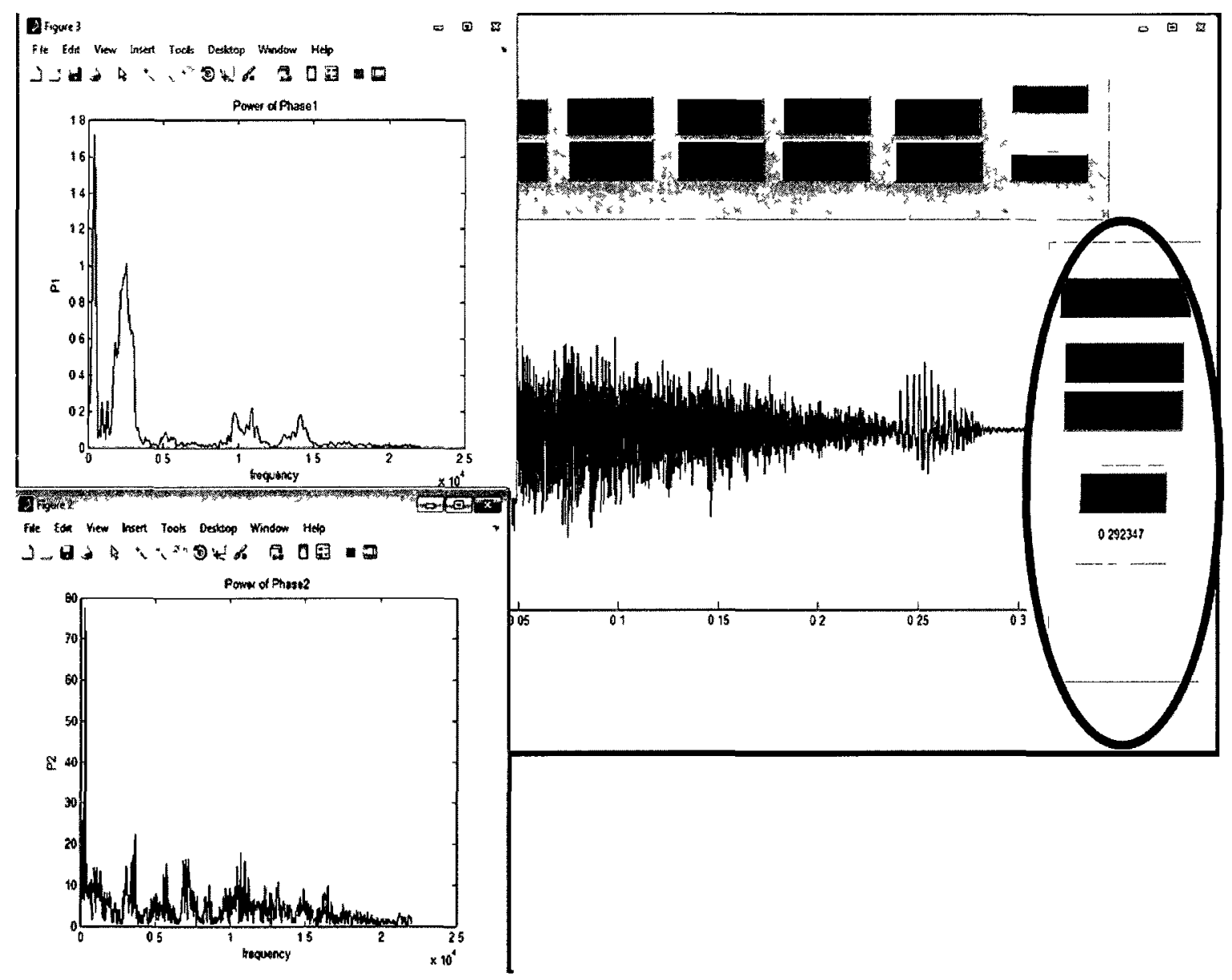

Figure 6.4: Functionality of the Feature 2 panel.

The power of each of the three phases of the input signal can be computed using the "Power of Phase1", "Power of Phase2" and "Power of Phase3" buttons respectively. The 
result of each button will be plotted in a new figure. The Rp of Feature 2 can be calculated using the "Rp" button.

\subsection{Conclusion}

This chapter explained the design and functions of the GUI tool that was designed in this thesis. The goal of this thesis is to find unique features which could distinguish between the two types of cough sounds. The purpose of the GUI was to provide a graphical user interface which contained all the functionalities required to conduct the research explained in this thesis. The GUI acted as a container that provided all the necessary signal analysis and signal processing algorithms in one convenient location. Furthermore, the GUI plotted the input signal after passing through various digital filters in order to provide visual feedback to the user and thus make the process easier and more efficient. 


\section{Chapter 7:}

\section{Conclusion}

\subsection{Thesis Conclusions and Contributions}

The main objective of this thesis was to differentiate between dry and wet cough. A novel Digital Signal Processing (DSP) algorithm was designed in order to extract useful clinical information from cough sounds.

A cough sound database was created using recorded cough sounds obtained from various sources. Cough signals were analyzed in both time and frequency domain. Two feature extraction algorithms were proposed in order to differentiate cough sounds into two classes, dry and wet. The first feature was the number of peaks of the energy envelope of the cough signal. The second feature was the power ratio of two frequency 
bands of the second phase of the cough signal. The feature extraction algorithms were verified with highly dry and highly wet cough sounds.

Using these two features, a significant differentiation between highly dry and highly wet cough sounds was observed, as depicted in Figure 5.62. The developed algorithms were trained and tested on a cough sound database which was verified by healthcare professionals.

The contributions that were made during this thesis research are the following:

Contribution 1: Collected cough sound data and built a database of dry and wet cough sounds. The nature of the coughs was verified by clinicians.

Contribution 2: Analyzed cough signals and identified unique features associated with dry and wet coughs.

Contribution 3: Proposed and implemented two feature extraction algorithms capable of differentiating dry and wet coughs. The first feature extraction algorithm is based on the number of peaks of the energy envelope of the cough signal. The second feature algorithm is based on the power ratio of the two frequency bands of the second phase of the cough signal. These features were tested using the collected cough sound database. This contribution was published in [10].

H. Chatrzarrin, A. Arcelus, R. Goubran, and F. Knoefel, "Feature extraction for the differentiation of dry and wet cough sounds," in Proc. IEEE International Symposium on Medical Measurements and Applications, Bari, Italy, May 2011, pp. 162-166. 
Contribution 4: Designed a GUI in Matlab in order to analyze cough signals in time and frequency domain. The GUI has various functionalities, such as computing the Fast Fourier Transform (FFT), Short-time Fourier Transform (STFT), signal energy and signal length of an input signal in addition to playing the input signal and extracting the two features.

\subsection{Suggested Future Work}

The following is the list of future work:

- Collect data from real patients with different respiratory diseases.

- Rate the cough sounds that fall in between the ranges of wet and dry coughs.

- Work towards detecting changes in cough sound from chronic cough to infected cough.

- Add more feature extraction algorithms to have a better separation between dry and wet cough sounds.

- Add more features to the GUI for future researches, to show clinical information such as the frequency of cough, intensity and nature of the cough.

- Take into account background noise and reverberation, which might impact the performance of the cough monitoring systems. 


\section{References}

[1] S. Matos, S.S. Birring, I.D. Pavord, and D.H. Evans, "An automated system for 24-h monitoring of cough frequency: The leicester cough monitor," IEEE Engineering in Medicine and Biology Society, vol. 54, no. 8, pp. 1472-1479, Aug. 2007.

[2] S.J. Barry, A.D. Dane, A.H. Morice, and A.D. Walmsley, "The automatic recognition and counting of cough," Cough, vol.2, Sep. 2006.

[3] D.T.H. Lai, R.K. Begg, and M. Palaniswami, "Computational intelligence in gait research: a perspective on current applications and future challenges," IEEE Transactions on Information Technology in Biomedicine, vol. 13, no. 5, pp. 687-702, Sep. 2009.

[4] M. H. Jones, A. Arcelus, R. A. Goubran, and F. Knoefel, "A pressure sensitive home environment," in Proc. IEEE International Workshop on Haptic Audio Visual Environments Application, Ottawa, ON, Canada, Nov. 2006, pp. 10-14.

[5] A. Arcelus, M.H. Jones, R. Goubran, and F. Knoefel, "Integration of smart home technologies in a health monitoring system for the elderly," in Proc. Advanced Information Networking and Application Workshops, Niagara Falls, Canada, May 2007, pp. 820-825.

[6] A. Arcelus, M. Holtzman, R. Goubran, H. Sveistrup, P. Guitard, and F. Knoefel, "Analysis of commode grab bar usage for the monitoring of older adults in the smart home environment," in Proc. $31^{\text {st }}$ International Conference of the IEEE Engineering in Medicine and Biology Society, Minneapolis, MN, USA, 2009, pp. 6155-6158.

[7] M. Guennoun, and K. El-Khatib, "Securing medical data in smart homes," in Proc. IEEE International Workshop on Medical Measurements and Applications, Cetraro, Italy, May 2009, pp. 104-107.

[8] I. Bosznai, F. Ender, and H. Santha, "Web server based remote health monitoring system," in Proc. $32^{\text {nd }}$ International Spring Seminar on Electronic Technology, Brno, Czech Republic, May 2009, pp. 16. 
[9] R.J. Jaeger, J.P. Szidon, and P.J Doucette, "Instrumentation for measuring frequency of cough," in Proc. The $17^{\text {th }}$ Conference of the IEEE Engineering in Medicine and Biology Society, Montreal, Canada, Sep. 1995, pp. 1645-1646.

[10]H. Chatrzarrin, A. Arcelus, R. Goubran, and F. Knoefel, "Feature extraction for the differentiation of dry and wet cough sounds," in Proc. IEEE International Symposium on Medical Measurements and Applications, Bari, Italy, May 2011, pp. 162-166.

[11] G.A. Fontana, "Before we get started: what is a cough?," lung, supp. 1, s3-6, Oct. 2008.

[12] A. Molassiotis, J.A. Smith, M.I. Bennett, F. Blackhall, D. Taylor, B. Zavery, A. Harle, R. Booton, E.M. Rankin, M. Lloyd-Williams, A.H. Morice, "Clinical expert guidelines for the management of cough in lung cancer: report of a UK task group on cough," Cough, vol. 9, 2010.

[13] M. Vernon, N.K. Leidy, A. Nacson, and L. Nelsen, "Measuring cough severity: perspective from the literature and from patients with chronic cough," Cough, vol. 5, 2009.

[14] N.M. Ryan, A.E. Vertigan, S. Bone, and P.G Gibson, "Cough reflex sensitivity improves with speech language pathology management of refractory chronic cough," Cough, vol. 6, 2010.

[15]K.F. Chung, D. Bolser, P. Davenport, G. Fontana, A. Morice, J. Widdicombe, "Semantic and types of cough," Pulmonary Pharmacology and Therapeutics, vol. 22, issue 2, pp. 139-142, Apr. 2009.

[16] J. Korpas, J. Sadlonova, and M. Vrabec, "Analysis of the cough sound: an overview," Pulmonary Pharmacology, vol. 9, pp. 261-268, Oct. 1996.

[17] A. Murata, Y. Taniguchi, Y. Hashimoto, Y. Kaneko, Y. Takasaki, and S. Kudoh, "Discrimination of productive and non-productive cough by sound analysis," Internal Medicine, vol. 37, no. 9, pp. 732735, Sep. 1998.

[18]P. Piirila and A.R. Sovijarvi, "Differences in acoustic and dynamic characteristics of spontaneous cough in pulmonary diseases," Chest, vol. 96, pp. 46-53, Jul. 1998.

[19] Y. Hashimoto, A. Murata, M. Mikami, S. Nakamura, E. Yamanaka, and S. Kudoh, "Influence of the rheological properties of airways mucus on cough sound generation," Respiratory, vol. 8, pp. 45-51, Mar. 2003.

[20] J.A. Smith, H.L. Ashurst, S. Jack, A.A. Woodcock, and J.E. Earis, "The description of cough sounds by healthcare professionals," Cough, vol. 2, Jan. 2006.

[21] A.V. Hirtum and D. Berckmans, "Assessing the sound of cough towards vocality," Meical Engineering and Physics, vol. 24, pp. 535-540, Sep. 2002. 
[22] S. Farrari, M. Silva, M. Guarino, J.M. Aerts, and D. Berckmans, "Cough sound analysis to identify respiratory infection in pigs," Computer and Electronics in Agriculture, vol. 64, pp. 318-325, Dec. 2008.

[23]C. Bianchi and P. Baiardi, "Cough peak flows: standard values for children and adolescents," American Journal of Physical Medicine and Rehabilitation, vol. 87, pp. 461-467, Jun. 2008.

[24] G.E. Murty, M.C.F. Smith, and P. Lancaster, "Cough intensity in the laryngectomee," Clinical Otalaryngology and Allied Sciences, vol. 16, issue 1, Jul. 1989.

[25] A.H. Morice, G.A. Fontana, M.G. Belvisi, S.S. Birring, K.F. Chung, P.V. Dicpinigaitis, J.A. Kastelik, L.P. McGarvey, J.A. Smith, M. Tatar, and J. Widdicombe, "ERS guidelines on the assessment of cough," European Respiratory Journal, vol. 29, pp. 1256-1276, Jun. 2007.

[26]Y. Li, Z. Zeng, M. Popescu, and K.C. Ho, "Acoustic fall detection using a circular microphone array," in Proc. $32^{\text {nd }}$ International Spring Seminar on Electronic Technology, Buenos Aires, Argentina, Aug. 2010, pp. 2242-2245.

[27] S. Huq, and Zahra Moussavi, "Automatic breathe phase detection using only tracheal sounds," in Proc. $32^{\text {nd }}$ Annual International Conference on Engineering in Medicine and Biology Society (EMBC), Buenos Aires, Argentina, Sep. 2010, pp. 272-275.

[28] C.R. Woolf and A. Rosenberg, "Objective assessment of cough suppressants under clinical condition using a tape recorder system," Thorax, vol. 19, pp. 125-130, Mar. 1964.

[29] W. Ser, T.T. Zhang, J. Yu, and J. Zhang, "Detection of wheezes using a wearable distributed array of microphones," in Proc. $6^{\text {th }}$ Intrenational Workshop on Wearable and Implantable Body Sensor Networks, Berkely, CA, Jun. 2009, pp. 296-300.

[30]Y. Hata, S. Kobashi, H. Yamaguchi, O. Ishikawa, N. Tsuchiya, and H. Nakajima, "Human health monitoring system of systems by non-contacted sensors," in Proc. . IEEE Workshop on Robotic Intelligence in Informationally Structured Space (RiiSS), May 2009.

[31] Y. Hata, S. Kobashi, K. Kuramoto, H. Nakajima, "Home care system for aging people confined to bed by detached sensor network," in Proc. IEEE Workshop on Robotic Intelligence in Informationally Structured Space (RiiSS), Paris, France, Apr. 2011.

[32] S. Matos, S.S. Birring, I.D. Pavord, and D.H. Evans, "Detection of cough signals in continuous audio recording using hidden markov models," IEEE Transaction on Biomedical Engineering, vol. 53, no. 6, Jun. 2006. 
[33] S.H. Shin, T. Hashimoto, and S. Hatano, "Automatic detection system for cough sounds as a symptom of abnormal health condition," IEEE Transaction on Information Technology in Biomedicine, vol. 13, no. 4, Jul. 2009.

[34] A.A. Abaza, J.B. Day, J.S. Reynolds, A.M. Mahmoud, W.T. Goldsmith, W.G. McKinney, E.L. Petsonk, and D.G. Frazer, "Classification of voluntary cough sounds and airflow patterns for detecting abnormal pulmonary function," Cough, vol. 5, Nov. 2009.

[35] A.V. Hirtum, and D. Berckmans, "Automated recognition of spontaneous versus voluntary cough," Medical Engineering and Physics, vol. 24, pp. 541-545, May. 2002.

[36] J. Martinek, M. Tatar, M. Javorka, "Distinction between voluntary cough sound and speech in voluntarees by spectral and complexity analysis," Journal of Physiology and Pharmacology, vol. 59, pp. 433-440, Dec. 2008.

[37] C.H. Lu and L.C Fu, "Robust location-aware activity recognition using wireless sensor network in an attentive home," IEEE Transaction on Automation Science and Engineering, vol. 6, no. 4, pp. 598-609, Oct. 2009.

[38]H.H. Kim, K.N. Ha, S. Lee, and K.C. Lee, "Resident location-recognition algorithm using a Bayesian classifier in the PIR sensor-based indoor location-aware system," IEEE Transaction on Systems, Man, and Cybernetics Part C: Applications and Reviews, vol. 39, no. 2, pp. 240-245, Mar. 2009.

[39] M. Vacher, N. Guirand, J.F. Serignat, A. Fleury, and N. Noury, "Speech recognition in a smart home: some experiments for telemonitoring," in Proc. $5^{\text {th }}$ Conference Speech Technology and HumanComputer dialogue, Constanta, Romania, Jun. 2009, pp. 1-10.

[40] T.T. Zhang, W. Ser, G.Y.T. Daniel, J. Zhang, J. Yu, C. Chua, and I.M. Louis, "Sound based heart rate monitoring for wearable systems," in Proc. Conference on Body Sensor Networks (BSN), Singapore, Jun. 2010, pp. 139-143.

[41]H. Andoh, T. Ishikawa, K. Kobayashi, K. Kobayashi, K. Watanabe, and T. Nakamura, "Home health monitoring system in the sleep," in Proc. SICE Annual Conference, Fukui, Japan, Aug. 2003, vol. 3 pp. 1223-1226.

[42] M. Mosmondor, I. Benc, S. Desic, and A. Grguric, "A feasibility study for the integration of a remote patient monitoring solution with electronic health record system," in Proc. $33^{\text {rd }}$ International Convention on Information and Communication Technology, Electronics and Microelectronics, Opatija, Croatia, May 2010, pp. 360-366.

[43]A. Fleury, M. Vacher, and N. Noury, "SVM-based multimodal classification of activities of daily living in health smart homes: sensors, algorithms, and first experimental results," IEEE Transaction on Information Technology in Biomedicine, vol. 14, no. 2, pp. 274-283, Mar. 2010. 
[44]Health Canada Division of Aging and Seniors, "Canada's Aging Population," 2002. http://dsppsd.pwgsc.gc.ca/Collection/H39-608-2002E.pdf.

[45]C. Rougier, J. Meunier, A. St-Arnaud, and J. Rousseau, "Monocular 3D head tracking to detect falls of elderly people," in Proc. $28^{\text {th }}$ International conference IEEE Engineering in Medicine and Biology Society, New York, NY, USA, 2006, pp. 6384-6387.

[46] http://www.who.int/topics/chronic_diseases/en/

[47] C.N. Scanaill, B. Ahearne, and G.M. Lyons, "Long-term telemonitoring of mobility trends of elderly people using SMS messaging," IEEE Transactions on Information Technology in Biomedicine, vol. 10, no.2, pp. 412-413, Apr. 2006.

[48] S.S. Birring, A.J. Ing, K. Chan, G. Cossa, S. Matos, M.D.L Morgan, and I.D. Pavord, "Obstructive sleep apnoea: a cause of chronic cough," Cough, vol. 3, 2007.

[49]P.V. Dicpinigaitis, G.L. Colice, M.J. Goolsby, G.I. Rogg, S.L. Spector, and B. Winther, "Acute cough: a diagnostic and therapeutic challenge," Cough, vol. 5, 2009.

[50]M. Emam, J.F Renaud de la Faverie, N. Gharbi, and M.I. El-Gohary, "Characterization of lung's emphysema distribution: numerical assessment of disease development," Proc. $4^{\text {th }}$ International conference on New Trends in Information Science and Service Science, Gyeongju, Korea, May. 2010, pp. $464-469$.

[51]M. Blount, V.M. Batra, A.N. Capella, M.R. Ebling, W.F. Jerome, S.M. Martin, M. Nidd, M.R. Niemi, and S.P. Wright, "Remote health-care monitoring using personal care connect," IBM System Journal on Computing and Processing (Hardware/Software), vol. 46, pp. 95-113, Apr. 2010.

[52]M.M. Eastburn, P.H. Katelaris, and A.B. Chang, "Defining the relationship between gastroesophageal reflux and cough: probabilities, possibilities and limitations," Cough, vol. 3, 2007.

[53] The Milken Institute, "An unhealthy America: The economic burden of chronic disease charting a new course to save lives and increase productivity and economic growth". October 2007. [Online] http://www.milkeninstitute.org/healthreform/pdf/AnUnhealthyAmericaExecSumm.pdf

[54]J.B. Oostenbrink, M.P.M.H. Rutten-van Molken, M.J. AI, J.A. Van Noord, W. Vincken, "One-year cost-effectiveness of tiotropium versus ipratropium to treat chronic obstructive pulmonary disease," European Respiratory Journal, vol. 23, pp. 241-249, Feb. 2004.

[55]S. Junnila, H. Kailanto, J. Merilahti, A. Vainio, A. Vehkaoja, M. Zakrzewski, and J. Hyttinen, "Wireless, multipurpose in-home health monitoring platform: two case trials," IEEE Transaction on Information Technology in Biomedicine, vol. 14, no. 2, pp. 447-455, Mar. 2010.

[56] http://www.who.int/mediacentre/factsheets/fs307/en/index.html 
[57]P. Rashidi, D.J. Cook, "Keeping the resident in the loop: adapting the smart home to the user," IEEE Transaction on Sysems, Man and Cybernetics, Part A: Systems and Humans, vol. 39, no. 5, pp. 949959, Sep. 2009.

[58]P. Pirila and A.R.A. Sovijarvi, "Objective assessment of cough," European Respiratory Journal, vol. 8, pp. 1949-1956, Nov. 1995.

[59] S. Hadjikoutis, C.M. Wiles, and R. Eccles, "Cough in motor neuron disease: a review of mechanisms," QJM: an International Journal of Medicine, vol. 92, pp. 487-494, Sep. 1999.

[60]K.F. Chung, "Assessment and measurement of coughs: the value of new tools," Pulmonary Pharmacology and Therapeutics, vol. 15, pp. 267-272, May 2002.

[61] W. Thorpe, M. Kurver, G. King, and C. Salome, "Acoustic analysis of cough," in Proc. the Seventh Australian and New Zealand on Intelligent Information Systems Conference, Perth, Western Australia, Nov. 2001, pp. 391-394.

[62] http://www.summitmedicalgroup.com/library/adult_health/vocal_cord_lesions/

[63]P.A. Kvale, P.A. Selecky, and U.B.S. Prakash, "Palliative care in lung cancer: ACCP evidence-based clinical practice guidelines ( $2^{\text {nd }}$ edition)," Chest, vol. 132, no. 3, Sep. 2007.

[64]A. Lay-Ekuakille, P. Vergallo, A. Trotta, A. Trabacca, M.De Rinaldis, F. Angelillo, and L. Petrara, "Ergospirometric signal processing for human breath and force measurements," in Proc. IEEE International Workshop on Medical Measurements and Applications MeMeA, Ottawa, Canada, May 2010, pp. 113-116.

[65]A. Arcelus, R. Goubran, H. Sveistrup, M. Bilodeau, and F. Knoefel, "Context-aware smart home monitoring through pressure measurement sequences," in Proc. IEEE International Workshop on Medical Measurements and Applications MeMeA, Ottawa, Canada, May 2010, pp. 32-37.

[66] M.Q. Jing, C.C. Wang, and L.H. Chen, "A real-time unusual voice detector based on nursing at home," in Proc. Machine Learning and Cybernetics, Baoding, Hebei China, Aug. 2009, vol. 4, pp. 2368-2374.

[67] M.J. Doherty, L.J. Wang, S. Donague, M.G. Pearson, P. Downs, S.A.T. Stoneman, J.E. Earis, "The acoustic properties of capsaicin-induced cough in healthy subjects", European Respiratory Journal, , vol. 10, pp. 202-207, Jan. 1997.

[68] P.M. Olia, P. Sestini, and M. Vagliasindi, "Acoustic parameters of voluntary cough in healthy nonsmoking subjects", Respirology, vol. 5, issue 3, pp.283-287, Sep 2000.

[69]H. Lamraoui, A. Bonvilain, G. Robin, P. Mozer, A. Moreau-Gaudry, P. Cinquin, P.Y. Gumery, and S.Basrour, "Rectus abdominis electromyography and mechanomyography comparison for the 
detection of cough," in Proc. IEEE International Conference on Engineering in Medicine and Biology Society, Buenos Aires, Argentina, Sep. 2010, pp. 6502-6505.

[70] Y.H. Hiew, J.A. Smith, J.E. Earis, B.M.G. Cheetham, and A.A. Woodcock, "DSP algorithm for cough identification and counting," in Proc. Acoustics, Speech, and Signal Processing, Minneapolis, MN, USA, vol. 4, Apr. 1993, pp. 3888-3891.

[71]C.W. Thorpe, L.J. Toop, and K.P. Dawson, "Towards a quantitative description of asthmatic cough sounds," European Respiratory Journal, vol. 5, pp. 685-692, Jun. 1992.

[72] http://www.sound-effect.com/

[73] http://www.freesound.org/

[74] J. Widdicombe, and G. Gontana, "Cough: what's in a name?," European Respiratory Journal, vol. 28, pp. 10-15, Jul.. 2006. 